\title{
WHAT IS THE TOTAL DEUTERIUM ABUNDANCE IN THE LOCAL GALACTIC DISK? ${ }^{1}$
}

\author{
Jeffrey L. Linsky², Bruce T. Draine ${ }^{3}$, H. W. Moos $^{4}$, Edward B. Jenkins ${ }^{5}$, Brian E. Wood ${ }^{2}$, \\ Cristina Oliveira ${ }^{4}$, William P. Blair ${ }^{4}$, Scott D. Friedman ${ }^{6}$, Cecile Gry ${ }^{12}$, David Knauth ${ }^{7}$, \\ Jeffrey W. Kruk ${ }^{4}$, Sylvestre Lacour ${ }^{13}$, Nicolas Lehner ${ }^{8}$, Seth Redfield ${ }^{9}$, J. Michael Shull ${ }^{10}$, \\ George Sonneborn ${ }^{11}$, Gerard M. Williger ${ }^{4}$
}

\begin{abstract}
Analyses of spectra obtained with the Far Ultraviolet Spectroscopic Explorer (FUSE) satellite, together with spectra from the Copernicus and IMAPS instruments, reveal an unexplained very wide range in the observed deuterium/hydrogen $(\mathrm{D} / \mathrm{H})$ ratios for interstellar gas in the Galactic disk beyond the Local Bubble. We argue that spatial variations in the depletion of deuterium onto dust grains can explain these local variations in the observed gas-phase $\mathrm{D} / \mathrm{H}$ ratios. We present a variable deuterium depletion model that naturally explains the constant measured values of $\mathrm{D} / \mathrm{H}$ inside the Local Bubble, the wide range of
\end{abstract}

\footnotetext{
${ }^{2}$ JILA, University of Colorado and National Institute of Standards and Technology, Boulder, CO 803090440; jlinsky@jila.colorado.edu.

${ }^{3}$ Department of Astrophysical Sciences, Princeton University, Princeton, NJ 08544-1001.

${ }^{4}$ Department of Physics and Astronomy, Johns Hopkins University, Baltimore, MD 21218.

${ }^{5}$ Princeton University Observatory, Princeton, NJ 08544.

${ }^{6}$ Space Telescope Science Institute, 3700 San Martin Drive, Baltimore, MD 21218.

${ }^{7}$ Department of Physics and Astronomy, Northwestern University, Evanston, IL 60208-2900.

${ }^{8}$ Department of Astronomy, University of Wisconson, 475 N. Charter St., Madison, WI 53706-1582.

${ }^{9}$ University of Texas at Austin, McDonald Observatory, Austin, TX 78712. Harlan J. Smith Postdoctoral Fellow.

${ }^{10}$ Center for Astrophysics and Space Astronomy, Dept. of Astrophysical and Planetary Sciences, University of Colorado, Boulder, CO 80309-0389.

${ }^{11}$ Laboratory for Observational Cosmology, NASA Goddard Space Flight Center, Code 665, Greenbelt, MD 20771.

${ }^{12}$ Laboratoire d'Astrophysique de Marseille, 13376 Marseille, France.

${ }^{13}$ Observatoire de Paris-Meudon, LESIA, 92195 Meudon, France.
} 
gas-phase $\mathrm{D} / \mathrm{H}$ ratios observed in the intermediate regime $(\log N(\mathrm{H} \mathrm{I})=19.2-$ 20.7), and the low gas-phase $\mathrm{D} / \mathrm{H}$ ratios observed at larger hydrogen column densities. We consider empirical tests of the deuterium depletion hypothesis: (i) correlations of gas-phase $\mathrm{D} / \mathrm{H}$ ratios with depletions of the refractory metals iron and silicon, and (ii) correlation with the $\mathrm{H}_{2}$ rotational temperature. Both of these tests are consistent with deuterium depletion from the gas phase in cold, not recently shocked, regions of the ISM, and high gas-phase D/H ratios in gas that has been shocked or otherwise heated recently. We argue that the most representative value for the total (gas plus dust) $\mathrm{D} / \mathrm{H}$ ratio within $1 \mathrm{kpc}$ of the Sun is $\geq 23.1 \pm 2.4(1 \sigma)$ parts per million (ppm). This ratio constrains Galactic chemical evolution models to have a very small deuterium astration factor, the ratio of primordial to total $(\mathrm{D} / \mathrm{H})$ ratio in the local region of the Galactic disk, which we estimate to be $f_{d} \leq 1.19_{-0.15}^{+0.16}(1 \sigma)$ or $\leq 1.12 \pm 0.14(1 \sigma)$ depending on the adopted light element nuclear reaction rates.

Subject headings: ISM: abundances — ISM: structure — Galaxy: abundances — Galaxy: solar neighborhood — Galaxy: deuterium — Ultraviolet: ISM

\section{INTRODUCTION}

Accurate measurements of the deuterium/hydrogen $(\mathrm{D} / \mathrm{H})$ ratio (by number) provide critically important tests for models of primordial nucleosynthesis, Galactic chemical evolution, and the chemical properties of the intergalactic medium (IGM). Among all of the light elements, deuterium arguably provides the most stringent tests of our understanding of these three critically important topics, because the evolution of deuterium is predicted to be rather simple and the dependence of $\mathrm{D} / \mathrm{H}$ varies steeply with baryonic density. Epstein, Lattimer, \& Schramm (1976) and others have argued that deuterium was formed only in the Big Bang and that D is easily converted to ${ }^{3} \mathrm{He},{ }^{4} \mathrm{He}$, and heavier elements by nuclear reactions in stars, a process commonly called astration. Thus, as stars evolve, the interstellar medium (ISM) receives deuterium-depleted and metal-enhanced material from stellar winds and supernova explosions, leading to a monotonic decrease in the $\mathrm{D} / \mathrm{H}$ ratio with increased processing through stars. Other light elements (e.g., ${ }^{3} \mathrm{He},{ }^{4} \mathrm{He},{ }^{7} \mathrm{Li}$ ) have more complex creation and destruction histories or otherwise provide less sensitive tests of these theories

\footnotetext{
${ }^{1}$ Based on observations made with the NASA-CNES-CSA Far Ultraviolet Spectroscopic Explorer. FUSE is operated for NASA by the Johns Hopkins University under NASA contract NAS5-32985.
} 
(cf. Boesgaard \& Steigman 1985; Wilson \& Rood 1994). Infall of less astrated gas, which is deuterium rich and metal poor, from the IGM and small galaxies captured by the Milky Way Galaxy, complicates models of Galactic chemical evolution, because the rate of infall, its time and spatial dependence, and the timescale for mixing with other gas are poorly known.

Although based on different physical processes and relating to very different times in the early universe, the two main approaches for determining the primordial $\mathrm{D} / \mathrm{H}$ ratio, $(\mathrm{D} / \mathrm{H})_{\text {prim }}$, are now in agreement. The first approach is to measure the column densities of D I and H I in quasar absorption line systems. Kirkman et al. (2003) have determined a mean value of $\mathrm{D} / \mathrm{H}=27.8_{-3.8}^{+4.4}$ parts per million $(\mathrm{ppm})$ by averaging the $\log (\mathrm{D} / \mathrm{H})$ values using column densities $N(\mathrm{H} \mathrm{I})$ and $N(\mathrm{D} \mathrm{I})$ obtained from absorption line measurements of gas in the lines of sight toward five quasars. Most of these measurements use high resolution spectra obtained with the Keck telescope. Since the observed gas has very low metallicity, these values of $\mathrm{D} / \mathrm{H}$ are likely close to, but perhaps slightly smaller than, the primordial (Big Bang) value, $(\mathrm{D} / \mathrm{H})_{\text {prim. }}$. The measurement of $\mathrm{D} / \mathrm{H}=22 \pm 7 \mathrm{ppm}$ for the high velocity cloud Complex C (Sembach et al. 2004) likely refers to gas located 6-10 kpc into the Galactic halo with low metallicity, 10-30\% solar abundance (Collins et al. 2003), and this value should be somewhat smaller than $(\mathrm{D} / \mathrm{H})_{\text {prim }}$.

The second approach involves the analysis of data from the Wilkinson Microwave Anisotropy Probe (WMAP) satellite and other cosmic microwave background experiments. Assuming a flat $\Lambda$-dominated universe and other standard assumptions, several authors (Spergel et al. 2003; Cyburt, Fields, \& Olive 2003; Coc et al. 2004; Sánchez et al. 2006) have derived tight constraints on the baryon closure parameter, $\Omega_{\mathrm{b}}$, and the Hubble constant. Together with other constraints from the power spectrum of galaxy clustering and $L \alpha$ forest data, they find that $\Omega_{b} h^{2}=0.0224$ with a $1 \sigma$ error of about $4 \%$. These results are summarized in Table 1 . The ratio of baryons to photons (multiplied by $10^{10}$ ), $\eta_{10}$, is simply related to $\Omega_{b} h^{2}$ (Burles, Nollett, \& Turner 2001). In the standard big bang nucleosynthesis (SBBN) model, $\eta_{10}$ is the single parameter that predicts the primordial light element abundances including $(\mathrm{D} / \mathrm{H})_{\text {prim. }}$. For example, Spergel et al. (2003), Coc et al. (2004), and Sánchez et al. $(2006)$ obtain $(\mathrm{D} / \mathrm{H})_{\text {prim }} \approx 26 \mathrm{ppm}$. Using the same values of $\Omega_{b} h^{2}$ and $\eta_{10}$ but a more recent compilation of nuclear reaction rates (Angulo et al. 1999), Cyburt, Fields, \& Olive $(2003)$ obtain $(\mathrm{D} / \mathrm{H})_{\text {prim }}=27.5_{-1.9}^{+2.4} \mathrm{ppm}$. The somewhat different values of $(\mathrm{D} / \mathrm{H})_{\text {prim }}$ arise from the different authors treating the light element nuclear reaction rates and error propagation somewhat differently (cf. Nollett \& Burles 2000). We therefore use in the subsequent analysis two different values for $(\mathrm{D} / \mathrm{H})_{\text {prim }}, 27.5_{-1.9}^{+2.4} \mathrm{ppm}$ (Cyburt, Fields, \& Olive 2003) and $26.0_{-1.7}^{+1.9} \mathrm{ppm}$ (Coc et al. 2004). Both values are consistent with the quasar absorption line result for $(\mathrm{D} / \mathrm{H})_{\text {prim }}$. 
Beginning with observations by the Copernicus satellite, data from high-resolution ultraviolet spectrometers on several satellites have been analyzed to measure $\mathrm{D} / \mathrm{H}$ in the Galactic ISM. These results provide insight into the abundance of deuterium in the present epoch, which with measurements of various metal abundances constrain models of Galactic chemical evolution. The International Ultraviolet Explorer (IUE) satellite and more recently the Goddard High Resolution Spectrometer (GHRS) and Space Telescope Imaging Spectrograph (STIS) instruments on the Hubble Space Telescope (HST) have provided UV spectra of the Ly $\alpha$ absorption lines of H I $\left(1215.67 \AA\right.$ ) and D I (shifted by $-82 \mathrm{~km} \mathrm{~s}^{-1}$ relative to the H I line), which are needed to measure D/H in the ISM along lines of sight to stars located within a few hundred parsecs of the Sun. The same analysis technique has been applied to hydrogen and deuterium lines higher in the Lyman series that lie in the spectral range 912$1025 \AA$, using data obtained with the Copernicus, the Interstellar Medium Absorption Profile Spectrograph (IMAPS), and the FUSE satellite. For a description of the FUSE satellite and its operations, see Moos et al. (2000) and Sahnow et al. (2000).

The far-UV spectral range is essential for sampling more distant lines of sight using hot subdwarfs and white dwarfs as background sources, because the decreasing opacity in the higher Lyman lines allows one to observe the deuterium lines without masking by the nearby very opaque broad hydrogen lines. For example, the dark core of the H I Ly $\alpha$ line absorption broadens to mask the D I Ly $\alpha$ line when the hydrogen column density $\log N(\mathrm{H} \mathrm{I})>18.7$, but at $\operatorname{Ly} \gamma(972 \AA)$ the horizon for detecting the DI Ly $\gamma$ line extends to $\log N(\mathrm{H} \mathrm{I}) \approx 20.0$. Measurements of $\mathrm{D} / \mathrm{H}$ using the higher Lyman series lines are feasible to about $\log N(\mathrm{H} \mathrm{I}) \approx 21$ (cf. Hoopes et al. 2003), where the density of overlapping $\mathrm{H}_{2}$ lines and the large widths of the interstellar H I Lyman lines determine the final horizon for such analyses.

There are several subtle effects that can enter the analysis of D I and H I lines in the far UV. One disadvantage is that, for long lines of sight, there is an increased chance of weak features at large velocity displacements. An $\mathrm{H}$ I feature near $-82 \mathrm{~km} \mathrm{~s}^{-1}$ can masquerade as D I absorption. An important advantage is that the far UV includes a large number of Lyman lines with a wide range of optical depths. Thus, measurements of $N(\mathrm{H} \mathrm{I})$ and $N(\mathrm{D}$ I) can be based on several spectral lines in the Lyman series. Another advantage is that, for the higher Lyman lines, the upper state lifetimes are long, decreasing the damping and better separating the H I and D I absorption for each line in the Lyman series and between adjacent lines in the series.

Since the very first of these measurements, a disturbing aspect of the $\mathrm{D} / \mathrm{H}$ studies has been the wide range of measured values with no apparent pattern in the data or a convincing explanation for it. We address the first part of this problem with the latest data, including 
many measurements with FUSE; we conclude ( we show that there is a consistent pattern. We propose that the variations arise from timedependent deuterium depletion onto dust grains (see $\S 4$ and $\S 5$ ), and we examine two critical tests (see $\S 6$ ) of this model. The first test ( 66.1$)$ uses iron and silicon depletions as proxies for grain destruction, and the second test (§6.2) uses correlations of $\mathrm{D} / \mathrm{H}$ with $\mathrm{H}_{2}$ excitation temperatures. We conclude (see $\S 7$ and $\S 9$ ) that the most likely value for the total $\mathrm{D} / \mathrm{H}$ ratio within $1 \mathrm{kpc}$ of the Sun is about $45 \%$ larger than the previously assumed value of $\approx 15$ ppm, which provides a revised constraint on models of Galactic chemical evolution. Also, the inferred depletion of deuterium from the gas phase places constraints on the physics and chemistry of interstellar dust. See Linsky (2006) for an early version of this paper.

\section{MEASUREMENTS OF D/H IN THE LOCAL GALACTIC DISK}

Using FUSE data, Wood et al. (2004) measured (D/H) gas along the lines of sight to two hot subdwarf stars, JL 9 and LS 1274, located at distances of $590 \pm 160$ pc and $580 \pm 100$ pc, respectively. They also summarized the $(\mathrm{D} / \mathrm{H})_{\text {gas }}$ measurements observed by the previously mentioned satellites for 38 lines of sight to stars located between $3.2 \mathrm{pc}$ and $2200 \mathrm{pc}$ from the Sun. These column densities are summarized in Table 2 together with the new results for the PG 0038+199 (Williger et al. 2005), HD 90087 (Hébrard et al. 2005), LSE 44 (Friedman et al. 2006), WD 1034+001 and TD1 32709 (Oliveira et al. 2006) lines of sight and (D/H) gas for Lan 23 (Oliveira et al. 2003), which was not included in previous compilations owing to the large uncertainty in $(\mathrm{D} / \mathrm{H})_{\text {gas }}$. Table 3 lists the $(\mathrm{D} / \mathrm{H})_{\text {gas }}$ ratios computed from the measured column densities in Table 2 , and the $1 \sigma$ errors in $(\mathrm{D} / \mathrm{H})_{\text {gas }}$ are computed from the square root of the sum of the squares of the percent errors in $N(\mathrm{D} \mathrm{I})$ and $N(\mathrm{H} \mathrm{I})$. When authors present their results for $(\mathrm{D} / \mathrm{H})_{\text {gas }}$ but not $N(\mathrm{D} \mathrm{I})$, we have computed $N(\mathrm{D} \mathrm{I})$ and its uncertainty to be consistent with the published values of $(\mathrm{D} / \mathrm{H})_{\text {gas }}$ and $N(\mathrm{H} \mathrm{I})$. The $(\mathrm{D} / \mathrm{H})_{\text {gas }}$ measurements plotted in Figure 1 show a complex picture that, as we shall see, seriously challenges previously held ideas concerning Galactic chemical evolution.

Table 2 lists the most recent measurements of $N(\mathrm{H} \mathrm{I})$ and $N(\mathrm{D} \mathrm{I})$, ordered by increasing $N(\mathrm{H} \mathrm{I})$, for interstellar gas along the lines of sight to the listed stars, obtained using the FUSE, Copernicus, IMAPS, and GHRS and STIS instruments on $H S T$. $(\mathrm{D} / \mathrm{H})_{\text {gas }}$, which is derived from the $N(\mathrm{D} \mathrm{I}) / N(\mathrm{H} \mathrm{I})$ ratio, is not always the same as originally reported because there are measurements of $N(\mathrm{H} \mathrm{I})$ obtained more recently with higher $\mathrm{S} / \mathrm{N}$ and/or spectral resolution than the corresponding $N(\mathrm{D} \mathrm{I})$ measurements. This is typically the case when the only measurement of $N(\mathrm{D} \mathrm{I})$ was obtained with the Copernicus satellite and $N(\mathrm{H} \mathrm{I})$ was later measured from $H S T$ spectra. For the sightlines to $\mu \mathrm{Col}, \beta \mathrm{CMa}, \theta \mathrm{Car}$, and $\lambda$ Sco, we 
list $N(\mathrm{H} \mathrm{I})$ and $N(\mathrm{D} \mathrm{I})$ for a specified velocity component (see notes to Table 2) rather than for the entire inhomogeneous line of sight, since the high quality spectra enabled the authors to identify the velocity components and measure the column densities in each component, although the components were likely not resolved.

For the sightlines that extend through the Local Bubble to more distant stars, we also list $(\mathrm{D} / \mathrm{H})_{\text {gas }-\mathrm{LB}}$, the value of $(\mathrm{D} / \mathrm{H})_{\text {gas }}$ for the line of sight beyond the Local Bubble foreground, obtained by subtracting $N(\mathrm{H} \mathrm{I})_{\mathrm{LB}}=10^{19.20} \mathrm{~cm}^{-2}$ and $N(\mathrm{D} \mathrm{I})_{\mathrm{LB}}=10^{14.39} \mathrm{~cm}^{-2}$ from the measured values of $N(\mathrm{H} \mathrm{I})$ and $N(\mathrm{D}$ I) for the full line of sight. We assume that the Local Bubble extends to $\log N(\mathrm{H} \mathrm{I})=19.2$, since beyond that point the $(\mathrm{D} / \mathrm{H})_{\text {gas }}$ values are no longer consistent with a constant value. Sfeir et al. (1999) place the edge of the Local Bubble at $\log N(\mathrm{H} \mathrm{I}) \sim 19.3$, which corresponds to a $20 \mathrm{~m} \AA$ equivalent width of the Na I $5892 \AA$ line formed in cold interstellar gas. Within the Local Bubble, we adopt $(\mathrm{D} / \mathrm{H})_{\text {gas }}=15.6 \pm 0.4 \mathrm{ppm}$ (Wood et al. 2004). Given the complex three dimensional shape of the Local Bubble (Lallement et al. 2003), these assumptions for its column densities are approximations, but they provide good first estimates of the foreground required for isolating the deuterium and hydrogen column densities of the gas lying beyond the Local Bubble. This is especially important for lines of sight that do not extend very far beyond the Local Bubble, but this correction is small for the more distant lines of sight because the Local Bubble contribution to the total is minor. Those lines of sight that are definitely inside the Local Bubble are flagged as LBg in the eighth column of Table 2.

Figure 1 shows the $(\mathrm{D} / \mathrm{H})_{\text {gas }}$ ratios for the 47 lines of sight as functions of hydrogen column density. The $(\mathrm{D} / \mathrm{H})_{\text {gas }}$ ratios extend over an unexpectedly wide range, but Wood et al. (2004) proposed that these results naturally fit into three regimes. For $\log N(\mathrm{H} \mathrm{I})<19.2$ (in $\mathrm{cm}^{-2}$ units), corresponding to sightlines extending to the edge of the Local Bubble near $100 \mathrm{pc},(\mathrm{D} / \mathrm{H})_{\text {gas }}$ has a constant value of $15.6 \pm 0.4 \mathrm{ppm}$, where the quoted uncertainty is the $1 \sigma$ error in the mean for the 23 lines of sight within this regime. This constant value for the local $(\mathrm{D} / \mathrm{H})_{\text {gas }}$ ratio had been previously established based on HST data (Linsky 1998, 2003), but the addition of new lines of sight observed by FUSE (Moos et al. 2002) strongly supports this result. The very different values of $(\mathrm{D} / \mathrm{H})_{\text {gas }}$ measured in the distant $(\log N(\mathrm{H} \mathrm{I})>20.7)$ and intermediate $(\log N(\mathrm{H} \mathrm{I})=19.2-20.7)$ regimes beyond the Local Bubble are described at the end of this section.

Hébrard \& Moos (2003), Hébrard et al. (2005) and others have derived $(\mathrm{D} / \mathrm{H})_{\text {gas }}$ in an alternative way from the product $(\mathrm{D} / \mathrm{O})_{\text {gas }} \times(\mathrm{O} / \mathrm{H})_{\text {gas }}$. Since the charge-exchange reaction between $\mathrm{O} \mathrm{I}$ and $\mathrm{H} \mathrm{I}$ is fast, the ionization of oxygen, hydrogen, and deuterium are very nearly the same. In the ionization equilibrium calculations for their best models (Models 2 and 8) for the Local Interstellar Cloud, Frisch \& Slavin (2003) find that 
each element is about $30 \%$ ionized. Thus in the gas phase $(\mathrm{O} / \mathrm{H})=N(\mathrm{O} \mathrm{I}) / N(\mathrm{H} \mathrm{I})=$ $[N(\mathrm{O} \mathrm{I})+N(\mathrm{O} \mathrm{II})] /[N(\mathrm{H} \mathrm{I})+N(\mathrm{H} \mathrm{II}]$, and a similar relation can be written for $(\mathrm{D} / \mathrm{O})$. Empirically, the abundance of $\mathrm{O}$ relative to $\mathrm{H}$ shows little evidence for spatial variation (Meyer et al. 1998; André et al. 2003; Cartledge et al. 2004). Also, the outcomes for (D/O)gas may not suffer from the effects of line saturation, as there are O I lines that are optically thin. All three of these considerations should make O I a good proxy for H I. The (D/O) gas ratio should therefore behave in a very similar way to $(\mathrm{D} / \mathrm{H})_{\text {gas }}$. The problems with this technique are that uncertainties in two different measurements must be considered instead of one, and the amount of depletion of oxygen from the gas phase may depend on density (Cartledge et al. 2004). Table 4 summarizes the recent measurements of $(\mathrm{O} / \mathrm{H})_{\text {gas }}$. Oliveira et al. (2005) found that inside the Local Bubble, $(\mathrm{O} / \mathrm{H})_{\text {gas }}$ is constant with a mean value of $345 \pm 19$ ppm. Meyer et al. (1998), Meyer (2001), and André et al. (2003) find slightly higher values of $(\mathrm{O} / \mathrm{H})_{\text {gas }}$ for lines of sight extending beyond the Local Bubble with perhaps a slight decrease in $(\mathrm{O} / \mathrm{H})_{\text {gas }}$ at large column densities $(\log \mathrm{N}(\mathrm{H} \mathrm{I})>21.5)$. We assume $(\mathrm{D} / \mathrm{O})_{\text {gas }}=(3.84 \pm 0.16) \times 10^{-2}(1 \sigma)$, the mean value that Hébrard \& Moos (2003) find for lines of sight inside the Local Bubble, and compute the values of $(\mathrm{D} / \mathrm{H})_{\text {gas }}$ listed in Table 4. Inside the Local Bubble, using the value of $(\mathrm{O} / \mathrm{H})_{\text {gas }}$ obtained by Oliveira et al. (2005), we compute $(\mathrm{D} / \mathrm{H})_{\text {gas }}=13.2 \pm 0.9 \mathrm{ppm}$. For values of $(\mathrm{O} / \mathrm{H})_{\text {gas }}$ derived from longer lines of sight (André et al. 2003), we find $(\mathrm{D} / \mathrm{H})_{\text {gas }}=13.9 \pm 0.7$ to $15.7 \pm 0.9 \mathrm{ppm}$, depending on the number of lines of sight included. In their Models 2 and 8, Frisch \& Slavin (2003) conclude that $(\mathrm{O} / \mathrm{H})_{\text {gas }}=380 \pm 30^{2} \mathrm{ppm}$, leading to $(\mathrm{D} / \mathrm{H})_{\text {gas }}=14.6 \pm 1.3 \mathrm{ppm}$. These alternative ways of determining $(\mathrm{D} / \mathrm{H})_{\text {gas }}$ are in agreement or smaller by $1-2 \sigma$ compared with the direct measurement of $(\mathrm{D} / \mathrm{H})_{\text {gas }}=15.6 \pm 0.4 \mathrm{ppm}$ obtained by Wood et al. (2004).

To put these numbers into perspective, we note the recent measurements of the solar ratio, $(\mathrm{O} / \mathrm{H})_{\odot}=458 \pm 53 \mathrm{ppm}$ (Asplund et al. 2004) and $390 \pm 63 \mathrm{ppm}$ (Meléndez 2004). Bahcall, Serenelli, \& Basu (2005) argue that the Asplund et al. (2004) value for the solar oxygen abundance is too low to explain the helioseismological determinations of convection zone parameters and surface helium composition, but a recent increase in the estimated opacity near the base of the solar convective zone (Seaton \& Badnell 2004) minimizes the problem even for the Meléndez (2004) low abundance. In $\S 3$ we argue that about 120 ppm of oxygen could be depleted on to grains if $\mathrm{Mg}, \mathrm{Fe}$, and Si have solar abundances in the ISM, but Frisch \& Slavin (2003) conclude that the ISM abundances of these elements may be only 60-70\% solar. We therefore take the measured solar value (458 $\pm 53 \mathrm{ppm})$ and that value less $120 \mathrm{ppm}$ as a plausible range in $(\mathrm{O} / \mathrm{H})_{\text {gas }}$. Then the plausible range in the inferred $(\mathrm{D} / \mathrm{H})_{\text {gas }}$ $=(\mathrm{D} / \mathrm{O})_{\text {gas }} \times(\mathrm{O} / \mathrm{H})$ is $13.0 \pm 2.0 \mathrm{ppm}$ to $17.6 \pm 2.1 \mathrm{ppm}$. All of the Local Bubble values

\footnotetext{
${ }^{2}$ We arbitrarily assign an error of $8 \%$ to their value of $(\mathrm{O} / \mathrm{H})_{\text {gas }}$ based on the discussion in their paper.
} 
of $(\mathrm{D} / \mathrm{H})_{\text {gas }}$ measured directly and indirectly (see Table 4) fall well within this range. The differences among these values and with the directly measured $(\mathrm{D} / \mathrm{H})_{\text {gas }}=15.6 \pm 0.4 \mathrm{ppm}$ may represent measurement uncertainty and perhaps some systematic effects.

An important new result noted for $(\mathrm{D} / \mathrm{H})_{\text {gas }}$ measurements by Hébrard \& Moos (2003) and emphasized by Wood et al. (2004) is that sightlines with large $N(\mathrm{H} \mathrm{I})$ appear to have systematically low values of $(\mathrm{D} / \mathrm{H})_{\text {gas }}$. Wood et al. (2004) called attention to the clump of four low $(\mathrm{D} / \mathrm{H})_{\text {gas }}$ values at $\log N(\mathrm{H} \mathrm{I})>20.7$, which we refer to as the distant regime. Whereas unambiguously low values for $\mathrm{D} / \mathrm{H}$ in lines of sight extending beyond the Local Bubble have been known since the Copernicus results for $\delta$ Ori, $\epsilon$ Ori, and $\theta$ Car (Jenkins et al. 1999; Laurent, Vidal-Madjar, \& York 1979; Allen, Jenkins, \& Snow 1992), the new results for JL 9 and LSS 1274, together with the $(\mathrm{D} / \mathrm{H})_{\text {gas }}$ values determined from FUSE observations of HD 195965 (794 \pm 200 pc) and HD 191877 (2200 \pm 550 pc) from Hoopes et al. (2003) and HD 90087 (2740 \pm 800 pc) from Hébrard et al. (2005) indicate an emerging pattern. For these five long lines of sight, $(\mathrm{D} / \mathrm{H})_{\text {gas }}=8.6 \pm 0.8$ (standard deviation of the mean) ppm, nearly a factor of two below the Local Bubble value.

The intermediate regime for $\log N(H I)=19.2-20.7$ exhibits a factor of 4-5 range in $(\mathrm{D} / \mathrm{H})_{\text {gas }}$ : lines of sight to five stars have high values (near $\left.22 \mathrm{ppm}\right)$, five stars have low values (near $6 \mathrm{ppm}$ ), and nine stars have intermediate values. Since (i) the Copernicus, IMAPS and FUSE data all show this wide spread in $(\mathrm{D} / \mathrm{H})_{\text {gas }}$ values in the intermediate regime, (ii) there does not appear to be any correlation of $(\mathrm{D} / \mathrm{H})_{\text {gas }}$ with Galactic latitude, longitude, or distance, and (iii) the $(\mathrm{D} / \mathrm{O})_{\text {gas }}$ values also show a large spread, we conclude that the wide spread in $(\mathrm{D} / \mathrm{H})_{\text {gas }}$ in the intermediate regime is not due to instrumental artifacts or unknown systematic errors, but is rather a robust result that requires a sensible scientific explanation. We call attention to the five high data points in the intermediate regime with $(\mathrm{D} / \mathrm{H})_{\text {gas }} \approx 22$ that will play an important role in our deuterium depletion model (see $\S 5$ ).

\section{PREVIOUS EXPLANATIONS OF THE D/H DATA}

The constant value of $(\mathrm{D} / \mathrm{H})_{\text {gas }}$ inside the Local Bubble suggests that this matter has experienced a similar history of nuclear processing, mixing, and deuterium depletion (cf. $\S 5)$. This history consists of star formation in the Scorpio-Centaurus Association some $10 \mathrm{Myr}$ ago and the creation of the Local Bubble by supernovae in the Lower Centaurus Crux subgroup of the Scorpio-Centaurus Association (Maíz-Apellániz 2001; Berghöfer \& Breitschwerdt 2002). Outside the Local Bubble, interstellar gas could have experienced different chemical evolution resulting from different rates of astration, infall of nearly primordial material from the Galactic halo or the IGM, and insufficient mixing to homogenize 
the Galactic ISM. However, Galactic chemical evolution models are faced with the severe challenge of explaining the factor of $4-5$ range in $(\mathrm{D} / \mathrm{H})_{\text {gas }}$ ratios just outside of the Local Bubble and the clumping of low $(\mathrm{D} / \mathrm{H})_{\text {gas }}$ values for the lines of sight with the largest values of $N(\mathrm{H} \mathrm{I})$. Recent Galactic chemical evolution models constructed to fit a wide range of constraints, including the abundances of deuterium, ${ }^{3} \mathrm{He},{ }^{4} \mathrm{He}, \mathrm{Li}$, and heavier elements created by astration, generally predict a moderate astration factor for the total amount of $(\mathrm{D} / \mathrm{H})_{\text {total }}$ in all forms, $\mathrm{f}_{d}=(\mathrm{D} / \mathrm{H})_{\text {prim }} /(\mathrm{D} / \mathrm{H})_{\text {total }} \approx 1.5$ (cf. Chiappini, Renda, \& Matteucci 2002; Romano et al. 2003). If we assume $(\mathrm{D} / \mathrm{H})_{\mathrm{prim}}=27.5 \mathrm{ppm}$, then the astration factors in the intermediate regime mentioned in the previous section would lie in the range of 1.25 to 4.6. How could such a large range in astration factors occur over distance scales of only 100-500 pc, but an apparently constant astration factor of 3.2 appear to be representative for longer distance scales?

Galactic chemical evolution models generally include enrichment of gas in the Galactic disk by the infall of less-astrated material from the IGM or gas from neighboring small galaxies. For example, Geiss, Gloeckler, \& Charbonnel (2002) find that the Local Bubble value of $(\mathrm{D} / \mathrm{H})_{\text {gas }}$ could be explained by a mixture of $60 \%$ gas representative of the protosolar nebula (with additional chemical processing over the last $4.6 \mathrm{Gyr}$ ) and $40 \%$ infalling gas with high $(\mathrm{D} / \mathrm{H})_{\text {gas }}$ and low metals representative of the Large Magellanic Cloud. While this mechanism may explain the Local Bubble abundances, variations of the percentages to explain the wide range of observed $(\mathrm{D} / \mathrm{H})_{\text {gas }}$ values would require that the infalling gas be spatially concentrated into small regions of the Galactic disk and then not be mixed. We note here that typical sizes for structures in the nearby infalling high-velocity cloud Complex C are about 100 pc (Collins, Shull, \& Giroux 2003). We discuss the infall hypothesis further in $\oint 8$.

Both spatially variable astration and infall models may, in principle, explain the $(\mathrm{D} / \mathrm{H})_{\text {gas }}$ measurements, but they require extreme assumptions concerning the deuterium destruction and infall rates to explain the observed $(\mathrm{D} / \mathrm{H})_{\text {gas }}$ ratios. Simulations with their threedimensional supernova-driven ISM model led de Avillez \& Mac Low (2002) to conclude that for the Galactic supernova rate, it takes some $350 \mathrm{Myr}$ to completely mix the ISM on distance scales from parsecs to kiloparsecs. In view of this relatively rapid mixing rate, we expect the total interstellar $\mathrm{D} / \mathrm{H}$ ratio in all forms, $(\mathrm{D} / \mathrm{H})_{\text {total }}$, to show little variation on length scales less than $1 \mathrm{kpc}$, unless one invokes localized starbursts sufficiently strong for astration to produce significantly lower $(\mathrm{D} / \mathrm{H})_{\text {total }}$ on a timescale shorter than $350 \mathrm{Myr}$. Both the variable astration and infall models fail to explain the absence of any correlation between the observed $(\mathrm{D} / \mathrm{H})_{\text {gas }}$ and $(\mathrm{O} / \mathrm{H})_{\text {gas }}$ ratios (e.g., Draine 2005), which should show a negative correlation for both models. We explore instead another physical process that could have a much larger effect on local values of the $(\mathrm{D} / \mathrm{H})_{\text {gas }}$ ratio than either astration or infall. 
Figure 2 shows observed values of $(\mathrm{D} / \mathrm{H})_{\text {gas }}$ and $(\mathrm{O} / \mathrm{H})_{\text {gas }}$ for all sightlines that have published column densities of $\mathrm{H}, \mathrm{D}$, and $\mathrm{O}$ with $1 \sigma$ uncertainties of less than 0.1 dex for each. The error bars in the figure are $1 \sigma$; because $N\left(\mathrm{H} \mathrm{I}+2 \mathrm{H}_{2}\right)$ appears in the denominator of both abscissa and ordinate, errors in $(\mathrm{O} / \mathrm{H})$ and $(\mathrm{D} / \mathrm{H})$ are partially correlated. Variations in astration or infall would produce anticorrelation of $(\mathrm{D} / \mathrm{H})_{\text {total }}$ and $(\mathrm{O} / \mathrm{H})_{\text {total }}$. Figure 2 shows the expected track of $(\mathrm{D} / \mathrm{H})_{\text {total }}$ vs. $(\mathrm{O} / \mathrm{H})_{\text {gas }}$ for chemical evolution model C-I of Chiappini et al. (2002), where (i) we have taken Chiappini et al.'s variation with galactocentric radius as a proxy for variations in astration and infall, (ii) we have rescaled the predicted $(\mathrm{D} / \mathrm{H})_{\text {total }}$ values slightly to be consistent with primordial $(\mathrm{D} / \mathrm{H})_{\text {total }} \approx 27.5 \mathrm{ppm}$ estimated from quasar absorption lines and $W M A P$, and (iii) we assume partial depletion of $\mathrm{O}$ into grains (see below), with $(\mathrm{O} / \mathrm{H})_{\text {gas }}=0.75(\mathrm{O} / \mathrm{H})_{\text {total }}$. The observed $(\mathrm{D} / \mathrm{H})_{\text {gas }}$ values fall systematically below the $(\mathrm{D} / \mathrm{H})_{\text {total }}$ values expected for at least this astration/infall model. The full (factor of $\sim 3)$ range of variation in $(\mathrm{D} / \mathrm{H})_{\text {gas }}$ is seen among the 6 sightlines with $(\mathrm{O} / \mathrm{H})_{\text {gas }}$ between 300 and $390 \mathrm{ppm}$. Variable astration and infall may account for a fraction of the variation in $(\mathrm{D} / \mathrm{H})_{\text {gas }}$, but it appears that most of the variation must be due to some other mechanism - such as depletion of D onto dust grains.

The median value of $(\mathrm{O} / \mathrm{H})_{\text {gas }}$ in Figure 2 is $363 \mathrm{ppm}$, but it is likely that another $\sim 120 \mathrm{ppm}$ is locked up in grains in silicates or oxides (assuming the grains contain $\sim 90 \%$ of solar abundances of $\mathrm{Mg}, \mathrm{Fe}$, and $\mathrm{Si}$, with composition $\left.\mathrm{Mg}_{x} \mathrm{Fe}_{2-x} \mathrm{SiO}_{4}\right){ }^{3}$ From these wellcharacterized sightlines, we therefore estimate $(\mathrm{O} / \mathrm{H})_{\text {total }} \approx 483 \mathrm{ppm}$ in the local ISM, slightly higher than but consistent with the recent estimate for the solar abundance $(\mathrm{O} / \mathrm{H})_{\odot}=$ $458 \pm 53 \mathrm{ppm}$ (Asplund et al. 2004). Thus we estimate that $\mathrm{O}_{\text {gas }} / \mathrm{O}_{\text {total }} \approx(363 / 483)=0.75$ for diffuse regions. Note that for a primordial $\mathrm{D} / \mathrm{H}=27 \mathrm{ppm}$ and $(\mathrm{O} / \mathrm{H})_{\text {gas }} /(\mathrm{O} / \mathrm{H})_{\text {total }} \approx 0.75$, the Chiappini et al. astration + infall model $\mathrm{C}-\mathrm{I}$ (see Figure 2 ) has $(\mathrm{D} / \mathrm{H})_{\text {total }}$ reduced to $23 \mathrm{ppm}$ by the time $(\mathrm{O} / \mathrm{H})_{\text {gas }}$ has risen to $\approx 360 \mathrm{ppm}$, very close to the measured value of $345 \pm 19$ ppm (Oliveira et al. 2005) for the solar neighborhood. We will return to this point in $\S 8$.

\section{DEUTERIUM DEPLETION ONTO DUST GRAINS}

It is important to recognize that all of the direct $(\mathrm{D} / \mathrm{H})_{\text {gas }}$ measurements refer to interstellar gas, whereas the Galactic chemical evolution and primordial nucleosynthesis models refer to $(\mathrm{D} / \mathrm{H})$ in all forms, $(\mathrm{D} / \mathrm{H})_{\text {total }}$. Dust grains exist in both the cold $\left(10-10^{2} \mathrm{~K}\right)$ and warm $\left(10^{3}-10^{4} \mathrm{~K}\right)$ phases of the ISM. In diffuse interstellar clouds, $50-90 \%$ of elements

\footnotetext{
${ }^{3}$ The abundance of $\mathrm{H}_{2} \mathrm{O}$ is negligible outside of dark clouds.
} 
such as Mg, Si, and Fe reside in grains (e.g., Sembach \& Savage 1996; Jenkins 2004), and gas-phase abundances of such elements relative to hydrogen are much lower than the total elemental abundances. A similar process may preferentially deplete deuterium in the gas phase relative to hydrogen. This could lead to large spatial variations in the gas-phase ratio, $(\mathrm{D} / \mathrm{H})_{\text {gas }}$, while the total deuterium abundance, $(\mathrm{D} / \mathrm{H})_{\text {total }}$, including both gas and dust, remains roughly constant. If deuterium depletion onto dust grains is important, then the total Galactic D/H ratio cannot be extracted from gas-phase measurements that ignore deuterium locked in dust grains.

Jura (1982) first proposed that the depletion of deuterium onto dust grains might explain the factor of two difference in $(\mathrm{D} / \mathrm{H})_{\text {gas }}$ values for the lines of sight to $\zeta$ Pup and $\delta$ Ori obtained from the analysis of Copernicus spectra (Vidal-Madjar et al. 1977; Laurent, Vidal-Madjar, \& York 1979). Jura argued that the sticking probability of deuterium onto dust grains should be high and that deuterium should be more tightly bound to the dust than hydrogen, since the zero-point energies of deuterium-metal bonds are lower than for the corresponding hydrogen-metal bonds. Numerical calculations of the chemistry of grain mantles by Tielens (1983) showed that deuterium can be highly enriched in grains, because the lower zeropoint energy for deuterium bonds as compared to corresponding hydrogen bonds strongly favors the formation of deuterated molecules like HDCO on grain surfaces (cf. Turner 1990), effectively removing deuterium from the gas phase in dark regions where grains are coated with ices.

Draine (2004, 2006) developed this idea further by noting that the C-D bond energy in polycyclic aromatic hydrocarbon (PAH) molecules is $0.083 \mathrm{eV}$ larger than the $\mathrm{C}-\mathrm{H}$ bond. If $\mathrm{H}$ and $\mathrm{D}$ in the gas were in thermodynamic equilibrium with deuterated $\mathrm{PAH}$ material, one would have $(\mathrm{D} / \mathrm{H})_{\text {dust }} /(\mathrm{D} / \mathrm{H})_{\text {gas }} \approx e^{970 K / T_{\text {dust }}}$, which exceeds $5 \times 10^{4}$ for $T_{\text {dust }}<90 \mathrm{~K}$. Although thermodynamic equilibrium arguments must be regarded with suspicion in the nonequilibrium ISM, Draine argued that it is plausible to consider that in a steady-state the grains might have $(\mathrm{D} / \mathrm{H})_{\text {dust }} /(\mathrm{D} / \mathrm{H})_{\text {gas }}$ as high as $5 \times 10^{4}$ or more.

Contemporary grain models require a substantial amount of hydrogen-bearing carbonaceous material. From the relative strengths and profiles of different $\mathrm{C}-\mathrm{H}$ stretching modes, Pendleton \& Allamandola (2002) estimate that $\sim 85 \%$ of the carbon in dust is in aromatic form, with $\mathrm{H} / \mathrm{C} \approx 0.35$, and $\sim 15 \%$ of the carbon is aliphatic, with $\mathrm{H} / \mathrm{C} \approx 2.1$. If ( $\mathrm{C}$ in grains $) /(\mathrm{H}$ total $) \approx 200 \mathrm{ppm}$, as estimated by Draine $(2004)$, then the aromatic hydrocarbon material would contain $(\mathrm{H}$ in aromatics $) /(\mathrm{H}$ total $) \approx 60 \mathrm{ppm}$. Suppose that in the local Galactic disk $(\mathrm{D} / \mathrm{H})_{\text {total }}=22 \mathrm{ppm}$, and that in the aromatic material the $\mathrm{D} / \mathrm{H}$ ratio is enhanced so that $(\mathrm{D} / \mathrm{H})_{\text {dust }}=0.27$. Then $(\mathrm{D} / \mathrm{H})_{\text {gas }}=(\mathrm{D} / \mathrm{H})_{\text {total }}-(\mathrm{D} / \mathrm{H})_{\text {dust }} \times$ $(\mathrm{H})_{\text {dust }} / \mathrm{H}_{\text {total }} \approx 6 \mathrm{ppm}$. Therefore we see that if $\mathrm{D}$ enrichment can bring the aromatic grains 
to $(\mathrm{D} / \mathrm{H})_{\text {dust }} \approx 0.27$, the gas phase deuterium abundance could be reduced to $\sim 6 \mathrm{ppm}$, which would be sufficient to explain the lowest values of $(\mathrm{D} / \mathrm{H})_{\text {gas }}$ observed. This would require extreme enrichment of the grains, relative to the gas, by a factor $(\mathrm{D} / \mathrm{H})_{\text {dust }} /(\mathrm{D} / \mathrm{H})_{\text {gas }} \approx$ $0.27 / 6 \mathrm{ppm} \approx 5 \times 10^{4}$. This is possible for $T_{\text {dust }}<90 \mathrm{~K}$ (see above). If the aliphatic and aromatic hydrocarbons are equally D-enriched, then $(\mathrm{D} / \mathrm{H})_{\text {dust }} /(\mathrm{D} / \mathrm{H})_{\text {gas }} \approx 2 \times 10^{4}$ would suffice to explain the sightlines with the lowest values of $(\mathrm{D} / \mathrm{H})_{\text {gas }} \approx 6 \mathrm{ppm}$, with $(\mathrm{D} / \mathrm{H})_{\mathrm{dust}} \approx 0.13$.

Figure 1 shows that $(\mathrm{D} / \mathrm{H})_{\text {gas }}$ varies from one sightline to another, covering a range from $\sim 6$ ppm to $\sim 22$ ppm. Draine $(2004,2006)$ and Wood et al. (2004) suggest a dynamic model of the ISM in which deuterium is depleted from the gas phase onto dust grains over time until the D-bearing grains are eroded or destroyed by sputtering or grain-grain collisions in strong shocks from supernova remnants, or by strong UV radiation fields from nearby hot stars, thereby returning the sequestered deuterium back to the gas phase. Is this idea supported by observations, and can it explain the three regime interpretation for the $(\mathrm{D} / \mathrm{H})_{\text {gas }}$ data shown in Fig. 1?

The most direct test of the deuterium depletion model would be to measure $(\mathrm{D} / \mathrm{H})_{\text {dust }}$ in interstellar dust grains. Keller, Messenger, \& Bradley (2000) have performed this critical experiment by measuring the deuterium abundance in interplanetary dust particles containing amorphous carbonaceous material. They argue that sample L2009*E2, which was captured in the upper atmosphere of the Earth, originated in the ISM because of the very high D/H ratios measured for various molecules (which indicates formation at low temperatures where fractionation is important), the elevated ${ }^{15} \mathrm{~N} /{ }^{14} \mathrm{~N}$ ratio, and the particle size distribution. For the whole dust grain sample, they find that $(\mathrm{D} / \mathrm{H})_{\text {dust }}=220 \mathrm{ppm}$, but for one fragment of the sample $(\mathrm{D} / \mathrm{H})_{\text {dust }}=1900 \mathrm{ppm}$. If we assume that the local ISM value of $(\mathrm{D} / \mathrm{H})_{\text {gas }}$ is $15 \mathrm{ppm}$, then the $(\mathrm{D} / \mathrm{H})_{\text {dust }}$ enhancement factors are 15 for the entire sample and 130 for the fragment. This measurement alone provides a proof of concept that deuterium depletion onto grains does occur in the ISM, although the highest D/H ratio measured by Keller, Messenger, \& Bradley (2000) in this particle sample falls a factor of $\sim 70$ short of the factor of $\sim 10^{4}$ that is required for grain deuteration to explain the typical observed variations in $(\mathrm{D} / \mathrm{H})_{\text {gas. }}$ It is interesting that high values of $(\mathrm{D} / \mathrm{H})_{\text {dust }}$ are only detected in amorphous organic (i.e., carbonaceous) material in extraterrestrial samples (Keller, Messenger, \& Bradley 2000; Peeters et al. 2004).

As part of their paper summarizing the observational evidence for deuterated PAHs (hereafter called PADs) in the ISM, Peeters et al. (2004) point out that deuterium fractionations (the ratio of deuterated molecules to undeuterated molecules) for simple molecular species are typically 0.01 to 0.1 (e.g., Markwick, Charnley \& Millar 2001) and higher deuterium fractionations have been measured (e.g., Parise et al. 2002). Even triply deuterated 
ammonia (van der Tak et al. 2002) and methanol (Parise et al. 2004) have even been observed in cold interstellar clouds. Peeters et al. (2004) then argue that PADs are more stable, more abundant in the ISM compared to many of the simpler molecules that show deuterium enrichments, and carry more $\mathrm{H}$ atoms per molecule. PADs may, therefore, represent a large reservoir of deuterium-enriched species.

Peeters et al. (2004) then call attention to infrared emission features at $4.4 \mu \mathrm{m}$ and $4.65 \mu \mathrm{m}$ in ISO spectra of the Orion Bar and M17 and identify these features as C-D stretching modes in PADs. From the relative strengths of the emission bands of PADs and PAHs, Peeters et al. (2004) estimate that the $\mathrm{D} / \mathrm{H}$ ratio in these molecules is $0.17 \pm 0.03$ for the Orion Bar and $0.36 \pm 0.08$ for M17. These ratios are about $10^{4}$ times larger than for atomic deuterium and hydrogen in the gas phase, $(\mathrm{D} / \mathrm{H})_{\text {gas }}$. It is striking that the $\mathrm{D} / \mathrm{H}$ ratios required to account for the observed $4.4 \mu \mathrm{m}$ and $4.65 \mu \mathrm{m}$ emission are comparable to the degree of deuteration of PAH material argued for by Draine (2004, 2006), and which imply substantial depletion of $\mathrm{D}$ from the gas if this $\mathrm{D} / \mathrm{H}$ ratio were characteristic of all of the hydrocarbon material in grains. Thus, deuteration of PAHs over time in a cold environment could be an important process for removing deuterium from the gas phase, and Peeters et al. (2004) describe four different astrochemical processes by which this could occur.

Grains must be cold for the deuterium depletion process to operate on their surfaces. Even in the $7000 \mathrm{~K}$ gas in warm interstellar clouds inside the Local Bubble, the grains are cold because at the densities of diffuse clouds, grain heating is by absorption of UV photons from stars rather than by collisions with the gas; the grains cool by the emission of infrared radiation. The observed IR emission spectrum for wavelengths greater than 80 microns requires that typical grain temperatures are $\sim 18 \mathrm{~K}$ (Draine 2003).

Because $(\mathrm{D} / \mathrm{H})_{\text {gas }}$ varies from one sightline to another, $\mathrm{D}$ depletion and return of $\mathrm{D}$ to the gas must be dynamic processes. What is the timescale for D depletion? Draine (2006) considers reaction of $\mathrm{D}^{+}$ions with neutral PAHs, and reaction of $\mathrm{D}$ atoms with $\mathrm{PAH}^{+}$ ions, arguing that the impinging $\mathrm{D}$ will become chemically bound in the PAH, displacing a previously-bound $\mathrm{H}$ if necessary. Considering the charge distribution of the $\mathrm{PAH}$ population under "cold neutral medium" conditions $\left(n_{\mathrm{H}} \approx 30 \mathrm{~cm}^{-3}, n_{e} \approx 0.03 \mathrm{~cm}^{-3}, T \approx 10^{2} \mathrm{~K}\right)$, Draine (2006) estimates that the timescale for a D atom to collide with a charged PAH is $\sim 2$ Myr. If no processes act to return $\mathrm{D}$ to the gas phase, $\mathrm{D}$ depletion by a factor of $e$ could therefore be achieved in only $2 \mathrm{Myr}$. In more diffuse regions, the timescale will be longer. For "warm neutral medium" conditions $\left(n_{\mathrm{H}} \approx 0.3 \mathrm{~cm}^{-3}, n_{e} \approx 0.03 \mathrm{~cm}^{-3}, T \approx 6000 \mathrm{~K}\right)$, a similar calculation gives a much longer D depletion time of $\sim 50 \mathrm{Myr}$. Therefore it appears that $\mathrm{D}$ depletion will be dominated by the cooler, denser regions where gas-grain collision rates are high. 
An important point is that most of the surface area available for deuterium depletion is provided by the population of free-flying PAHs, but this population is thought to contain only $C_{\mathrm{PAH}} / H_{\text {total }} \approx 60 \mathrm{ppm}$ (Li \& Draine 2001 ), or $\sim 1 / 3$ of the total aromatic material, with the remaining carbonaceous material in grains with radii $a \gtrsim 150 \AA$. How can the material in the larger grains become D-enriched? In denser regions, free-flying PAHs are expected to coagulate and become part of larger grains. The population of small PAHs is presumed to be replenished by grain fragmentation in grain-grain collisions. Thus there is steady interchange of material between PAHs and large grains. The timescale for a PAH to collide and coagulate with larger grains is $\sim 10 \mathrm{Myr}$ in the cold neutral medium, assuming that the larger grains have a total projected area per $\mathrm{H}$ nucleon of $10^{-21} \mathrm{~cm}^{2}$, and a characteristic velocity relative to the gas of $\sim 1 \mathrm{~km} / \mathrm{s}$, driven by MHD turbulence (Yan et al. 2004). In warm neutral medium conditions, this timescale becomes very long; once again, it appears that the exchange of matter between PAHs and larger grains is dominated by processes in the cold neutral medium. Therefore, the conditions are favorable for the time-dependent depletion of deuterium from the gas phase due to local events without changing $(\mathrm{D} / \mathrm{H})_{\text {total }}$ significantly by either astration or infall of less-astrated gas.

\section{TIME-DEPENDENT DEUTERIUM DEPLETION MODEL}

We now apply the deuterium depletion model to the three-regime interpretation of the $(\mathrm{D} / \mathrm{H})_{\text {gas }}$ data presented in Figure 1. The measurements of $(\mathrm{D} / \mathrm{H})_{\text {gas }}$ in the Local Bubble are for gas in warm clouds $(T \approx 7,000 \mathrm{~K}$ ) embedded in a hot gas bubble created by supernova events, which likely occured in the Lower Centaurus Crux subgroup of the Scorpio-Centaurus Association during the last 10 Myr (Maíz-Apellániz 2001). The Local Bubble was last reheated and presumably shocked by a supernova event 1-2 Myr ago (Lyu \& Bruhweiler 1996; Berghöfer \& Breitschwerdt 2002). If the Local Interstellar Cloud is representative (Redfield \& Linsky 2000), typical column densities for the warm clouds in the Local Bubble are $N(\mathrm{H} \mathrm{I}) \sim 10^{18} \mathrm{~cm}^{-2}$ (see Table 2 ). The origin of these clouds is uncertain. It is possible that they consist of material that has cooled after being heated by thermal conduction or turbulent mixing with hot gas. In this case some fraction of deuterium in grains might have been returned to the gas by thermal sputtering while the gas was sufficiently hot for this to be effective. Without knowledge of the thermal history of individual Local Bubble gas clouds, it is not possible to predict what values of $(\mathrm{D} / \mathrm{H})_{\text {gas }}$ are expected in this interpretation, but the observed value of $\sim 15 \mathrm{ppm}$ appears to be compatible with this picture. Alternatively, preexisting neutral clouds might have been shocked by low-velocity shocks driven into the clouds by a sudden increase in ambient pressure. Frisch et al. (1999) argue that the abundances of $\mathrm{Si}, \mathrm{Mg}$, and Fe in the Local Interstellar Cloud are consistent with grain processing through a 
shock with $\sim 100 \mathrm{~km} \mathrm{~s}^{-1}$. In this interpretation, grain-grain collisions would be expected to result in destruction of only a few percent of the refractory grain material like iron (Frisch \& Slavin 2003). Since the condensation temperature (Savage \& Sembach 1996) of Fe (1300 K) is much larger than for $\mathrm{C}(\sim 50 \mathrm{~K})$ to which the $\mathrm{D}$ is bound, we anticipate that grain-grain collisions, radiation, or other heating processes would strip D from grains before Fe. Since, as we will later argue, the total (gas and dust) value of $(\mathrm{D} / \mathrm{H})$ in the local Galactic disk is about $45 \%$ larger than $(\mathrm{D} / \mathrm{H})_{\text {LBgas }}$, we surmize that the shocks in the Local Bubble have only partially evaporated the deuterium-bearing grains that could have been formed in molecular clouds before the onset of star formation in the Association. As we shall see in $\S 6.1$, the modest depletion of deuterium in the Local Bubble corresponds to modest depletions of the refractory metals iron and silicon and, as described in $\S 2$, also oxygen.

Radio observations in the H I $21 \mathrm{~cm}$ line provide the best information on the properties and number density of individual diffuse H I clouds in the ISM. Typical diffuse H I clouds have column densities in the range $\log N(\mathrm{H} \mathrm{I})=18.8-19.8$ with a median column density $\log N($ H I $)=19.3$ (Lockman, Jahoda, \& McCammon 1986; Lockman 2002). Cox \& Reynolds (1987) cite a mean temperature for diffuse H I clouds of $~ 80 \mathrm{~K}$. They also say that, on average, the number of such clouds per $\mathrm{kpc}$ in the disk with column densities in excess of $N(\mathrm{H} \mathrm{I})$ is $5.7\left[N(\mathrm{H} \mathrm{I}) / 10^{20} \mathrm{~cm}^{-2}\right]^{-0.8}$. Using this formula, the number of clouds per kpc with $\log N(\mathrm{H} \mathrm{I})>19.3$ is about 20 , with a typical cloud separation of about $50 \mathrm{pc}$; however, the distribution should be very patchy.

Lines of sight extending beyond the Local Bubble should pass though either recently shocked gas, not recently shocked gas, or a mixture of such regions. According to our time-dependent depletion model, the highest measured values of $(\mathrm{D} / \mathrm{H})_{\text {gas }}$ should indicate recently shocked regions where $(\mathrm{D} / \mathrm{H})_{\text {gas }} \leq(\mathrm{D} / \mathrm{H})_{\text {total }}$, and the lines of sight with low values of $(\mathrm{D} / \mathrm{H})_{\text {gas }}$ should indicate regions that have not been shocked for a long time and thus have severe deuterium depletion. Lines of sight extending beyond the Local Bubble with the highest measured values of $(\mathrm{D} / \mathrm{H})_{\text {gas }-\mathrm{LB}}$, that is values above $21 \mathrm{ppm}$, include the lines of sight toward $\gamma^{2}$ Vel, Lan 23, WD 1034+001, Feige 110, and LSE 44.

Given the patchy distribution of diffuse H I clouds, lines of sight extending beyond the Local Bubble can include none, one, or many of these clouds. If there is, at most, one diffuse $\mathrm{H}$ I cloud in a line of sight, then $(\mathrm{D} / \mathrm{H})_{\text {gas }}$ should reflect the conditions of warm interstellar gas near the Sun that is partially ionized and likely has not had sufficient time to deplete an appreciable fraction of deuterium onto dust after the last shock. However, when a line of sight passes through several or many diffuse H I clouds, the composite value of $(\mathrm{D} / \mathrm{H})_{\text {gas }}$ should be dominated by the abundances in these clouds where gas-phase deuterium can be highly depleted onto the cold grains even when the gas is not cold. Thus lines of sight in the 
intermediate regime $(\log N(\mathrm{H} \mathrm{I})=19.2-20.7)$ could contain little or no unshocked $\mathrm{H}$ I gas or be dominated by such material, leading to a wide range of $(\mathrm{D} / \mathrm{H})_{\text {gas }}$ values.

HST and Copernicus spectra of $\gamma^{2}$ Vel and diffuse $\mathrm{H} \alpha$ emission near the star show highly ionized gas (including O VI) along its line of sight from which Fitzpatrick \& Spitzer (1994) and Reynoso \& Dubner (1997) infer an H II region of path length 100-150 pc produced by a wind-blown superbubble and photoionizing radiation from $\gamma^{2}$ Vel and other massive stars. These conditions of strong ultraviolet radiation and recent supernova-produced shocks could remove deuterium from the mantles of dust grains, leading to the observed high value of $(\mathrm{D} / \mathrm{H})_{\text {gas }}$. The ionization along the other lines of sight with high $(\mathrm{D} / \mathrm{H})_{\text {gas }}$ is not yet characterized.

For lines of sight with larger column densities $(\log N(\mathrm{H} \mathrm{I})>20.7)$, we expect that most of the column density will be contributed by cool $\mathrm{H}$ I gas, and the overall $(\mathrm{D} / \mathrm{H})_{\text {gas }}$ value should be close to the low $(\mathrm{D} / \mathrm{H})_{\text {gas }}$ values prevalent in the cool H I phase. Measurements of $(\mathrm{D} / \mathrm{H})_{\text {gas }}$ along as many long lines of sight as possible with FUSE and other instruments are needed to test this hypothesis. For a discussion of possible line saturation effects see $\S$ 6.3.2.

\section{TESTING THE DEUTERIUM DEPLETION MODEL}

We now pose two tests to determine whether or not deuterium depletion is a valid model for explaining the wide range of $(\mathrm{D} / \mathrm{H})_{\text {gas }}$ values for lines of sight extending beyond the Local Bubble. While neither test is conclusive, the statistical trends identified by these tests taken together make for a strong case. One problem in this analysis is that the lines of sight to the very bright stars analyzed with data from Copernicus have larger uncertainties in $(\mathrm{D} / \mathrm{H})_{\text {gas }}$ than the lines of sight to a different group of stars studied using IMAPS or FUSE spectra. IMAPS reobserved the Copernicus stars $\gamma^{2}$ Vel, $\zeta$ Pup, and $\delta$ Ori, but FUSE was unable to reobserve any of the Copernicus stars to obtain more accurate $\mathrm{D} / \mathrm{H}$ measurements. A rough estimate of the improvement in the measurements obtained with IMAPS and FUSE comes from a comparison of the $(\mathrm{D} / \mathrm{H})_{\text {gas }}$ values for $\gamma^{2}$ Vel: the Copernicus result (York \& Rogerson 1976) was $17.8_{-6.6}^{+10.4} \mathrm{ppm}$, whereas the IMAPS result (Sonneborn et al. 2000), which we use in our analysis, was $21.8 \pm 2.1 \mathrm{ppm}$. Although the two results are consistent within the Copernicus error bars, the IMAPS value is $2.1 \sigma$ (IMAPS error bars) above the value obtained from Copernicus spectra. Vidal-Madjar et al. (1977) used Copernicus data to infer two velocity components in the line of sight to $\zeta$ Pup: in component $1(\mathrm{D} / \mathrm{H})_{\text {gas }}=22 \mathrm{ppm}$ with no error estimate, and in component $2(\mathrm{D} / \mathrm{H})_{\text {gas }}=23_{-3}^{+7} \mathrm{ppm}$. The high spectral resolution and S/N of the IMAPS data allowed Sonneborn et al. (2000) to resolve or nearly resolve the complex velocity structure for this line of sight and to infer $(\mathrm{D} / \mathrm{H})_{\mathrm{gas}}=14.0 \pm 2.3 \mathrm{ppm}$, which 
is well below the Copernicus result. On the other hand, for the $\delta$ Ori line of sight there is good agreement between the Copernicus result of $7.5 \pm 2.3 \mathrm{ppm}$ (Laurent, Vidal-Madjar, \& York 1979) and the IMAPS result of $7.4_{-0.9}^{+1.2} \mathrm{ppm}$ (Jenkins et al. 1999). These comparisons suggest that we should be careful when including Copernicus data together with the more accurate recent data. In particular, the Copernicus data for the lines of sight to $\alpha$ Vir and $\mu \mathrm{Col}$ have uncertainties in $(\mathrm{D} / \mathrm{H})_{\text {gas }}$ that are near $100 \%$. We have chosen to include all of the Copernicus data, which will clutter the subsequent figures somewhat, but in computing correlations we weight each of the data points by the inverse of the $1 \sigma$ range in $(D / H)_{\text {gas. }}$ In this way, the recent data with small measurement uncertainties will dominate the correlation fits.

In Figures 3-8 we compare $(\mathrm{D} / \mathrm{H})_{\text {gas }}$ vs. other observables along the same lines of sight. Except for Figure 5, we do not use $(\mathrm{D} / \mathrm{H})_{\text {gas-LB }}$ because we have no reliable way of removing the Local Bubble foreground from the other quantity that $(\mathrm{D} / \mathrm{H})_{\text {gas }}$ is being compared with for most lines of sight. For all of the correlation tests, we compute weighted least-squares linear fits, $\log [N(\mathrm{D} \mathrm{I}) / N(\mathrm{H} \mathrm{I})]=\mathrm{a}+\mathrm{bD}(\mathrm{Fe})$ or $\mathrm{a}+\mathrm{bD}(\mathrm{Si})$, to the observed data points by allowing all of the data quantities, $N(\mathrm{H} \mathrm{I}), N(\mathrm{D} \mathrm{I})$, and either $N(\mathrm{Fe}$ II) or $N(\mathrm{Si}$ II), to vary randomly within their $1 \sigma$ error range. We compute a and b parameters for 1000 such realizations and then fit Gaussians to the distributions of the a and b parameters. Table 5 lists the mean values of the a and b parameters and their $1 \sigma$ uncertainties, which are the half-widths of the corresponding Gaussian distributions. The relatively small $1 \sigma$ uncertainties in the $\mathrm{b}$ slope parameters for these fits demonstrates that correlations are real despite the correlation of the errors in the $\mathrm{x}$-axis and $\mathrm{y}$-axis quantities (see $\$ 6.3 .6$ for a different approach). For each plot we also apply the Spearman rank correlation test ${ }^{4}$, which provides an unbiased means for determining whether the null hypothesis of no correlation can be rejected for the unweighted data points. Column 9 in Table 5 gives the Spearman rank correlation coefficient, and column 10 gives the two-sided significance of its deviation from zero. Small values of this significance parameter indicate significant correlation. The quantity in parenthesis is the number of standard deviations by which the significance parameter deviates from its null-hypothesis value. These quantities are listed in Table 5 for the various tests described below.

\footnotetext{
${ }^{4}$ We use the IDL library routine R_CORRELATE to compute the Spearman $(\rho)$ rank correlation test. The description of this test is from the IDL Reference Guide.
} 


\subsection{First Test: Correlation with Refractory Metal Depletions}

Draine $(2004,2006)$ proposed that a good test of whether deuterium depletion onto dust is responsible for the observed variations in $(\mathrm{D} / \mathrm{H})_{\text {gas }}$ is to see whether there is a positive correlation of depletion of metals like Fe, $\mathrm{Si}$, and $\mathrm{Ti}$ with $(\mathrm{D} / \mathrm{H})_{\text {gas }}$. Therefore, our first test of the validity of the deuterium depletion model is to determine whether for the same lines of sight, the observed $(\mathrm{D} / \mathrm{H})_{\text {gas }}$ ratios correlate with the depletion of refractory metals, that is, elements that easily condense onto interstellar grains because of their high chemical bonding energies. In the low density ISM, the primary source of grain erosion is due to highenergy-sputtering processes that occur in supernova-generated shock waves (Jones 2000). Since iron in grains is very resistant to sputtering, small values of the iron depletion would indicate significant processing of the grains that, according to the model, would predict that most or all of the deuterium has been removed from the grains. Gas-phase iron abundances can be measured accurately in the ISM, as STIS and FUSE spectra both provide accurate measurements of the dominant ionization stage, $\mathrm{Fe}^{+}$, for both cold and warm gas. The depletion of iron, $\mathrm{D}(\mathrm{Fe})=\log _{10}\left[(\mathrm{Fe} / \mathrm{H})_{\text {gas }} /(\mathrm{Fe} / \mathrm{H})_{\odot}\right]$, varies from typically -1.2 in warm diffuse clouds to typically -2.2 in cool diffuse clouds (Savage \& Sembach 1996; Jenkins 2004). Very small iron depletions would indicate that the dust grains have been vaporized, depositing iron and deuterium from the dust back into the gas phase. Thus, very large negative values of $\mathrm{D}(\mathrm{Fe})$ should correlate with low $(\mathrm{D} / \mathrm{H})_{\text {gas }}$, if the deuterium depletion model is valid. Conversely, small negative values of $\mathrm{D}(\mathrm{Fe})$ should correlate with high $(\mathrm{D} / \mathrm{H})_{\text {gas }}$ measurements. Column (8) in Table 3 lists the values of $\mathrm{D}(\mathrm{Fe})$ obtained using $N(\mathrm{Fe}$ II) either from the reference given in column (11) of Table 2 or from the data compilations of Jenkins, Savage, \& Spitzer (1986) and Redfield \& Linsky (2002). In a few cases, we have revised slightly the values of $N(\mathrm{Fe}$ II) as a result of more accurately determined f-values (Howk et al. 2000) for the optically thin lines that most strongly constrain the Fe II column density. These revisions range from +0.04 dex for $\mathrm{BD}+39^{\circ} 3226$ to -0.04 for $\theta$ Car. We then compute $\mathrm{D}(\mathrm{Fe})$ using our tabulated value of $N(\mathrm{H} \mathrm{I})$. The undepleted value for iron is assumed to be the solar abundance ratio, $\log (\mathrm{Fe} / \mathrm{H})_{\odot}=-4.55 \pm 0.05$ (Asplund et al. 2005), determined using a 3D hydrodynamical model of the solar atmosphere.

Most lines of sight inside the Local Bubble show iron depletions in the range of $-0.7>$ $\mathrm{D}(\mathrm{Fe})>-1.3$, which are typical for warm diffuse clouds. Two lines of sight (36 Oph and G191-B2B) show smaller iron depletions and have values of $(\mathrm{D} / \mathrm{H})_{\text {gas }}$ that are typical for the Local Bubble (14.1 $\pm 5.8 \mathrm{ppm}$ and $16.6 \pm 4.1 \mathrm{ppm}$, respectively). There is no measurement of $\mathrm{D}(\mathrm{Fe})$ for the line of sight to $\beta$ Cet, but magnesium is undepleted for this line of sight (Piskunov et al. 1997). Also, silicon, another refractory metal, is not depleted on this line of sight. From Jenkins (2004), one sees that $\mathrm{D}(\mathrm{Fe}) \approx \mathrm{D}(\mathrm{Si})-0.8$, so we might estimate $\mathrm{D}(\mathrm{Fe}) \approx-0.8$ for the line of sight to $\beta$ Cet, which also has the highest value of $(\mathrm{D} / \mathrm{H})_{\mathrm{gas}}=$ 
$21.9_{-6.8}^{+5.2} \mathrm{ppm}$ in the Local Bubble. This connection of high $(\mathrm{D} / \mathrm{H})_{\text {gas }}$ with minimal metal depletion is as predicted by the deuterium-depletion model.

In Figure 3, we plot $(\mathrm{D} / \mathrm{H})_{\text {gas }}$ vs. $\mathrm{D}(\mathrm{Fe})$ for all 38 lines of sight in Table 3 with measured $\mathrm{D}(\mathrm{Fe})$ values (excluding the upper and lower limits). Figure 3 shows a clear trend of decreasing $(\mathrm{D} / \mathrm{H})_{\text {gas }}$ with increasing iron depletion, especially for very large Fe depletions, $\mathrm{D}(\mathrm{Fe})<-1.5$. The solid line in the plot is the least-squares-weighted linear fit to the data as described above. We fit the data with an equation of the form $(\mathrm{D} / \mathrm{H})_{\text {gas }}=(\mathrm{a} \pm 1 \sigma)+$ $(\mathrm{b} \pm 1 \sigma) \mathrm{D}(\mathrm{Fe})$. For this fit, the parameters are $\mathrm{a}=30.00 \pm 1.60$ and $\mathrm{b}=13.04 \pm 1.15$. The Spearman rank correlation test ${ }^{5}$ of the unweighted data points shows a highly significant correlation parameter and rejects the null hypothesis with $2.9 \sigma$ significance. These parameters are listed in Table 5. While there are five data points that lie above the linear fit, the statistical correlation of large Fe depletions with small values of $(\mathrm{D} / \mathrm{H})_{\text {gas }}$ supports the deuterium-depletion hypothesis ${ }^{6}$. In $\S 6.3$ we discuss possible causes of scatter about the trend line, which in particular make $\gamma^{2}$ Vel no longer appear anomalous.

Figure 4 is similar to Figure 3 but with the depletion of silicon $\mathrm{D}(\mathrm{Si})$ for the $\mathrm{x}$ axis. The values of $\mathrm{D}(\mathrm{Si})$, listed in column (9) of Table 3 , were obtained using $N(\mathrm{Si}$ II) from the references and the tabulated values of $N(\mathrm{H} \mathrm{I})$. More significant revisions in $N(\mathrm{Si}$ II) are needed than for Fe II as the f-values used for the weak Si II 1020.7 and $1808.0 \AA$ lines have changed greatly since the Copernicus and IUE eras. We use the Morton (2003) f-values for these lines, which are based on laboratory measurements. The changes in $N(\mathrm{Si}$ II) range from +0.43 dex for $\mathrm{BD}+39^{\circ} 3226$ to -0.02 dex for $\beta$ CMa with most changes significantly positive. We compute the silicon depletion assuming the solar abundance of silicon as $\log$ $(\mathrm{Si} / \mathrm{H})_{\odot}=-4.49 \pm 0.04$ (Asplund et al. 2005). We find that $\mathrm{D}(\mathrm{Si})$, like $\mathrm{D}(\mathrm{Fe})$, shows a clear trend of increasing depletion with lower deuterium abundance. Our weighted least-squares fit to the 20 data points (excluding upper limits) is of the form $(\mathrm{D} / \mathrm{H})_{\text {gas }}=(\mathrm{a} \pm 1 \sigma)+(\mathrm{b} \pm 1 \sigma)$ $\mathrm{D}(\mathrm{Si})$. The Spearman rank correlation test of the unweighted data points rejects the null hypothesis with $1.4 \sigma$ significance. The parameters for this fit are given in Table 5.

In their recent paper, Prochaska, Tripp, \& Howk (2005) find a similar correlation of $(\mathrm{D} / \mathrm{H})_{\text {gas }}$ with the depletion of titanium, which also has a high condensation temperature

\footnotetext{
${ }^{5}$ In $\S 6.3 .5$ we redo this analysis using only the data obtained with the STIS, GHRS, IMAPS, and FUSE instruments. The effect is to remove the data points with the largest errors, leaving data points that mostly have similar errors and thus similar weights. The result for 26 data points is a similar linear fit that rejects the null hypothesis of no correlation with $2.3 \sigma$ significance.

${ }^{6}$ There is no theoretical reason for assuming that the best-fit relation should be linear, or that the D depletion and Fe depletion should be tightly correlated with one another.
} 
for the lines of sight to seven of the stars listed in Table 2 located beyond the Local Bubble. Although Ti II has a similar ionization energy to H I and D I, the velocity profiles of the Ti II lines do not track very well the profiles of O I $1335 \AA$ for the line of sight to HD 195965, perhaps due to different depletion levels as suggested by Prochaska, Tripp, \& Howk (2005). Although the correlations of $(\mathrm{D} / \mathrm{H})_{\text {gas }}$ with $\mathrm{D}(\mathrm{Fe})$ and $\mathrm{D}(\mathrm{Si})$ are significant, the scatter that we have seen in Figure 3 and Figure 4 suggests that different physical processes are responsible for the depletions of $\mathrm{D}$ compared to the metals and most likely among the different metals. This could be the cause of the differences between the O I and Ti II line profiles for HD 195965 and would suggest significant scatter in plots of $(\mathrm{D} / \mathrm{H})_{\text {gas }}$ vs $\mathrm{D}(\mathrm{Ti})$.

Wakker \& Mathis (2000), Jenkins et al. (1986), and others have demonstrated that the depletion of iron and other refractory elements becomes larger with increasing $N(\mathrm{H} \mathrm{I})$, or increasing mean hydrogen number density, $\left\langle n_{\mathrm{H}}\right\rangle$, along the line of sight. This relation is generally explained in terms of the increasing contribution of quiescent cold clouds, with their large values of $N(\mathrm{H} \mathrm{I})$ and greater metal depletions, to the total $N(\mathrm{H} \mathrm{I})$ when a line of sight passes through one or more such cold clouds. See also Oliveira et al. (2006) for a study of the correlation of $(\mathrm{D} / \mathrm{H})_{\text {gas }}$ with $\left\langle n_{\mathrm{H}}\right\rangle$ along the line of sight. The correlations of $(\mathrm{D} / \mathrm{H})_{\text {gas }}$ with $\mathrm{D}(\mathrm{Fe})$ shown in Figure 3 and with $\mathrm{D}(\mathrm{Si})$ shown in Figure 4 are consistent with $\mathrm{D}, \mathrm{Fe}$, and $\mathrm{Si}$ being depleted onto grains in cold clouds.

\subsection{Second Test: $H_{2}$ Rotational Excitation}

The second empirical test of the deuterium depletion hypothesis is whether or not there is a correlation of $(\mathrm{D} / \mathrm{H})_{\text {gas }}$ with $\mathrm{H}_{2}$ rotational temperature. Table 6 summarizes the molecular hydrogen data for the fourteen lines of sight with $(\mathrm{D} / \mathrm{H})_{\text {gas }}$ measurements. When both $N\left(\mathrm{H}_{2}\right)$ and $N(\mathrm{HD})$ are measured for a given line of sight, we include these molecules in the value of $(\mathrm{D} / \mathrm{H})_{\text {gas }}$. With some exceptions, for column densities exceeding $\log N(\mathrm{H}) \approx 20.7$ (in our Galaxy), many $\mathrm{H}_{2}$ spectral lines become optically thick, and the resulting shielding of the FUV radiation field decreases the photodissociation rate leading to a high molecular hydrogen fraction, $f\left(\mathrm{H}_{2}\right)$ (Spitzer \& Jenkins 1975; Savage et al. 1977). Under these conditions cloud densities can be sufficiently high that the $\mathrm{H}_{2}$ rotational excitation temperature $T_{01}$, derived from the $\mathrm{J}=1$ and $\mathrm{J}=0$ levels of $\mathrm{H}_{2}$, measures the gas kinetic temperature. In their analysis of FUSE spectra of 129 sightlines to O and B stars in the Galactic plane, Shull et al. (2005) find that $T_{01}=86 \pm 20 \mathrm{~K}$. Gillmon et al. (2006) find that $T_{01}=124 \pm 8 \mathrm{~K}$ characterizes lines of sight through the halo. For the Galactic plane data set, $\log \left[N(\mathrm{H} \mathrm{I})+2 N\left(\mathrm{H}_{2}\right)\right] \geq 20.9$, while our data set is mostly for smaller column densities. The three lines of sight in Table 6 with the highest column densities overlap the Shull et al. (2005) data set, and their values 
of $T_{01}$ are consistent with the Shull et al. (2005) range as expected.

Eleven of the fourteen lines of sight listed in Table 6 have column densities $\log [N(\mathrm{H} \mathrm{I})+$ $2 N\left(\mathrm{H}_{2}\right)$ ] much smaller than 20.9 and, for the most part, values of $T_{01}$ much larger than $86 \mathrm{~K}$. Since this is a new region of parameter space to explore, we decided to test for a correlation between $T_{01}$ and $(\mathrm{D} / \mathrm{H})_{\text {gas }}$. Given that there is essentially no $\mathrm{H}_{2}$ located inside the Local Bubble, we plot $(\mathrm{D} / \mathrm{H})_{\text {gas }-\mathrm{LB}}$ vs. $\mathrm{T}_{01}$. The data shown in Figure 5 do indeed show a correlation with one discrepant point, $\delta$ Ori, which is located behind a cloud that has been excited by stellar winds or explosive events (Jenkins et al. 2000b). Our weighted leastsquares fit to the 16 data points is of the form $(\mathrm{D} / \mathrm{H})_{\text {gas }-\mathrm{LB}}=(\mathrm{a} \pm 1 \sigma)+(\mathrm{b} \pm 1 \sigma) \log \left(\mathrm{T}_{01}\right)$. The Spearman rank correlation test of the unweighted data rejects the null hypothesis with $2.2 \sigma$ significance, indicating a credible correlation. The fit parameters are listed in Table 5. Although the correlation is only at a $2.2 \sigma$ confidence level, we should ask why there should be any correlation at all. Since the lines of sight are generally inhomogeneous, most of the molecular hydrogen along a given line of sight should be located in cool or cold clouds and thus not be cospatial with most of the atomic hydrogen and deuterium. Thus the properties of $\mathrm{H}_{2}$, such as $T_{01}$, could be independent of the depletions of D, Fe, and Si averaged over the line of sight. Nevertheless, we have found a correlation of $T_{01}$ with $(\mathrm{D} / \mathrm{H})_{\text {gas }}$ and therefore indirectly with the depletions of $\mathrm{Fe}$ and $\mathrm{Si}$. Furthermore, for $N(\mathrm{H})<20.7, T_{01}$ should not be a good measure of the gas temperature but is rather determined by the balance of excitations and de-excitations of the lower levels of $\mathrm{H}_{2}$ with collisions likely unimportant. We note that in Table 6 all of the lines of sight with high values of $T_{01}$ have very small fractional abundances of $\mathrm{H}_{2}, f\left(\mathrm{H}_{2}\right)$. For these lines of sight there is likely very little cold gas and thus less depletion of deuterium and metals. We do not know whether this is a good explanation for the correlation of $(\mathrm{D} / \mathrm{H})_{\text {gas }}$ with $T_{01}$ shown in Figure 5 . Observations of more lines of sight with low values of $N(\mathrm{H})$ but containing $\mathrm{H}_{2}$ and further theoretical work are needed to verify the correlation and to explore other possible explanations.

\subsection{Possible Causes for Scatter and Discrepant Lines of Sight}

Figure 3 shows that five lines of sight $\left(\gamma^{2}\right.$ Vel, Lan 23, WD 1034+001, PG 0038+199, and TD1 32709) have high values of $(\mathrm{D} / \mathrm{H})_{\text {gas }}$ as compared to the general trend of the other lines of sight. In Figure 5, the line of sight to $\delta$ Ori has a low value of $(\mathrm{D} / \mathrm{H})_{\text {gas-LB }}$ compared to the trend line. What could explain these discrepant data points and, for that matter, the scatter of data about the least-squares trend lines? We list here several considerations that may play important roles in explaining the scatter, but this list may not be complete: 


\subsubsection{Inhomogeneous Lines of Sight}

Until now, we have assumed that the properties of interstellar gas along a given line of sight have constant values inside the Local Bubble and then a different set of constant values beyond the Local Bubble. This very simple approximation is invalid for most lines of sight that have been studied with sufficient spectral resolution to identify the different velocity components and their individual properties. If the properties of interstellar gas in the different components are significantly different, for example in temperature, ionization (H II vs. H I regions), and density, then different observables may be formed preferentially in different locations and thus not correlate well. One example is the correlation of $(\mathrm{D} / \mathrm{H})_{\text {gas }-\mathrm{LB}}$ with the temperature of $\mathrm{H}_{2}$, as $\mathrm{H}_{2}$ could be present in only one of the components along the lines of sight. For lines of sight with low $\mathrm{H}_{2}$ column densities, $\mathrm{H}_{2}$ could be found in relatively warm gas associated with $\mathrm{H}$ I and neutral metals. For example, high-resolution spectra of $\delta$ Ori obtained with the IMAPS instrument show $\mathrm{H}_{2}$ line profiles with similar velocity structure as N I, O I, and D I (Jenkins et al. 1999, 2000b). This could explain why $T_{01}$ is large for the the $\delta$ Ori line of sight.

One way of estimating the depletion of an element in a particular velocity component without information on $N(\mathrm{H} \mathrm{I})$ for that component is to reference the gas-phase column density of the element to another element like sulphur, which is not significantly depleted in $\mathrm{H}$ I regions. Howk et al. (1999) have used this approach to derive $\mathrm{D}(\mathrm{Fe})$ and $\mathrm{D}(\mathrm{Si})$ for Component 1 in the line of sight to $\mu \mathrm{Col}$ using GHRS echelle spectra. This low-velocity component, also called Component A by Shull \& York (1977), contains most of the neutral gas and $\mathrm{H}_{2}$ along the line of sight. Tables 2, 3, and 6 include the (Howk et al. 1999) data for Component 1 .

\subsubsection{Line Saturation}

For complex lines of sight with large $N(\mathrm{H} \mathrm{I})$, it is important to measure $N(\mathrm{D}$ I) from the higher lines in the Lyman series to avoid line saturation that could lead to underestimates in $N\left(\mathrm{D}\right.$ I) and therefore in $(\mathrm{D} / \mathrm{H})_{\text {gas }}$. The $20 \mathrm{~km} \mathrm{~s}^{-1}$ spectral resolution of FUSE is inadequate to resolve the $\mathrm{D}$ lines or to separate velocity components in the line. In particular, narrow components from cold gas could be saturated but not recognizable at the resolution of FUSE. We have argued in $\S 5$ that the low values of $(\mathrm{D} / \mathrm{H})_{\text {gas }}$ for lines of sight beyond the Local Bubble could be due to the dominant role of cool diffuse clouds in which deuterium is largely depleted. Such clouds would have narrow absorption features observable in metal lines. Indeed, Redfield \& Linsky (2002) list a number of lines of sight with Ca II absorption

features with $b<1.5 \mathrm{~km} \mathrm{~s}^{-1}$. The chances of this occuring grow with increasing $N(\mathrm{H} \mathrm{I})$, 
but measurements of $N(\mathrm{D}$ I) in high members of the Lyman series, which require high $\mathrm{S} / \mathrm{N}$ data, decrease the probability that line saturation will occur. One can test for D line saturation by looking for an increase in $N(\mathrm{D}$ I) toward higher lines in the Lyman series or from a curve of growth analysis (e.g., Friedman et al. 2002). Also, high resolution spectra of interstellar metal lines can identify velocity features that could have saturated D absorption. The blending of adjacent Lyman lines with each other and with the many $\mathrm{H}_{2}$ lines will eventually set a limit to the largest value of $N(\mathrm{H}$ I) for which one can measure unsaturated D Lyman lines, but this should not occur until $\log N(\mathrm{H} \mathrm{I})$ is considerably larger than 21.0 (Jenkins 1996).

\subsubsection{Ionization Corrections}

Jenkins et al. (1986) and Jenkins (2004) have argued that anomalously small metal depletions compared to typical lines of sight with the same $N(\mathrm{H} \mathrm{I})$ could be due to H II regions where hydrogen is partially ionized but metals with second ionization potentials greater than $13.58 \mathrm{eV}$, such as iron, silicon, and phosphorus (Lehner et al. 2004), remain mostly singly ionized. Positive values of $\mathrm{D}(\mathrm{Si})$ are consistent with $\mathrm{Si}^{+}$being the dominant ionization state and $\mathrm{H}$ being partially ionized. A good example of this is the line of sight to $\beta$ CMa. If we assume that essentially all of the hydrogen is $\mathrm{H} \mathrm{I}$ and all of the silicon and iron are singly ionized for this line of sight, then $\mathrm{D}(\mathrm{Si})=+0.41 \pm 0.16$, and $\mathrm{D}(\mathrm{Fe})=-0.30 \pm 0.16$ (Gry, York, \& Vidal-Madjar 1985; Dupin \& Gry 1998). However, along this line of sight hydrogen is mostly ionized (Gry, York, \& Vidal-Madjar 1985). In their analysis of this line of sight, Dupin \& Gry (1998) identify four velocity components with most of the H I column density in Component C. We therefore list in Table 3 the depletions for Component $\mathrm{C}$ that include all ionization stages for hydrogen, silicon, and iron, $\mathrm{D}(\mathrm{Si})=+0.04_{-0.14}^{+0.20}$ and $\mathrm{D}(\mathrm{Fe})$ $=-0.61_{-0.14}^{+0.20}$ (Dupin \& Gry 1998). The line of sight to $\beta$ Cet also has a positive value for the silicon depletion, $\mathrm{D}(\mathrm{Si})=+0.30 \pm 0.41$. Redfield \& Linsky (2004b) showed that one of the two velocity components toward $\beta$ Cet has a very high temperature, $T=12,400 \pm 2,800 \mathrm{~K}$, likely indicating partial ionization of hydrogen. Thus ionization corrections of presently unknown size should be applied to $\mathrm{D}(\mathrm{Si})$ for this line of sight, which would move its data point to the right in Figure 4. The high temperature of the gas toward $\beta$ Cet is likely due to a recent shock, which is consistent with the high value of $(\mathrm{D} / \mathrm{H})_{\text {gas }}=21.9_{-6.8}^{+5.2} \mathrm{ppm}$ for this line of sight.

The $\gamma^{2}$ Vel line of sight has seven velocity components - three are H II regions, likely produced by ionization from the Wolf-Rayet star in the binary system, and four are H I regions (Fitzpatrick \& Spitzer 1994). The H I regions contribute $89 \%$ of $N(\mathrm{Fe}$ II), but the 
fractional ionization of hydrogen and $N(\mathrm{H} \mathrm{I})$ in each component is not known. The iron depletion listed in Table 3 was therefore computed from the line-of-sight integrated values of $N(\mathrm{H} \mathrm{I})$ and $N(\mathrm{Fe} \mathrm{II})$, rather than from the column densities in the $\mathrm{H}$ I components. Inclusion of the unknown $N(\mathrm{H} \mathrm{II})$ would shift $\mathrm{D}(\mathrm{Fe})$ to the right in Figure 3. Since sulfur is not usually depleted, we estimate that $72 \%$ of the total hydrogen column density is located in the H II regions by summing the column densities of S I, S II, and S III in each velocity component. Although the inclusion of this amount of ionized hydrogen would shift $\mathrm{D}(\mathrm{Fe})$ for $\gamma^{2}$ Vel by 0.55 dex to the right in Figure 3, an unknown but considerable amount of Fe could be doubly ionized, shifting $\mathrm{D}(\mathrm{Fe})$ by an unknown amount to the left. Realistic calculations of the ionization of $\mathrm{H}$ and $\mathrm{Fe}$ are needed to address this problem.

Ionization corrections can be important for lines of sight with small values of $N(\mathrm{H} \mathrm{I})$ because ionizing radiation can then penetrate the gas and preferentially ionize $\mathrm{H}$ compared to $\mathrm{Fe}^{+}$and $\mathrm{Si}^{+}$. For example, Frisch \& Slavin (2003) find in their ionization equilibrium models for the nearby ISM that hydrogen is about $30 \%$ ionized while $96.5 \%$ of Fe is Fe II and $99.6 \%$ of $\mathrm{Si}$ is Si II. To avoid lines of sight where such ionization corrections are likely important, we reconsider the correlation of $(\mathrm{D} / \mathrm{H})_{\text {gas }}$ with $\mathrm{D}(\mathrm{Fe})$ and $\mathrm{D}(\mathrm{Si})$ but now remove the data for lines of sight with $\log N(\mathrm{H} \mathrm{I})<19.0$. This removes all but two of the Local Bubble lines of sight. In Figure 6, we plot $(\mathrm{D} / \mathrm{H})_{\text {gas }}$ vs. $\mathrm{D}(\mathrm{Fe})$ for the 24 data points that meet this criterion. The slope is now steeper than for the full data set (see Table 5). The Spearman rank correlation test rejects the null hypothesis of no correlation with $2.6 \sigma$ significance.

Figure 7 is similar to Figure 4, except that we remove the data points with $\log N(\mathrm{H} \mathrm{I})<$ 19.0 and plot $(\mathrm{D} / \mathrm{H})_{\text {gas }}$ vs. $\mathrm{D}(\mathrm{Si})$ for the 11 data points that meet this criterion. The slope is nearly the same as for the full data set (see Table 5). The Spearman rank correlation test rejects the null hypothesis of no correlation with $2.1 \sigma$ significance. The data are in excellent agreement with the linear fit, except for the one uncertain data point, $\mu$ Col.

The removal of lines of sight that are most likely affected by ionization corrections leads to steeper correlations of $(\mathrm{D} / \mathrm{H})_{\text {gas }}$ with $\mathrm{D}(\mathrm{Fe})$ and $\mathrm{D}(\mathrm{Si})$, fewer discrepant points, and tighter correlations. The remaining scatter could be due to measurement errors (especially for some of the Copernicus data), inhomogeneous lines of sight, and different grain compositions. The ionization of $\mathrm{H}$ and $\mathrm{D}$ should be the same as they share the same bound-free continuum.

\subsubsection{Different Grain Compositions}

One should not expect a simple one-to-one relation between $(\mathrm{D} / \mathrm{H})_{\text {gas }}$ and $\mathrm{D}(\mathrm{Fe})$ or $\mathrm{D}(\mathrm{Si})$ along a given line of sight, because deuterium and different metals may be depleted 
onto different types of grains or be located preferentially in different layers of the same grain. Many authors (e.g., Spitzer \& Fitzpatrick 1993) have argued that grains typically have hardy cores but more easily destroyed mantles. For example, iron may be concentrated in the grain cores and some deuterium concentrated in PADs either free-flying or incorporated into larger grains. Jones et al. (1994) estimated the destruction timescale for grains by supernova-driven shocks to be $2-3 \times 10^{8}$ yr but the timescale for the erosion of grain mantles by weaker shocks is much shorter. We note that the five lines of sight that lie above the trend line in Figure 3 $\left(\gamma^{2}\right.$ Vel, Lan 23, WD 1034+001, PG 0038+199, T1 32709) also have the highest values of $(\mathrm{D} / \mathrm{H})_{\text {gas }}$ (see Figure 1). (Feige 110, the fourth line of sight with a high value of $(\mathrm{D} / \mathrm{H})_{\text {gas }}$, does not have $\mathrm{D}(\mathrm{Fe})$ or $\mathrm{D}(\mathrm{Si})$ measurements.) Clearly, there is no simple one-to-one relationship between $(\mathrm{D} / \mathrm{H})_{\text {gas }}$ and metal depletion, although there are the general trends indicated by the least-squares trend lines. In Figure 6, where we have considered only lines of sight with $\log N(\mathrm{H} \mathrm{I})>19.0$ to minimize ionization corrections, all of the five high lines of sight are still present.

In Figure 3 the dashed line is drawn parallel to the least-squares fit to all of the lines of sight (solid line) but arbitrarily displaced upwards by $8.5 \mathrm{ppm}$. The dashed line is a good fit to the five high data points. The high values of $(\mathrm{D} / \mathrm{H})_{\text {gas }}$ for these five lines of sight could be explained by either a smaller than usual percentage of the grains being composed of carbon and thus few sites available for deuterium to deplete on or the evaporation of deuterium that was in grain mantles by weak shocks. If the slope of the dashed line is real and not an artifact of the few data points, then there is support for a smaller than usual percentage of carbon grains in these lines of sight. Since the condensation temperature for carbon is much smaller than that for iron (typically in the form of oxides) (Sofia, Cardelli, \& Savage 1994; Savage \& Sembach 1996), a low percentage of carbon grains compared to iron grains is likely for some lines of sight.

\subsubsection{Difficulty of Measuring N(H I)}

The most difficult column density to measure accurately is usually hydrogen because the Lyman lines are very optically thick, they must be measured against an uncertain stellar background (Lyman absorption or emission lines), and they are very broad, which requires interpolation over a large wavelength range. The hydrogen column densities cited in Table 2 are based on careful analyses that consider these and other effects. The most accurate values of $N(\mathrm{H} \mathrm{I})$ are generally obtained from analyses of STIS, GHRS, and FUSE spectra. We note that $N(\mathrm{H} \mathrm{I})$ for the Feige 110 line of sight was obtained from the analysis of one $I U E$ spectrum. Since the Lyman- $\alpha$ line lies near the end of the $I U E$ spectrum where the 
sensitivity is low and the echelle spectral orders are close together, it is difficult measure the background and continuum. While there is no evidence that the value of $N(\mathrm{H} \mathrm{I})$ listed in Table 2 is more uncertain than the cited error bars, the Feige 110 line of sight should be reobserved with a better spectrograph when feasible. The Lan 23 line of sight should also be reobserved as $N(\mathrm{H} \mathrm{I})$ was obtained from a noisy low dispersion $E U V E$ spectrum rather than from Lyman line spectra. To test whether the less reliable values of $N(\mathrm{H} \mathrm{I})$ are biasing our conclusions, we plot in Figure 8 only the data points for which $N(\mathrm{H} \mathrm{I})$ was obtained from STIS, GHRS, or FUSE. The fit to the data and the parameters characterizing the fit listed in Table 5 are similar to the results obtained when we included all of the data in Figure 3.

\subsubsection{Removing a Possible Correlation from the Plots}

In Figures 3-4 and 6-8, the measured $N(\mathrm{H} \mathrm{I})$ values enter the quantities plotted in both the $\mathrm{x}$ and $\mathrm{y}$-axes, raising the possibility of inaccurate or false correlations. To eliminate this possibility, we have plotted in Figure 9 the $\log [N(\mathrm{D}$ I $) / N($ Fe II $)]$ ratio vs. $\log N(\mathrm{H}$ I $)$ for all lines of sight. We include in the figure the weighted least-squares fit to all of the data points (solid line) and the fit to only the lines of sight which extend beyond the Local Bubble (dashed line). The essentially zero slope in the fit to the data beyond the Local Bubble indicates that the positive slope of the fit to the entire data set is determined by the low values of $\log [N(\mathrm{D}$ I $) / N($ Fe II $)]$ for lines of sight inside the Local Bubble. The zero slope beyond the Local Bubble indicates that on a statistical basis the deuterium and iron depletions are proportional. The scatter of the data about the two regression lines suggests different grain compositions along the various lines of sight or different processes that deplete deuterium and iron from the gas phase but that depletion is important for both species. While the scatter indicates that the depletions of $\mathrm{D}$ and Fe are not proportional on individual lines of sight, the zero slope fit to the data beyond the Local Bubble indicates that on average the rates of depletion of D and Fe appear to be proportional.

We noted in $\$ 6.3 .3$ that in regions of low shielding $(\log N(\mathrm{H} \mathrm{I})<19.0)$ from Lyman continuum radiation primarily from hot stars, that hydrogen can be partially ionized, whereas metals with second ionization potentials $>13.58 \mathrm{eV}$ will be mostly singly ionized. The second ionization potential of iron is $16.18 \mathrm{eV}$, whereas the ionization potential of neutral deuterium is the same as that of hydrogen. Thus partial ionization of deuterium could explain the decrease in $N(\mathrm{D} \mathrm{I}) / N($ Fe II $)$ at $\log N(\mathrm{H} \mathrm{I})<19.0$. It may also explain the very low ratios for the short lines of sight to 36 Oph and G191-B2B. Redfield \& Linsky (2000) found that hydrogen is half ionized in the Local Interstellar Cloud, which has a maximum column density $\log N(\mathrm{H} \mathrm{I})=18.3$. This result could simply explain the factor of two decrease in 
$N(\mathrm{D}$ I) $/ N($ Fe II) between lines of sight with large and small values of $N(\mathrm{H} \mathrm{I})$ in Figure 9.

\section{WHAT IS THE TOTAL DEUTERIUM ABUNDANCE IN THE LOCAL GALACTIC DISK?}

We propose that the most plausible explanation for the wide range in $(\mathrm{D} / \mathrm{H})_{\text {gas }}$ measurements within about $1 \mathrm{kpc}$ of the Sun is that different amounts of deuterium depletion occur in different lines of sight. We have come to this conclusion because: (i) theoretical arguments suggest that deuterium atoms can replace enough hydrogen atoms in carbonaceous grain materials to explain low values of $(\mathrm{D} / \mathrm{H})_{\text {gas }-\mathrm{LB}}$ in the undisturbed ISM, (ii) high $(\mathrm{D} / \mathrm{H})_{\text {dust }}$ ratios have been measured in interplanetary dust particles that likely came from the ISM, providing an important proof of concept for the deuterium depletion hypothesis, and (iii) the correlation of large iron and silicon depletions in the gas phase, $\mathrm{D}(\mathrm{Fe})$ and $\mathrm{D}(\mathrm{Si})$, with low values of $(\mathrm{D} / \mathrm{H})_{\text {gas }}$ strongly support the hypothesis that dust grains are an important reservoir for deuterium and that deuterium returns to the gas phase when the grains are disturbed.

The deuterium-depletion model predicts that the most likely value for the ratio of deuterium in all forms (gas plus dust) to hydrogen, $(\mathrm{D} / \mathrm{H})_{\text {total }}$, for the local region of the Galactic disk, $(\mathrm{D} / \mathrm{H})_{\mathrm{LDtot}}$, should be equal to or slightly above the highest measured $(\mathrm{D} / \mathrm{H})_{\text {gas }}$ ratios. If some deuterium remains on grains for these lines of sight, then the total amount of deuterium will slightly exceed the gas-phase value. In Figure 1 there are five lines of sight $\left(\gamma^{2} \mathrm{Vel}\right.$, Lan 23, WD 1034+001, Feige 110, and LSE 44) extending beyond the Local Bubble that have the highest values of $(\mathrm{D} / \mathrm{H})_{\text {gas }} \approx 22 \mathrm{ppm}$. These are the best candidates for inferring $(\mathrm{D} / \mathrm{H})_{\mathrm{LDtot}}$ in the local Galactic disk. The weighted mean and standard deviation of the mean for these five data points is $\left\langle(\mathrm{D} / \mathrm{H})_{\text {gas }}\right\rangle=21.7 \pm 1.7 \mathrm{ppm}$. However, the Local Bubble foreground has a known mean value of $(\mathrm{D} / \mathrm{H})_{\text {gas }}$ and extent, $\log N(\mathrm{H} \mathrm{I}) \approx 19.2$, indicating that deuterium is depleted inside the Local Bubble. We therefore subtract the Local Bubble foreground from the $(\mathrm{D} / \mathrm{H})_{\text {gas }}$ values to obtain the values of $(\mathrm{D} / \mathrm{H})_{\text {gas }-\mathrm{LB}}$ listed in Table 3. The weighted mean values for these five lines of sight is $\left\langle(\mathrm{D} / \mathrm{H})_{\text {gas }-\mathrm{LB}}\right\rangle=23.7 \pm 2.4 \mathrm{ppm}$. The line of sight to $\gamma^{2}$ Vel lies closest to the Local Bubble $(\log N(\mathrm{H} \mathrm{I})=19.710 \pm 0.026)$ and is thus most subject to the systematic errors (especially the uncertain extent of the Local Bubble in $N(\mathrm{H} \mathrm{I})$ ) associated with subtracting the Local Bubble contribution. However, this line of sight has the highest weight (58\%) in computing this mean. To minimize this potential source of systematic error, we have recomputed the mean without weighting by the inverse errors, obtaining $\left\langle(\mathrm{D} / \mathrm{H})_{\text {gas }-\mathrm{LB}}\right\rangle=23.1 \pm 2.4 \mathrm{ppm}$. Given that we have selected the data points to be used in computing the mean from a larger sample, the errors are not 
Gaussian, but we believe that our method gives the most representative value $(\mathrm{D} / \mathrm{H})_{\text {gas }-\mathrm{LB}}$ available at this time. Since even for these five lines of sight some deuterium could be in the grains, $(\mathrm{D} / \mathrm{H})_{\mathrm{LDtot}} \geq\left\langle(\mathrm{D} / \mathrm{H})_{\text {gas }-\mathrm{LB}}\right\rangle=23.1 \pm 2.4 \mathrm{ppm}$.

Even the higher estimate of the primordial D/H ratio (see Section 1$),(\mathrm{D} / \mathrm{H})_{\text {prim }}=$ $27.5_{-1.9}^{+2.4} \mathrm{ppm}$, implies a small deuterium astration (or depletion) factor for the local region of the local Galactic disk, $f_{d} \equiv(\mathrm{D} / \mathrm{H})_{\text {prim }} /(\mathrm{D} / \mathrm{H})_{\text {LDtot }} \leq\left(27.5_{-1.9}^{+2.4}\right) /(23.1 \pm 2.4) \leq 1.19_{-0.15}^{+0.16}$. For the lower estimate of $(\mathrm{D} / \mathrm{H})_{\text {prim }}=26.0_{-1.7}^{+1.9} \mathrm{ppm}, f_{d} \leq\left(26.0_{-1.7}^{+1.9}\right) /(23.1 \pm 2.4) \leq 1.12 \pm 0.14$ These small values of $f_{d}$ provide an important constraint on models of Galactic chemical evolution, which must also explain the evolution of many chemical species. The models of Chiappini, Renda, \& Matteucci (2002) (cf. Romano et al. 2003), for example, predict $f_{d} \simeq 1.5$ for a wide range of assumed $(\mathrm{D} / \mathrm{H})_{\text {prim }}$ and different rates of extra mixing in stellar interiors. These models include two epochs of infall of D-rich and metal-poor gas ${ }^{7}$ from the halo or IGM, and the models predict sensible present epoch values for ${ }^{3} \mathrm{He} / \mathrm{H},{ }^{4} \mathrm{He} / \mathrm{H}$, and ${ }^{7} \mathrm{Li} / \mathrm{H}$. By altering the assumptions regarding the rates of infall and star formation, more recent work by Romano et al. (2006) is able to reproduce current data on stellar abundance patterns in the solar neighborhood $(R=8 \mathrm{kpc})$ with astration factors $1.3<f_{d}<1.8$; the lower end of this range overlaps our determinations $f_{d}<1.19_{-0.15}^{+0.16}$ or $f_{d}<1.12 \pm 0.14$.

There are also estimates of the $\mathrm{D} / \mathrm{H}$ ratio in the protosolar cloud, $(\mathrm{D} / \mathrm{H})_{\mathrm{psc}}$, when the Galaxy was about two-thirds of its present age. Geiss \& Gloeckler (1998) have inferred $(\mathrm{D} / \mathrm{H})_{\mathrm{psc}}=21 \pm 5 \mathrm{ppm}$ based on measurements of ${ }^{3} \mathrm{He} /{ }^{4} \mathrm{He}$ in the solar wind, measurements of the same quantity in Jupiter's atmsphere, and the assumption that ${ }^{3} \mathrm{He}$ in the solar atmosphere has not undergone nuclear reactions. Analysis of Infrared Space Observatory (ISO) spectra of $\mathrm{H}_{2}, \mathrm{HD}, \mathrm{CH}_{4}$, and $\mathrm{CH}_{3} \mathrm{D}$ in Jupiter's atmosphere led Lellouch et al. (2001) to infer $(\mathrm{D} / \mathrm{H})_{\mathrm{psc}}=21 \pm 4 \mathrm{ppm}$, although there are many assumptions that go into this analysis. One cannot directly compare $(\mathrm{D} / \mathrm{H})_{\mathrm{psc}}$ with present day estimates of $(\mathrm{D} / \mathrm{H})_{\mathrm{LDtot}}$, because the Sun has moved a considerable distance from its place of birth about 4.6 Gyr ago. However, the similar values of $(\mathrm{D} / \mathrm{H})_{\mathrm{psc}}$ and $(\mathrm{D} / \mathrm{H})_{\text {LDtot }} \geq 23.1 \pm 2.4 \mathrm{ppm}$ suggest that most of the decrease in the total deuterium abundance in the ISM may have occurred as a result of nuclear reactions in stars and subsequent transfer of deuterium-depleted gas into the ISM during the first two-thirds of the age of the Galaxy.

\footnotetext{
${ }^{7}$ The infalling gas has a metal abundance $20 \%$ solar (Romano et al. 2003), which is consistent with the results for the high velocity cloud Complex C (Collins et al. 2003).
} 


\section{COULD INFALL EXPLAIN THE HIGH (D/H) gas RATIOS?}

It is fair to ask whether depletion is the only operative mechanism for changing the abundance of deuterium. Oliveira et al. (2005) have used the constancy of the $(\mathrm{O} / \mathrm{H})_{\text {gas }}$ ratios over a range of environments in the local part of the Galaxy to argue that significant changes in the $\mathrm{D} / \mathrm{H}$ ratios because of infall of lower metallicity gas are unlikely. While it is tempting to suggest significant and highly localized infall for the high $\mathrm{D} / \mathrm{H}$ sightlines, such a large infall would dramatically lower the $\mathrm{O} I$ abundances along these sightlines. A large increase in the value of $(\mathrm{D} / \mathrm{H})_{\text {gas }}$ from $15 \mathrm{ppm}$ to $22 \mathrm{ppm}$ would require a highly localized infall of gas with $(\mathrm{D} / \mathrm{H})_{\text {total }}=24 \mathrm{ppm}$ within a mixing time of about $350 \mathrm{Myr}$ (de Avillez \& Mac Low 2002). The fraction due to infall would equal more than three quarters of the mass of the gas along a given sightline. Such a large infall of D-rich, but metal-poor, gas would also lower the measured $(\mathrm{O} / \mathrm{H})_{\text {gas }}$ ratios by more than a factor of three; such low values are not observed. The constancy of $(\mathrm{O} / \mathrm{H})_{\text {gas }}$ compared to variations in $(\mathrm{D} / \mathrm{H})_{\text {gas }}$ thus argues against localized infall (Oliveira et al. 2005). Although the infall mechanism is unlikely to explain the wide range of observed $(\mathrm{D} / \mathrm{H})_{\text {gas }}$ values, we do not rule out the possibility of another, as yet unknown, mechanism for increasing the $\mathrm{D}$ abundance.

Finally, we include a note of caution. Hébrard et al. (2005) have pointed out that the $(\mathrm{D} / \mathrm{O})_{\text {gas }}$ data show variations similar to those observed for $(\mathrm{D} / \mathrm{H})_{\text {gas }}$, except that there were no high values at that time corresponding to those measured for $(\mathrm{D} / \mathrm{H})_{\text {gas. }}$. The relative uniformity of $\mathrm{O} \mathrm{I} / \mathrm{H} \mathrm{I}$ allows $\mathrm{O}$ I to be used as a proxy for $\mathrm{H} \mathrm{I}$, with the advantage that for a given line of sight, $N(\mathrm{O}$ I) is much closer to $N(\mathrm{D}$ I) than $N(\mathrm{H} \mathrm{I})$. Therefore, the D I and the O I transitions in the FUSE range have similar opacities, lowering the chances of systematic errors due to large differences in optical depth (Hébrard \& Moos 2003). A gasphase $(\mathrm{D} / \mathrm{H})_{\text {gas }}$ ratio of $23.1 \pm 2.4 \mathrm{ppm}$ and the $(\mathrm{O} / \mathrm{H})_{\text {gas }}$ ratio of $343 \pm 15 \mathrm{ppm}$ measured by Meyer et al. (1998) implies (D/O) gas $=7.02 \times 10^{-2}$, nearly twice the mean measured value of $(3.84 \pm 0.16) \times 10^{-2}$ (Hébrard \& Moos 2003). Although such high values had not previously been published, Oliveira et al. (2006) report 3 new sightlines (WD 1034+001, BD+39 3226, and TD1 32709) with high values of $(\mathrm{D} / \mathrm{O})_{\text {gas }}$, consistent with $(\mathrm{D} / \mathrm{O})_{\text {gas }} \approx 7.02 \times 10^{-2}$.

\section{CONCLUSIONS}

The main goal of the FUSE mission has been to obtain accurate measurements of $(\mathrm{D} / \mathrm{H})_{\text {gas }}$ for many sightlines in the Milky Way Galaxy and beyond in order to measure $(\mathrm{D} / \mathrm{H})_{\text {prim }}$ and to obtain constraints on Galactic chemical evolution. FUSE has now obtained the data needed for accurate measurements of $(\mathrm{D} / \mathrm{H})_{\text {gas }}$ much further out in the Galaxy and with higher precision than previous instruments. In our summary of the published 
measurements of $(\mathrm{D} / \mathrm{H})_{\text {gas }}$ obtained from FUSE, HST, Copernicus, and IMAPS, we find, following Wood et al. (2004), that the $(\mathrm{D} / \mathrm{H})_{\text {gas }}$ measurements appear to fall into three distinct groups depending on the neutral hydrogen column density $N(\mathrm{H} \mathrm{I})$ to the target star. For lines of sight with $\log N(\mathrm{H} \mathrm{I})<19.2$, that is within the Local Bubble, the mean value of $(\mathrm{D} / \mathrm{H})_{\text {gas }}$ is $15.6 \pm 0.4 \mathrm{ppm}$, where the uncertainty is the standard deviation of the mean. At large column densities, $\log N(\mathrm{H} \mathrm{I})>20.7$, we find that the five lines of sight have much lower values of $(\mathrm{D} / \mathrm{H})_{\text {gas }}$ with a mean value of $8.6 \pm 0.8 \mathrm{ppm}$. In the intermediate regime there is a factor of $4-5$ range in the high precision $(\mathrm{D} / \mathrm{H})_{\text {gas }}$ measurements with values as low as 5. $0_{-3.4}^{+2.9} \mathrm{ppm}(\theta$ Car $)$ and as high as $21.8 \pm 2.1 \mathrm{ppm}\left(\gamma^{2} \mathrm{Vel}\right)$. Since this large range has been measured from spectra obtained by several instruments, it is not an instrumental artifact, but rather an observational result that requires an explantion.

Both large changes in the local astration rates and infall of D-rich and metal-poor gas from the halo or IGM cannot explain the large range in $(\mathrm{D} / \mathrm{H})_{\text {gas }}$ without predicting large variations in the local ISM metal abundances, in particular $(\mathrm{O} / \mathrm{H})_{\text {gas }}$, which are not observed. Instead, we describe a time-dependent deuterium-depletion model in which much of the deuterium in the ISM can reside on grains under the appropriate conditions. The theoretical basis for this model is the lower zero-point energy of $\mathrm{C}-\mathrm{D}$ bonds compared to $\mathrm{C}-\mathrm{H}$ bonds. When the ISM is undisturbed for a long time, grains are very cold, and deuterium can replace hydrogen on carbonaceous grains and perhaps other types of grains. We estimate that $\mathrm{D} / \mathrm{H}$ in the grains can approach $\sim 0.2$, thereby reducing $(\mathrm{D} / \mathrm{H})_{\text {gas }}$ to $6 \mathrm{ppm}$ or lower. When a region of the ISM is shocked or the gas comes close to a hot star, the grains are partially or fully destroyed, sending deuterium atoms back into the gas phase. In this model, the value of $(\mathrm{D} / \mathrm{H})_{\text {gas }}$ for a given line of sight depends on the environment and past history of the grains.

Evidence in support of this model includes the measurement of high $\mathrm{D} / \mathrm{H}$ ratios in interplanetary carbonaceous dust grains, which are likely interstellar in origin. Since the conditions required from deuterium depletion are similar to those for depletion of metals like $\mathrm{Fe}, \mathrm{Si}$, and Ti in the ISM, the model predicts a correlation of depletions of these metals with low values of $(\mathrm{D} / \mathrm{H})_{\text {gas }}$. We present data that strongly support the correlation for Fe and $\mathrm{Si}$, and Prochaska, Tripp, \& Howk (2005) present the data in support of correlation with Ti. The model also predicts that $(\mathrm{D} / \mathrm{H})_{\text {gas }}$ should be correlated with the recent thermal and ionization history of the gas, whereas the variable astration hypothesis would not be expected to show such a connection; we find that $(\mathrm{D} / \mathrm{H})_{\text {gas }}$ has a significant positive correlation with the rotational temperature of the molecular hydrogen on the line-of-sight, further supporting the hypothesis that $(\mathrm{D} / \mathrm{H})_{\text {gas }}$ is affected by interstellar processes acting on relatively short time scales. We note that in the most recent discussion of Galactic chemical evolution models, Romano et al. (2006) conclude that "depletion of deuterium on to dust grains is the 
most likely physical mechanism proposed so far to explain the observed dispersion in the local data."

If this model is realistic, then the best estimate of the total deuterium abundance, $(\mathrm{D} / \mathrm{H})_{\text {total }}$ would be the highest measured values of $(\mathrm{D} / \mathrm{H})_{\text {gas }}$ because these would be for lines of sight with minimum depletion of deuterium. We note that there are five lines of sight in the intermediate $N(\mathrm{H} \mathrm{I})$ regime with high values of $(\mathrm{D} / \mathrm{H})_{\text {gas }}$. The weighted mean value for these four lines of sight is $21.7 \pm 1.7 \mathrm{ppm}$ (standard deviation of the mean). Since there could be some deuterium depletion even in these lines of sight, we conclude that the best estimate of the total $\mathrm{D} / \mathrm{H}$ in the local disk region of the Galaxy, after subtracting the Local Bubble foreground column densities, is $(\mathrm{D} / \mathrm{H})_{\mathrm{LDtot}} \geq 23.1 \pm 2.4 \mathrm{ppm}^{8}$. This new estimate is $\geq 58 \%$ higher than the value of 15 ppm recently used in some Galactic chemical evolution models (e.g., Romano et al. 2003), which is $(\mathrm{D} / \mathrm{H})_{\text {gas }}$ for the Local Bubble rather than $(\mathrm{D} / \mathrm{H})_{\mathrm{LDtot}}$.

Two methods for determining $(\mathrm{D} / \mathrm{H})_{\text {prim }}$ based on data from very different times in the early universe are now in agreement. Measurements of $(\mathrm{D} / \mathrm{H})_{\text {gas }}$ in five quasar absorption line systems have a mean value of $27.8_{-3.8}^{+4.4} \mathrm{ppm}$ (Kirkman et al. 2003). Analysis of data from the WMAP and other cosmic microwave experiments yield $\Omega_{b} h^{2}=0.0224$. Depending on the adopted nuclear reaction rates, $(\mathrm{D} / \mathrm{H})_{\text {prim }}=27.5_{-1.9}^{+2.4} \mathrm{ppm}$ (Cyburt, Fields, \& Olive 2003) or $26.0_{-1.7}^{+1.9} \mathrm{ppm}$ (Coc et al. 2004).

Galactic chemical evolution models attempt to explain the decrease in $\mathrm{D} / \mathrm{H}$ from the protogalaxy, for which $\mathrm{D} / \mathrm{H}$ is presumed to be the primordial value, to the present epoch where $\mathrm{D} / \mathrm{H}=(\mathrm{D} / \mathrm{H})_{\mathrm{LDtot}}$. Nuclear reactions in stars and subsequent return of D-poor and metal-rich gas by SN explosions and stellar winds will reduce deuterium in the ISM by the factor $f_{d} \equiv(\mathrm{D} / \mathrm{H})_{\text {prim }} /(\mathrm{D} / \mathrm{H})_{\text {LDtot }}$. Models of Chiappini, Renda, \& Matteucci (2002), for example, predict that $f_{d} \simeq 1.5$ for a wide range of assumptions concerning the value of $(\mathrm{D} / \mathrm{H})_{\text {prim }}$ and amount of mixing in stellar interiors. This result can be compared with our results, $f_{d} \leq\left(27.5_{-1.9}^{+2.4}\right) /(23.1 \pm 2.4) \leq 1.19_{-0.15}^{+0.16}$ or $f_{d} \leq\left(26.0_{-1.7}^{+1.9}\right) /(23.1 \pm 2.4) \leq 1.12 \pm$ 0.14 . These two results differ by $\geq 1.5 \sigma$ and $\geq 1.8 \sigma$, respectively, from the predictions of the Chiappini, Renda, \& Matteucci (2002) models. If, on the other hand, the low value

\footnotetext{
${ }^{8}$ Very recently Rogers et al. (2005) detected the hyperfine ground-state transition of atomic deuterium at $327 \mathrm{MHz}(92 \mathrm{~cm})$ in the Galactic anticenter direction. They find that $(\mathrm{D} / \mathrm{H})_{\text {gas }}=23 \pm 4 \mathrm{ppm}(1 \sigma)$ or $23_{-13}^{+15} \mathrm{ppm}(3 \sigma)$, and estimate that the column density-weighted mean distance of the emitting gas is about $2 \mathrm{kpc}$. Since the beamwidth is $14^{\circ}$, the beam averages an unknown amount of cloud and diffuse gas in and above the Galactic plane. While it is difficult to compare this new result with our line of sight measurements over shorter paths, the value of $(\mathrm{D} / \mathrm{H})_{\text {gas }}$ obtained from the hyperfine transitions of $\mathrm{D}$ and $\mathrm{H}$ is consistent with our proposed value of $(\mathrm{D} / \mathrm{H})_{\mathrm{LDtot}}$.
} 
of $(\mathrm{D} / \mathrm{H})_{\text {gas }}=8.6 \pm 0.8 \mathrm{ppm}$ for the five most distant lines of sight is representative of $(\mathrm{D} / \mathrm{H})_{\text {LDtot }}$ as Hébrard et al. (2005) proposes, then $f_{d}$ would be $\left(27.5_{-1.9}^{+2.4}\right) /(8.6 \pm 0.8)=$ $3.2_{-0.37}^{+0.41}$ or $\left(26.0_{-1.7}^{+1.9}\right) /(8.6 \pm 0.8)=3.0 \pm 0.35$, which are far larger values than the Chiappini, Renda, \& Matteucci (2002) models predict. Very recent Galactic chemical evolution models (Romano et al. 2006) can now accomodate values of $f_{d}$ as small as $\sim 1.3$, consistent with our determination $f_{d}<1.19_{-0.15}^{+0.16}$ or $1.12 \pm 0.14$, depending on the adopted value of $(\mathrm{D} / \mathrm{H})_{\text {prim }}$. However, our empirical results for $f_{d}$ are smaller than $f_{d}=1.39$, the lowest value for a model discussed by Romano et al. (2006). We suggest that new Galactic chemical evolution models should be developed to see whether smaller values of $f_{d}$ are consistent with the other empirical abundance and theoretical constraints that the models must fit.

We also call attention to the need for more high quality data to better constrain $(\mathrm{D} / \mathrm{H})_{\text {LDtot }}$ and $(\mathrm{D} / \mathrm{H})_{\text {prim. }}$. In particular, we need better quality observations of the highly saturated Lyman lines for which measurements of $N(\mathrm{H} \mathrm{I})$ may contain systematic errors of an unknown nature. Also, the assumptions underlying the Galactic chemical evolution models should be reexamined to see whether $f_{d}$ might be as small as $\sim 1.25$ without violating other constraints.

We thank the FUSE Science Team for discussions and the successful implementation of the FUSE science program. This work is supported by NASA grant S-56500-D to NIST and the University of Colorado. JLL thanks Uppsala University in Uppsala, Sweden, for hospitality where a portion of this paper was written and the International Space Science Institute in Bern, Switzerland, for support and discussions during workshops on Galactic chemical evolution. We also wish to thank the anonymous referee for his critical and insight-

ful comments and suggestions. This work was supported in part by NSF grant AST-9988126 to BTD. This work was also supported by NASA contract NAS-32985 to Johns Hopkins University.

\section{REFERENCES}

Allen, M. M., Jenkins, E. B., \& Snow, T. P. 1992, ApJS, 83, 261

Allende Prieto, C., Lambert, D. L., \& Asplund, M. 2001, ApJ, 556, L63

André, M. K. et al. 2003, ApJ, 591, 1000

Anderson, K. L., Shull, J. M., \& Tumlinson, J. 2004, Bull. AAS, 36, 772

Anders, E. \& Grevesse, N. 1989, Geochim. Cosmochim. Acta 53, 197 
Angulo, C. et al. 1999, Nuclear Physics A, 656, 3

Asplund, M., Grevesse, N., Sauval, A. J., Allende Prieto, C., \& Kiselman, D. 2004, A\&A, 417, 751

Asplund, M., Grevesse, N. \& Sauval, A. J. 2005, in Cosmic Abundances as Records of Stellar Evolution and Nucleosynthesis, ed. F. N. Bash \& T. G. Barnes (San Francisco: ASP Conf. Series), 336, 25

Bahcall, J. N., Serenelli, A. M., \& Basu, S. 2005, ApJ, 621, L85

Berghöfer, T. W. \& Breitschwerdt, D. 2002, A\&A, 390, 299

Bluhm, H., Marggraf, O., de Boer, K. S., Richter, P., \& Heber, U. 1999, A\&A, 352, 287

Boesgaard, A. M., \& Steigman, G. 1985, ARA\&A, 23, 319

Burles, S., Nollett, K. M., \& Turner, M. S. 2001, ApJ, 552, L1

Cartledge, S. I. B., Lauroesch, J. T., Meyer, D. M., \& Sofia, U. J. 2004, ApJ, 613, 1037

Chiappini, C., Renda, A., \& Matteucci, F. 2002, A\&A, 395, 789

Coc, A., Vangioni-Flan, E., Descouvemont, P., Adahchour, A., \& Angulo, C. 2004, ApJ, 600,544

Collins, J. A., Shull, J. M., \& Giroux, M. L. 2003, ApJ, 585, 336

Cox, D. P., \& Reynolds, R. J. 1987, ARA\&A, 25, 303

Cyburt, R. H., Fields, B. D., \& Olive, K. A. 2003, Physics Letters B, 567, 227

de Avillez, M. A. \& Mac Low, M.-M. 2002, ApJ, 581, 1047

Draine, B. T. 2003, ARA\&A, 41, 241

Draine, B. T. 2004, in Origin and Evolution of the Elements, ed. A. McWilliam \& M. Rauch (Cambridge: Cambridge Univ. Press), 320

Draine, B. T. 2006, in Astrophysics in the Far Ultraviolet: Five Years of Discovery with FUSE, ed: G. Sonneborn, B. G. Andersson \& Warren Moos (San Francisco: ASP), in press

Dupin, O. \& Gry, C. 1998, A\&A, 335, 661 
Dring, A. R., Linsky, J., Murthy, J., Henry, R. C., Moos, W., Vidal-Madjar, A., Audouze, J., \& Landsman, W. 1997, ApJ, 488, 760

Epstein, R. I., Lattimore, J. M., \& Schramm, D. N. 1976, Nature, 263, 198

Ferlet, R., Vidal-Madjar, A., Laurent, C., \& York, D. G. 1980, ApJ, 242, 576

Fitzpatrick, E. L., \& Spitzer Jr., L. 1994, ApJ, 427, 232

Friedman, S. D., et al. 2002, ApJS, 140, 37

Friedman, S. D., Hébrard, G., Tripp, T. M., Chayer, P., \& Sembach, K. R. 2006, ApJ, 638, 847

Frisch, P. C., et al. 1999, ApJ, 525, 492

Frisch, P. C. \& Slavin, J. D. 2003, ApJ, 594, 844

Geiss, J. \& Gloeckler, G. 1998, Space Sci. Rev., 84, 239

Geiss, J., Gloeckler, G., \& Charbonnel, C. 2002, ApJ, 578, 862

Gillmon, K., Shull, J. M., Tumlinson, J., \& Danforth, C. 2006, ApJ, 636, 891

Gry, C., York, D. G., \& Vidal-Madjar, A. 1985, ApJ, 296, 593

Hébrard, G., Mallouris, C., Koester, D., Lemoine, M., Vidal-Madjar, A., \& York, D. 1999, A\&A, 350, 643

Hébrard, G., et al. 2002, ApJS, 140, 103

Hébrard, G., Tripp, T. M., Chayer, P., Friedman, S. D., Dupuis, J., Sonnentrucker, P., Williger, G. M., \& Moos, H. W. 2005, ApJ, 635, 1136

Hébrard, G. \& Moos, H. W. 2003, ApJ, 599, 297

Hoopes, C. G., Sembach, K. R., Hébrard, G., Moos, H. W., \& Knauth, D. C. 2003, ApJ, 586,1094

Howk, J. C., Savage, B. D., \& Fabian, D. 1999, ApJ, 525, 253

Howk, J. C., Sembach, K. R., Roth, K. C., \& Kruk, J. W. 2000, ApJ, 544, 867

Jenkins, E. B. 1996, in Cosmic Abundances, ed. S. S. Holt \& G. Sonneborn (San Francisco: ASP Conf. Series), 99, 90 
Jenkins, E. B. 2004, in Origin and Evolution of the Elements, ed. A. McWilliam \& M. Rauch (Cambridge: Cambridge Univ. Press), 336

Jenkins, E. B., Gry, C., \& Dupin, O. 2000a, A\&A, 354, 253

Jenkins, E. B., Wozniak, P. R., Sofia, U. J., Sonneborn, G., \& Tripp, T. M. 2000b, ApJ, 538,275

Jenkins, E. B., Tripp, T. M., Wozniak, P. R., Sofia, U. J., \& Sonneborn, G. 1999, ApJ, 520, 182

Jenkins, E. B., Savage, B. D., \& Spitzer Jr., L. 1986, ApJ, 301, 355

Jones, A. P. 2004, JGR, 105, A5, 10257

Jones, A. P., Tielens, A. G. G. M., Hollenbach, D. J., \& McKee, C. F. 1994, ApJ, 433, 797

Jura, M. 1982, in Advances in UV Astronomy: 4 Years of IUE Research, ed. Y. Kondo, J. M. Mead, \& Chapman, R. D. (NASA CP 2238; Greenbelt MD: NASA), 54

Keller, L. P., Messenger, S., \& Bradley, J. P. 2000, J. Geophys. Res., 105, 10397

Kirkman, D., Tytler, D., Suzuki, N., O’Meara, J., \& Lubin, D. 2003, ApJS, 149, 1

Kruk, J. W., et al. 2002, ApJS, 140, 19

Lallement, R., Welsh, B. Y., Vergely, J. L., Crifo, F., \& Sfeir, D. 2003, A\&A, 411, 447

Laurent, C., Vidal-Madjar, A., \& York, D. G. 1979, ApJ, 229, 923

Lehner, N., Gry, C., Sembach, K. R., Hébrard, G., Chayer, P., Moos, H. W., Howk, J. C., \& Désert, J. -M. 2002, ApJS, 140, 81

Lehner, N., Jenkins, E. B., Gry, C., Moos, H. W., Chayer, P., \& Lacour, S. 2003, ApJ, 595, 858

Lehner, N., Wakker, B. P., \& Savage, B. D. 2004, ApJ, 615, 767

Lellouch, E., Bézard, B., Fouchet, T., Feuchtgruber, H., Encrenaz, T., \& de Graauw, T. 2001, A\&A, 670, 610

Lemoine, M., et al. 2002, ApJS, 140, 67

Li, A., \& Draine, B. T. 2001, ApJ, 554, 778 
Linsky, J. L. 1998, Space Sci. Rev. 84, 285

Linsky, J. L. 2003, Space Sci. Rev. 106, 49

Linsky, J. L. 2006, in Astrophysics in the Far Ultraviolet: Five Years of Discovery with FUSE, ed. G. Sonneborn, B. G. Andersson \& Warren Moos (San Francisco: ASP), in press

Linsky, J. L., Diplas, A., Wood, B. E., Brown, A., Ayres, T. R., \& Savage, B. D. 1995, ApJ, 451,335

Lockman, F. J. 2002, ApJ, 580, L47

Lockman, F. J., Jahoda, K., \& McCammon, D. 1986, ApJ, 302, 432

Lyu, C. H. \& Bruhweiler, F. C. 1996, ApJ, 459, 216

Markwick, A. J., Charnley, S. B., \& Millar, T. J. 2001, A\&A, 376, 1054

Maíz-Apellániz, J. 2001, ApJ, 560, L83

Mauche, C. W., Raymond, J. C., \& Cordova, F. A. 1988, ApJ, 335, 829

Meléndez, J. 2004, ApJ, 615, 1042

Meyer, D. M. 2001, in Gaseous Matter in Galaxies and Intergalactic Space, ed. R. Ferlet et al. (Paris: Editions Frontiérs), 135

Meyer, D. M., Jura, M., \& Cardelli, J. A. 1998, ApJ, 493, 222

Moos, H. W. et al. 2000, ApJ, 538, L1

Moos, H. W. et al. 2002, ApJS, 140, 3

Morton, D. C. 1978, ApJ, 222, 863

Morton, D. C. 2003, ApJS, 149, 205

Nollett, K. M., \& Burles, S. 2000, Phys. Rev. D, 61, 123505

Oliveira, C. M., Dupuis, J., Chayer, P., \& Moos, H. W. 2005, ApJ, 625, 232

Oliveira, C. M., Hébrard, G., Howk, J. C., Kruk, J. W., Chayer, P., \& Moos, H. W. 2003, ApJ, 587, 235 
Oliveira, C. M., Moos, H. W., Chayer, P., \& Kruk, J. W. 2006, ApJ, accepted (astro$\mathrm{ph} / 0601114)$.

Parise, B., et al. 2002, A\&A, 393, L49

Parise, B., Castets, A., Herbst, E., Caux, E., Ceccarelli, C., Mukhopadhyay, L., \& Tielens, A. G. G. M. 2004, A\&A, 416, 159

Peeters, E., Allamandola, L. J., Bauschlicher Jr., C. W., Hudgins, D. M., Sandford, S. A., \& Tielens, A. G. G. M. 2004, ApJ, 604, 252

Pendleton, Y. J., \& Allamandola, L. J. 2002, ApJS, 138, 75

Piskunov, N., Wood, B. E., Linsky, J. L., Dempsey, R. C., \& Ayres, T. R. 1997, ApJ, 474, 315

Prochaska, J. X., Tripp, T. M., \& Howk, J. C. 2005, ApJ, 620, L39

Redfield, S., \& Linsky, J. L. 2000, ApJ, 534, 825

Redfield, S., \& Linsky, J. L. 2002, ApJS, 139, 439

Redfield, S., \& Linsky, J. L. 2004a, ApJ, 602, 776

Redfield, S., \& Linsky, J. L. 2004b, ApJ, 613, 1004

Reynoso, E. M., \& Dubner, G. M. 1997, A\&AS, 123, 31

Rogers, A. E. E., Dudevoir, K. A., Carter, J. C., Fanous, B. J., Kratzenberg, E., \& Bania, T. M. 2005, ApJ, 630, L41

Romano, D., Tosi, M., Matteucci, F., \& Chiappini, C. 2003, MNRAS, 346, 295

Romano, D., Tosi, M., Chiappini, C., \& Matteucci, F. 2006, MNRAS, accepted (astro$\mathrm{ph} / 0603190)$

Sahnow, D. J. et al. 2000, ApJ, 538, L7

Sánchez, A. G., Baugh, C. M., Percival, W. J., Peacock, J. A., Padilla, N. D., Cole, S., Frenk, C. S., Norberg, P. 2006, MNRAS, 366, 189

Savage, B. D. \& Sembach, K. R. 1996, ARA\&A, 34, 279

Savage, B. D., Drake, J. F., Bohlin, R. C., \& Budich, W. 1977, ApJ, 216, 291 
Seaton, M. J. \& Badnell, N. R. 2004, MNRAS, 354, 457

Sembach, K. R., \& Savage, B. D. 1996, ApJ, 457, 211

Sembach, K. R., et al. 2004, ApJS, 150, 387

Sfeir, D. M., Lallement, R., Crifo, F., \& Welsh, B. Y. 1999, A\&A, 346, 785

Shull, J. M., Anderson, K. L., Tumlinson, J., et al. 2005, ApJ, submitted

Shull, J. M. \& York, D. G. 1977, ApJ, 211, 803

Sofia, U. J., Cardelli, J. A., \& Savage, B. D. 1994, ApJ, 430, 650

Sonneborn, G., Tripp, T. M., Ferlet, R., Jenkins, E. B., Sofia, U. J., Vidal-Madjar, A., \& Wozniak, P. R. 2000, ApJ, 545, 277

Sonneborn, G., et al. 2002, ApJS, 140, 51

Spergel, D. N., et al. 2003, ApJS, 148, 175

Spitzer, L. Jr., Cochran, W. D., \& Hirshfeld, A. 1974, ApJS, 28, 373

Spitzer, L. Jr., \& Fitzpatrick, E. L. 1993, ApJ, 409, 299

Spitzer, L. Jr. \& Jenkins, E. B. 1975, ARA\&A, 13, 133

Tielens, A. G. G. M. 1983, A\&A, 119, 177

Turner, B. E. 1990, ApJ, 362, L29

van der Tak, F. F. S., Schilke, P., Müller, H. S. P., Lis, D. C., Phillips, T. G., Gerin, M., \& Roueff, E. 2002, A\&A, 388, L53

van Steenberg, M. E. \& Shull, J. M. 1988, ApJS, 67, 225

Vennes, S., Polomski, E. F., Lanz, T., Thorstensen, J. R., Chayer, P., \& Gull, T. R. 2000, ApJ, 544, 423

Vidal-Madjar, A., Laurent, C., Bonnett, R. M., \& York, D. G. 1977, ApJ, 211, 91

Wakker, B. P., \& Mathis, J. S. 2000, ApJ, 544, L107

Welty, D. E., Hobbs, L. M., \& Kulkarni, V. P. 1994, ApJ, 436, 152 
Williger, G. M., Oliveira, C., Hébrard, G., Dupuis, J., Dreizler, S., \& Moos, H. W. 2005, ApJ, 625, 210

Wilson, T. L. \& Rood, R. T. 1994, ARA\&A, 32, 191

Wood, B. E., Alexander, W. R., \& Linsky, J. L. 1996, ApJ, 470, 1157

Wood, B. E., Linsky, J. L., \& Zank, G. P. 2000, ApJ, 537, 304

Wood, B. E., Linsky, J. L., Hébrard, G., Vidal-Madjar, A., Lemoine, M., Moos, H. W., Sembach, K. R., \& Jenkins, E. B. 2002, ApJS, 140, 91

Wood, B. E., Linsky, J. L., Hébrard, G., Williger, G. M., Moos, H. W., \& Blair, W. P. 2004, ApJ, 609, 838

Yan, H., Lazarian, A., \& Draine, B. T. 2004, ApJ, 616, 895

York, D. G. 1983, ApJ, 264, 172

York, D. G. \& Rogerson, J. B. 1976, ApJ, 203, 378 
Table 1. PRIMORDIAL D/H: MEASURED AND INFERRED ${ }^{\mathrm{a}}$

\begin{tabular}{lllll}
\hline \multicolumn{1}{c}{ References } & \multicolumn{1}{c}{$\Omega_{B} h^{2}$} & \multicolumn{1}{c}{$\eta_{10}$} & $\begin{array}{c}(\mathrm{D} / \mathrm{H})_{\text {prim }} \\
(\mathrm{ppm})\end{array}$ & \multicolumn{1}{c}{ Data Used $^{\mathrm{b}}$} \\
& & & & \\
\hline Kirkman et al. (2003) & $0.0214 \pm 0.0020$ & $5.9 \pm 0.5$ & $27.8_{-3.8}^{+4.4}$ & 5 quasar absorption line systems \\
Spergel et al. (2003) & $0.0224 \pm 0.0009$ & $6.1_{-0.2}^{+0.3}$ & $26.2_{-2.0}^{+1.8}$ & CMB, 2dFGRS, and L $\alpha$ forest data \\
Sánchez et al. (2006) & $0.0225 \pm 0.0010$ & $6.15 \pm 0.27$ & $25.9_{-1.7}^{+1.9}$ & CMB, 2dFGRS, 6 parameter model \\
Cyburt, Fields, \& Olive (2003) & $0.0224 \pm 0.0009$ & $6.14 \pm 0.25$ & $27.5_{-1.9}^{+2.4}$ & CMB, new nuclear reaction rates \\
Coc et al. (2004) & $0.0224 \pm 0.0009$ & $6.14 \pm 0.25$ & $26.0_{-1.7}^{+1.9}$ & WMAP, SBBN, new reaction rates \\
\hline
\end{tabular}

${ }^{\mathrm{a}}$ Quoted uncertainties are $\pm 1 \sigma$.

${ }^{\mathrm{b}} \mathrm{CMB}$ refers to the cosmic microwave background data consisting primarily of data from the Wilkinson Microwave Anisotropy Probe (WMAP) but also finer scale CMB experiments. 2dFGRS refers to the power spectrum of galaxy clustering measured from the final two-degree field galaxy redshift survey (Sánchez et al. 2006). SBBN refers to standard big band nucleosynthesis.

${ }^{\mathrm{c}}$ Nuclear reaction rates of Angulo et al. (1999).

${ }^{\mathrm{d}}$ Nuclear reaction rates cited in Coc et al. (2004). 
Table 2. COMPILATION OF COLUMN DENSITY MEASUREMENTS

\begin{tabular}{|c|c|c|c|c|c|c|c|c|c|c|}
\hline Target & $\begin{array}{c}1 \\
(\mathrm{deg})\end{array}$ & $\begin{array}{c}\mathrm{b} \\
(\mathrm{deg})\end{array}$ & $\begin{array}{c}\mathrm{d} \\
(\mathrm{pc})\end{array}$ & $\log N(\mathrm{H} \mathrm{I})^{\mathrm{a}}$ & $\log N(\mathrm{D} \mathrm{I})^{\mathrm{a}}$ & Satellite & Flag ${ }^{b}$ & $\log N(\mathrm{Fe} \mathrm{II})^{\mathrm{a}}$ & $\log N(\mathrm{Si} \mathrm{II})^{\mathrm{a}}$ & Refs. \\
\hline Sirius $^{h}$ & 227 & -09 & $2.64 \pm 0.02$ & $17.60_{-0.12}^{+0.14}$ & $12.81 \pm 0.09$ & HST & $\mathrm{LBg}$ & $11.94_{-0.016}^{+0.014}$ & $12.48_{-0.08}^{+0.12}$ & 42 \\
\hline $36 \mathrm{Oph}$ & 358 & +07 & $5.99 \pm 0.04$ & $17.85 \pm 0.07$ & $13.025 \pm 0.01$ & HST & $\mathrm{LBg}$ & $12.65 \pm 0.25$ & & 4,27 \\
\hline$\epsilon$ Eri & 227 & -48 & $3.22 \pm 0.01$ & $17.875 \pm 0.035$ & $13.03 \pm 0.03$ & HST & $\mathrm{LBg}$ & $12.26 \pm 0.10$ & $12.76 \pm 0.14$ & $1,27,28$ \\
\hline 31 Com & 115 & +89 & $94 \pm 8$ & $17.884 \pm 0.03$ & $13.19 \pm 0.025$ & HST & $\mathrm{LBg}$ & $12.55 \pm 0.10$ & & $1,27,28$ \\
\hline HZ 43 & 54 & +84 & $68 \pm 13$ & $17.93 \pm 0.03$ & $13.15 \pm 0.02$ & FUSE & LBg & $12.17 \pm 0.02$ & $12.69 \pm 0.06$ & $8,27,28$ \\
\hline$\epsilon$ Ind & 336 & -48 & $3.63 \pm 0.01$ & $18.00 \pm 0.05$ & $13.20 \pm 0.027$ & HST & $\mathrm{LBg}$ & & & 3 \\
\hline Procyon & 214 & +13 & $3.50 \pm 0.01$ & $18.06 \pm 0.05$ & $13.26 \pm 0.027$ & HST & $\mathrm{LBg}$ & $12.27 \pm 0.02$ & & 2,28 \\
\hline$\beta$ Cas & 118 & -03 & $16.7 \pm 0.1$ & $18.132 \pm 0.025$ & $13.36 \pm 0.03$ & HST & LBg & $12.36 \pm 0.10$ & & 1,27 \\
\hline HR 1099 & 185 & -41 & $29.0 \pm 0.7$ & $18.131 \pm 0.020$ & $13.295 \pm 0.023$ & HST & $\mathrm{LBg}$ & & & $5,27,28$ \\
\hline G191-B2B & 156 & +07 & $69 \pm 15$ & $18.18 \pm 0.09$ & $13.40 \pm 0.035$ & FUSE & $\mathrm{LBg}$ & $13.05 \pm 0.02$ & $12.55_{-0.14}^{+0.15}$ & $9,27,28$ \\
\hline$\beta \mathrm{CMa}^{\mathrm{c}}$ & 226 & -14 & $153 \pm 15$ & $18.20_{-0.20}^{+0.14}$ & $\geq 13.40$ & Copernicus & $\mathrm{LBg}$ & $13.04 \pm 0.04$ & $13.745 \pm 0.04$ & 16,29 \\
\hline$\sigma \mathrm{Gem}$ & 191 & +23 & $37 \pm 1$ & $18.201 \pm 0.037$ & $\overline{13} .34 \pm 0.05$ & HST & $\mathrm{LBg}$ & & & 1 \\
\hline Capella & 163 & +05 & $12.9 \pm 0.1$ & $18.239 \pm 0.035$ & $13.44 \pm 0.02$ & HST & LBg & $12.49 \pm 0.02$ & $13.00 \pm 0.05$ & $2,27,28$ \\
\hline$\beta$ Gem & 192 & +23 & $10.34 \pm 0.09$ & $18.261 \pm 0.037$ & $13.43 \pm 0.05$ & HST & $\mathrm{LBg}$ & $12.42 \pm 0.03$ & & 1,27 \\
\hline$\alpha$ Tri & 139 & -31 & $19.7 \pm 0.3$ & $18.327 \pm 0.035$ & $13.45 \pm 0.05$ & HST & $\mathrm{LBg}$ & $12.65 \pm 0.20$ & & 1,27 \\
\hline$\beta$ Cet & 111 & -81 & $29.4 \pm 0.7$ & $18.36 \pm 0.05$ & $13.70_{-0.11}^{+0.08}$ & HST & $\mathrm{LBg}$ & & $14.17 \pm 0.41$ & 23,28 \\
\hline$\lambda$ And & 110 & -15 & $25.8 \pm 0.5$ & $18.45 \pm 0.075$ & $13.68 \pm 0.10$ & HST & $\mathrm{LBg}$ & & & 3 \\
\hline Feige 24 & 166 & -50 & $74 \pm 20$ & $18.47 \pm 0.03$ & $13.58 \pm 0.14$ & HST & $\mathrm{LBg}$ & & $13.54 \pm 0.07$ & 10 \\
\hline WD 0621-376 & 245 & -21 & $78 \pm 23$ & $18.70 \pm 0.15$ & $13.85 \pm 0.045$ & FUSE & $\mathrm{LBg}$ & $12.94_{-0.16}^{+0.14}$ & & 11 \\
\hline WD 2211-495 & 346 & -53 & $53 \pm 16$ & $18.76 \pm 0.15$ & $13.94 \pm 0.05$ & FUSE & $\mathrm{LBg}$ & $13.25 \pm 0.075$ & $14.00 \pm 0.08$ & 7,30 \\
\hline WD 1634-573 & 330 & -07 & $37 \pm 3$ & $18.85 \pm 0.06$ & $14.05 \pm 0.025$ & FUSE & $\mathrm{LBg}$ & $13.08 \pm 0.065$ & $13.76^{+0.13}$ & 6,30 \\
\hline$\alpha$ Vir & 316 & +51 & $80 \pm 6$ & $19.0 \pm 0.1$ & $14.2_{-0.1}^{+0.2}$ & Copernicus & $\mathrm{LBg}$ & $13.16_{-0.08}^{+0.01}$ & $14.16_{-0.08}^{+0.17}$ & 13,32 \\
\hline GD 246 & 87 & -45 & $79 \pm 24$ & $19.11 \pm 0.025$ & $14.29 \pm 0.045$ & FUSE & $\mathrm{LBg}$ & $13.30 \pm 0.05$ & $14.07_{-0.04}^{+0.08}$ & 12 \\
\hline$\lambda \mathrm{Sco}^{\mathrm{d}}$ & 352 & -02 & $216 \pm 42$ & $19.23 \pm 0.03$ & $14.11^{+0.09}$ & Copernicus & INT & $13.04 \pm 0.04$ & $13.42 \pm 0.04$ & 19 \\
\hline$\beta$ Cen & 312 & +01 & $161 \pm 15$ & $19.63 \pm 0.10$ & $14.7 \pm 0.2$ & IUE, Copernicus & INT & $13.92_{-0.04}^{+0.05}$ & $14.59 \pm 0.01$ & 13,32 \\
\hline$\gamma^{2} \mathrm{Vel}$ & 263 & -08 & $258 \pm 35$ & $19.710 \pm 0.026$ & $15.05 \pm 0.03$ & IMAPS & INT & $13.95 \pm 0.11$ & $15.03 \pm 0.04$ & 20,26 \\
\hline$\alpha \mathrm{Cru}$ & 300 & +00 & $98 \pm 6$ & $19.85_{-0.10}^{+0.07}$ & $14.95 \pm 0.05$ & Copernicus & INT & $14.00 \pm 0.10$ & & 13,37 \\
\hline $\mathrm{BD}+28^{\circ} 4211$ & 82 & -19 & $104 \pm 18$ & $19.846 \pm 0.018$ & $14.99 \pm 0.025$ & FUSE & INT & $14.10 \pm 0.10$ & & $14,38,39$ \\
\hline Lan 23 & 108 & -01 & $122 \pm 37$ & $19.89_{-0.04}^{+0.25}$ & $15.23 \pm 0.065$ & FUSE, EUVE & INT & $14.03 \pm 0.065$ & & 12,41 \\
\hline$\mu \mathrm{Col}^{\mathrm{e}}$ & 237 & -27 & $400_{-70}^{+100}$ & $19.86 \pm 0.015$ & $14.7_{-0.1}^{+0.3}$ & HST, Coper. & INT & $14.13 \pm 0.02$ & $15.10 \pm 0.02$ & 13,35 \\
\hline$\zeta$ Pup & 256 & -05 & $429 \pm 94$ & $19.963 \pm 0.026$ & $15.11 \pm 0.06$ & IMAPS & INT & $14.13_{-0.07}^{+0.17}$ & $15.07_{-0.02}^{+0.07}$ & 20,32 \\
\hline TD1 32709 & 233 & +28 & $520 \pm 90$ & $20.03 \pm 0.10$ & $15.30 \pm 0.05$ & FUSE & INT & $13.95 \pm 0.10$ & & 44 \\
\hline WD1 $1034+001$ & 248 & +48 & $155_{-43}^{+58}$ & $20.07 \pm 0.07$ & $15.40 \pm 0.07$ & FUSE & INT & $14.10 \pm 0.10$ & & 44 \\
\hline
\end{tabular}


Table 2-Continued

\begin{tabular}{|c|c|c|c|c|c|c|c|c|c|c|}
\hline Target & $\begin{array}{c}1 \\
(\mathrm{deg})\end{array}$ & $\begin{array}{c}\mathrm{b} \\
(\mathrm{deg})\end{array}$ & $\begin{array}{c}\mathrm{d} \\
(\mathrm{pc})\end{array}$ & $\log N(\mathrm{H} \mathrm{I})^{\mathrm{a}}$ & $\log N(\mathrm{D} \mathrm{I})^{\mathrm{a}}$ & Satellite & Flagb & $\log N(\mathrm{Fe} \text { II })^{\mathrm{a}}$ & $\log N(\mathrm{Si} \mathrm{III})^{\mathrm{a}}$ & Refs. \\
\hline $\mathrm{BD}+39^{\circ} 3226$ & 65 & +29 & $290_{-70}^{+140}$ & $20.08 \pm 0.09$ & $15.15 \pm 0.05$ & FUSE & INT & $14.15 \pm 0.07$ & $14.80 \pm 0.20$ & 31,44 \\
\hline Feige 110 & 74 & -59 & $179_{-67}^{+265}$ & $20.14_{-0.10}^{+0.065}$ & $15.47 \pm 0.03$ & FUSE, IUE & INT & & & $17,38,39$ \\
\hline$\gamma$ Cas & 124 & -02 & $188 \pm 20$ & $20.16_{-0.10}^{+0.10}$ & $15.15_{-0.05}^{+0.04}$ & Copernicus & INT & $14.08_{-0.17}^{+0.11}$ & & 18,37 \\
\hline$\iota$ Ori & 210 & -20 & $407 \pm 127$ & $20.15_{-0.07}^{+0.10}$ & $\begin{array}{l}10.10_{-0.05}^{+0.05} \\
15.30_{-0.05}^{+0.04}\end{array}$ & Copernicus & INT & $\begin{array}{l}14.00-0.17 \\
14.20_{-0.15}^{+0.20}\end{array}$ & $15.16_{-0.03}^{+0.02}$ & $\begin{array}{l}10,31 \\
22,32,37,38\end{array}$ \\
\hline$\delta$ Ori & 204 & -18 & $281 \pm 65$ & $20.19 \pm 0.03$ & $15.06_{-0.04}^{+0.06}$ & IMAPS & INT & $14.08 \pm 0.03$ & & $21,32,40$ \\
\hline$\theta \operatorname{Car}^{\mathrm{f}}$ & 290 & -05 & $135 \pm 9$ & $20.28 \pm 0.10$ & $14.98_{-0.21}^{+0.04}$ & Copernicus & INT & $14.19 \pm 0.03$ & $14.38_{-0.08}^{+0.25}$ & 15 \\
\hline$\epsilon$ Ori & 205 & -17 & $412 \pm 154$ & $20.45_{-0.10}^{+0.08}$ & $15.25 \pm 0.05$ & Copernicus & INT & $14.20 \pm 0.10$ & $15.02_{-0.12}^{+0.08}$ & $22,32,37$ \\
\hline PG $0038+199^{9}$ & 120 & -43 & $297_{-104}^{+164}$ & $20.48 \pm 0.04$ & $15.76 \pm 0.04$ & FUSE & INT & $14.42_{-0.02}^{+0.03}$ & & 25 \\
\hline LSE 44 & 313 & +13 & $554 \pm 66$ & $20.52_{-0.18}^{+0.10}$ & $15.87 \pm 0.04$ & FUSE, IUE & INT & & & 43 \\
\hline JL 9 & 323 & -27 & $590 \pm 160$ & $20.78 \pm 0.05$ & $15.78 \pm 0.06$ & FUSE & LDg & $14.69 \pm 0.085$ & & 23 \\
\hline HD 195965 & 86 & +05 & $794 \pm 200$ & $20.95 \pm 0.025$ & $15.88 \pm 0.07$ & FUSE & $\mathrm{LDg}$ & $14.81 \pm 0.01$ & & 24,38 \\
\hline LSS 1274 & 277 & -05 & $580 \pm 100$ & $20.98 \pm 0.04$ & $15.86 \pm 0.09$ & FUSE & $\mathrm{LDg}$ & $14.81 \pm 0.075$ & & 23,39 \\
\hline HD 191877 & 62 & -06 & $2200 \pm 550$ & $21.05 \pm 0.05$ & $15.94_{-0.06}^{+0.11}$ & FUSE & LDg & $14.95 \pm 0.02$ & & 24,38 \\
\hline HD $90087^{g}$ & 285 & -02 & $2740 \pm 800$ & $21.22 \pm 0.05$ & $16.16 \pm 0.06$ & FUSE & $\mathrm{LDg}$ & $15.22 \pm 0.02$ & & 41 \\
\hline
\end{tabular}

${ }^{\text {a }}$ Quoted uncertainties assumed to be $1 \sigma$ (see text). $N(\mathrm{H} \mathrm{I})$ is the most recent published value. $N(\mathrm{D}$ I) is not listed when not in original paper. $N($ Fe II) and $N(\mathrm{Si}$ II) have been computed using recent, consistent f-values (see $\S 6.1$ ).

${ }^{\mathrm{b}}$ Indicates which lines of sight are used to compute the gas-phase Local Bubble (LBg) and gas-phase local disk (LDg) D/H values described in the text. INT indicates the intermediate regime, $\log \mathrm{N}(\mathrm{H} \mathrm{I})=19.2-20.7$.

${ }^{\mathrm{c}} \mathrm{D}(\mathrm{Fe})$ and $\mathrm{D}(\mathrm{Si})$ refer to velocity Component $\mathrm{C}$ (Dupin \& Gry 1998). N(H I) and N(D I) mostly in Component C.

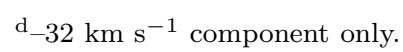

${ }^{e} \mathrm{D}(\mathrm{Fe})$ and $\mathrm{D}(\mathrm{Si})$ refer to Component 1 (Howk et al. 1999), which is also called Component A (Shull \& York 1977).

${ }^{\mathrm{f}}$ Abundances and depletions refer only to the H I component (Allen et al. 1992).

${ }_{\mathrm{g}} \mathrm{N}(\mathrm{H} \mathrm{I})$ includes $2 \mathrm{~N}\left(\mathrm{H}_{2}\right)$ and $(\mathrm{D} / \mathrm{H})_{\text {gas }}=[\mathrm{N}(\mathrm{D} \mathrm{I})+\mathrm{N}(\mathrm{HD})] /\left[\mathrm{N}(\mathrm{H} \mathrm{I})+2 \mathrm{~N}\left(\mathrm{H}_{2}\right)\right]$.

${ }^{\mathrm{h}}$ The Local Interstellar Cloud component only. For the $\mathrm{BC}$ component $(\mathrm{D} / \mathrm{H})_{\text {gas }}=5_{-5}^{+11} \mathrm{ppm}$.

References. - (1) Dring et al. 1997. (2) Linsky et al. 1995. (3) Wood et al. 1996. (4) Wood et al. 2000. (5) Piskunov et al. 1997. (6) Wood et al. 2002. (7) Hébrard et al. 2002. (8) Kruk et al. 2002. (9) Lemoine et al. 2002. (10) Vennes et al. 2000. (11) Lehner et al. 2002. (12) Oliveira et al. 2003. (13) York \& Rogerson 1976. (14) Sonneborn et al. 2002. (15) Allen et al. 1992. (16) Gry et al. 1985. (17) Friedman et al. 2002. (18) Ferlet 
et al. 1980. (19) York 1983. (20) Sonneborn et al. 2000. (21) Jenkins et al. 1999. (22) Laurent et al. 1979. (23) Wood et al. 2004. (24) Hoopes et al. 2003. (25) Williger et al. 2005. (26) Fitzpatrick \& Spitzer 1994. (27) Redfield \& Linsky 2002. (28) Redfield \& Linsky 2004a. (29) Dupin \& Gry 1998. (30) Lehner et al. 2003. (31) Bluhm et al. 1999. (32) van Steenberg \& Shull 1988. (33) Mauche, Raymond, \& Cordova 1988. (34) Spitze \& Fitzpatrick 1993. (35) Howk, Savage, \& Fabian 1999. (36) Morton 1978. (37) Jenkins, Savage, \& Spitzer 1986. (38) Prochaska et al. 2005. (39) Hébrard \& Moos 2003. (40) Howk et al. 2000. (41) Hébrard et al. 2005. (42) Hébrard et al. 1999. (43) Friedman et al. 2006. (44) Oliveira et al. 2006. 
Table 3. COMPILATION OF D/H RATIOS AND METAL DEPLETIONS

\begin{tabular}{|c|c|c|c|c|c|c|c|c|}
\hline Target & $\begin{array}{c}1 \\
(\operatorname{deg})\end{array}$ & $\begin{array}{c}\mathrm{b} \\
(\mathrm{deg})\end{array}$ & $\begin{array}{c}\mathrm{d} \\
(\mathrm{pc})\end{array}$ & $\log N(\mathrm{H} \mathrm{I})^{\mathrm{a}}$ & $\begin{array}{l}(\mathrm{D} / \mathrm{H})_{\operatorname{gas}^{\mathrm{a}}} \\
(\mathrm{ppm})\end{array}$ & $\begin{array}{l}(\mathrm{D} / \mathrm{H})_{\operatorname{gas}-\mathrm{LB}^{\mathrm{a}}} \\
\quad(\mathrm{ppm})\end{array}$ & $\mathrm{D}(\mathrm{Fe})^{\mathrm{a}}$ & $\mathrm{D}(\mathrm{Si})^{\mathrm{a}}$ \\
\hline Sirius $^{\text {h }}$ & 227 & -09 & $2.64 \pm 0.02$ & $17.60_{-0.12}^{+0.14}$ & $16.2_{-7.2}^{+6.4}$ & & $-1.11_{-0.14}^{+0.12}$ & $-0.63 \pm 0.16$ \\
\hline $36 \mathrm{Oph}$ & 358 & +07 & $5.99 \pm 0.04$ & $17.850 \pm 0.075$ & $15 \pm 2.5$ & & $-0.65 \pm 0.27$ & \\
\hline$\epsilon$ Eri & 227 & -48 & $3.22 \pm 0.01$ & $17.875 \pm 0.035$ & $14.3 \pm 1.6$ & & $-1.07 \pm 0.10$ & $-0.63 \pm 0.14$ \\
\hline $31 \mathrm{Com}$ & 115 & +89 & $94 \pm 8$ & $17.884 \pm 0.03$ & $20.2 \pm 1.9$ & & $-0.78 \pm 0.10$ & \\
\hline HZ 43 & 54 & +84 & $68 \pm 13$ & $17.93 \pm 0.03$ & $16.6 \pm 1.4$ & & $-1.21 \pm 0.04$ & $-0.75 \pm 0.07$ \\
\hline$\epsilon$ Ind & 336 & -48 & $3.63 \pm 0.01$ & $18.00 \pm 0.05$ & $16 \pm 2$ & & & \\
\hline Procyon & 214 & +13 & $3.50 \pm 0.01$ & $18.06 \pm 0.05$ & $16 \pm 2$ & & $-1.24 \pm 0.05$ & \\
\hline$\beta$ Cas & 118 & -03 & $16.7 \pm 0.1$ & $18.132 \pm 0.025$ & $16.9 \pm 1.6$ & & $-1.22 \pm 0.10$ & \\
\hline HR 1099 & 185 & -41 & $29.0 \pm 0.7$ & $18.131 \pm 0.020$ & $14.6 \pm 1.0$ & & & \\
\hline G191-B2B & 156 & +07 & $69 \pm 15$ & $18.18 \pm 0.09$ & $16.6 \pm 4.1$ & & $-0.58 \pm 0.09$ & $-1.14_{-0.16}^{+0.17}$ \\
\hline$\beta \mathrm{CMa}^{\mathrm{c}}$ & 226 & -14 & $153 \pm 15$ & $18.20_{-0.20}^{+0.14}$ & $\geq 16$ & & $-0.61_{-0.14}^{+0.20}$ & $\begin{array}{r}+0.04_{-0.14}^{+0.20} \\
\end{array}$ \\
\hline$\sigma$ Gem & 191 & +23 & $37 \pm 1$ & $18.201 \pm 0.037$ & $13.8 \pm 2.1$ & & & \\
\hline Capella & 163 & +05 & $12.9 \pm 0.1$ & $18.239 \pm 0.035$ & $15.9 \pm 1.5$ & & $-1.20 \pm 0.04$ & $-0.75 \pm 0.06$ \\
\hline$\beta \mathrm{Gem}$ & 192 & +23 & $10.34 \pm 0.09$ & $18.261 \pm 0.037$ & $14.8 \pm 2.2$ & & $-1.29 \pm 0.05$ & \\
\hline$\alpha \operatorname{Tri}$ & 139 & -31 & $19.7 \pm 0.3$ & $18.327 \pm 0.035$ & $13.3 \pm 2.0$ & & $-1.13 \pm 0.20$ & \\
\hline$\beta$ Cet & 111 & -81 & $29.4 \pm 0.7$ & $18.36 \pm 0.05$ & $21.9_{-6.8}^{+5.2}$ & & & $+0.30 \pm 0.41$ \\
\hline$\lambda$ And & 110 & -15 & $25.8 \pm 0.5$ & $18.45 \pm 0.075$ & $17 \pm 5$ & & & \\
\hline Feige 24 & 166 & -50 & $74 \pm 20$ & $18.47 \pm 0.03$ & $13 \pm 5$ & & & $-0.44 \pm 0.08$ \\
\hline WD 0621-376 & 245 & -21 & $78 \pm 23$ & $18.70 \pm 0.15$ & $14.1 \pm 6.0$ & & $-1.21 \pm 0.20$ & \\
\hline WD 2211-495 & 346 & -53 & $53 \pm 16$ & $18.76 \pm 0.15$ & $15.1 \pm 6.5$ & & $-0.96 \pm 0.16$ & $-0.27 \pm 0.16$ \\
\hline WD $1634-573$ & 330 & -07 & $37 \pm 3$ & $18.85 \pm 0.06$ & $15.8 \pm 2.5$ & & $-1.22 \pm 0.09$ & $-0.60_{-0.18}^{+0.14}$ \\
\hline$\alpha$ Vir & 316 & +51 & $80 \pm 6$ & $19.0 \pm 0.1$ & $15.8_{-5.8}^{+10.1}$ & & $-1.29_{-0.12}^{+0.10}$ & $-0.35_{-0.12}^{+0.18}$ \\
\hline GD 246 & 87 & -45 & $79 \pm 24$ & $19.11 \pm 0.025$ & $15.1 \pm 1.9$ & & $-1.26 \pm 0.06$ & $-0.55_{-0.04}^{+0.06}$ \\
\hline$\lambda \mathrm{Sco}^{\mathrm{d}}$ & 352 & -02 & $216 \pm 42$ & $19.23 \pm 0.03$ & $7.6_{-1.4}^{+1.8}$ & & $-1.64 \pm 0.05$ & $-1.32 \pm 0.05$ \\
\hline$\beta$ Cen & 312 & +01 & $161 \pm 15$ & $19.63 \pm 0.10$ & $11.7 \pm 7.5$ & $9.5_{-25.9}^{+11.9}$ & $-1.16 \pm 0.11$ & $-0.55 \pm 0.10$ \\
\hline$\gamma^{2}$ Vel & 263 & -08 & $258 \pm 35$ & $19.710 \pm 0.026$ & $21.8 \pm 2.1$ & $\begin{array}{c}-25.9 \\
24.7 \pm 3.2\end{array}$ & $-1.21 \pm 0.11$ & $-0.19 \pm 0.05$ \\
\hline$\alpha \mathrm{Cru}$ & 300 & +00 & $98 \pm 6$ & $19.85_{-0.10}^{+0.07}$ & $12.6_{-2.7}^{+3.6}$ & $11.7_{-3.4}^{+4.7}$ & $-1.30_{-0.12}^{+0.14}$ & \\
\hline $\mathrm{BD}+28^{\circ} 4211$ & 82 & -19 & $104 \pm 18$ & $19.846 \pm 0.018$ & $13.9 \pm 1.0$ & $13.4 \pm 1.3$ & $-1.20 \pm 0.10$ & \\
\hline Lan 23 & 108 & -01 & $122 \pm 37$ & $19.89_{-0.04}^{+0.25}$ & $21.9_{-17.4}^{+4.1}$ & $23.5_{-23.4}^{+5.3}$ & $-1.31_{-0.25}^{+0.07}$ & \\
\hline$\mu \mathrm{Col}^{\mathrm{e}}$ & 237 & -27 & $400_{-70}^{+100}$ & $19.86 \pm 0.015$ & $6.9_{-1.8}^{+6.9}$ & $4.5_{-3.1}^{+8.2}$ & $-1.18 \pm 0.02$ & $-0.27 \pm 0.02$ \\
\hline$\zeta$ Pup & 256 & -05 & $429 \pm 94$ & $19.963 \pm 0.026$ & $14.0 \pm 2.3$ & $13.7_{-2.8}^{+2.7}$ & $-1.28_{-0.07}^{+0.17}$ & $-0.40_{-0.03}^{+0.07}$ \\
\hline TD1 32709 & 233 & +28 & $520 \pm 90$ & $20.03 \pm 0.10$ & $18.6 \pm 5.3$ & $19.1_{-6.4}^{+6.8}$ & $-1.53 \pm 0.14$ & \\
\hline WD $1034+001$ & 248 & +48 & $155_{-43}^{+58}$ & $20.07 \pm 0.07$ & $21.4 \pm 5.3$ & $22.3 \pm 6.3$ & $-1.42 \pm 0.12$ & \\
\hline
\end{tabular}


Table 3-Continued

\begin{tabular}{|c|c|c|c|c|c|c|c|c|}
\hline Target & $\begin{array}{c}1 \\
(\mathrm{deg})\end{array}$ & $\begin{array}{c}\mathrm{b} \\
(\mathrm{deg})\end{array}$ & $\begin{array}{c}\mathrm{d} \\
(\mathrm{pc})\end{array}$ & $\log N(\mathrm{H} \mathrm{I})^{\mathrm{a}}$ & $\begin{array}{l}(\mathrm{D} / \mathrm{H})_{\operatorname{gas}^{\mathrm{a}}} \\
(\mathrm{ppm})\end{array}$ & $\begin{array}{l}(\mathrm{D} / \mathrm{H})_{\mathrm{gas}-\mathrm{LB}^{\mathrm{a}}} \\
\quad(\mathrm{ppm})\end{array}$ & $\mathrm{D}(\mathrm{Fe})^{\mathrm{a}}$ & $\mathrm{D}(\mathrm{Si})^{\mathrm{a}}$ \\
\hline $\mathrm{BD}+39^{\circ} 3226$ & 65 & +29 & $290_{-70}^{+140}$ & $20.08 \pm 0.09$ & $11.7 \pm 3.1$ & $11.2 \pm 3.4$ & $-1.38 \pm 0.11$ & $-0.79 \pm 0.21$ \\
\hline Feige 110 & 74 & -59 & $179_{-67}^{+265}$ & $20.14_{-0.10}^{+0.065}$ & $21.4_{-3.8}^{+5.7}$ & $22.1_{-44}^{+6.9}$ & & \\
\hline$\gamma$ Cas & 124 & -02 & $188 \pm 20$ & $20.16_{-0.10}^{+0.108}$ & $9.8_{-2.3}^{+2.7}$ & $\begin{array}{c}9.1_{-2.5}^{+2.9} \\
\end{array}$ & $-1.53_{-0.18}^{+0.14}$ & \\
\hline$\iota$ Ori & 210 & -20 & $407 \pm 127$ & $20.15_{-0.07}^{+0.106}$ & $14.1 \pm 2.8$ & $\begin{array}{c}-2.5 \\
13.9 \pm 3.1\end{array}$ & $-1.41_{-0.16}^{+0.21}$ & $-0.51 \pm 0.07$ \\
\hline$\delta$ Ori & 204 & -18 & $281 \pm 65$ & $20.19 \pm 0.03$ & $7.4_{-0.9}^{+1.2}$ & $6.5_{-1.0}^{+1.3}$ & $-1.56 \pm 0.04$ & \\
\hline$\theta \operatorname{Car}^{\mathrm{f}}$ & 290 & -05 & $135 \pm 9$ & $20.28 \pm 0.10$ & $\begin{array}{r}5.0 .9 \\
5.3 .4\end{array}$ & $4.0_{-4.5}^{+3.0}$ & $-1.54 \pm 0.11$ & $-1.41_{-0.12}^{+0.26}$ \\
\hline$\epsilon$ Ori & 205 & -17 & $412 \pm 154$ & $20.45_{-0.10}^{+0.08}$ & $6.3_{-1.5}^{+1.4}$ & $5.8_{-1.5}^{+1.8}$ & $-1.70_{-0.12}^{+0.14}$ & $-0.94_{-0.14}^{+0.12}$ \\
\hline PG $0038+199^{g}$ & 120 & -43 & $297_{-104}^{+164}$ & $20.48 \pm 0.04$ & $\begin{array}{c}-1.5 \\
19.1 \pm 2.6\end{array}$ & $\begin{array}{c}-1.5 \\
19.2 \pm 2.8\end{array}$ & $\begin{array}{r}-0.12 \\
-1.51_{-0.04}^{+0.05}\end{array}$ & \\
\hline LSE 44 & 313 & +13 & $554 \pm 66$ & $20.52_{-0.18}^{+0.10}$ & $22.4_{-6.2}^{+11.7}$ & $22.7_{-6.6}^{+12.8}$ & & \\
\hline JL 9 & 323 & -27 & $590 \pm 160$ & $20.78 \pm 0.05$ & $10.0 \pm 1.9$ & $9.8 \pm 2.0$ & $-1.54 \pm 0.10$ & \\
\hline HD 195965 & 86 & +05 & $794 \pm 200$ & $20.95 \pm 0.025$ & $8.5 \pm 1.6$ & $8.4 \pm 1.6$ & $-1.59 \pm 0.03$ & \\
\hline LSS 1274 & 277 & -05 & $580 \pm 100$ & $20.98 \pm 0.04$ & $7.6 \pm 1.9$ & $7.5 \pm 1.9$ & $-1.62 \pm 0.08$ & \\
\hline HD 191877 & 62 & -06 & $2200 \pm 550$ & $21.05 \pm 0.05$ & $7.8_{-1.5}^{+2.4}$ & $7.6_{-1.5}^{+2.5}$ & $-1.55 \pm 0.05$ & \\
\hline HD $90087^{\mathrm{g}}$ & 285 & -02 & $2740 \pm 800$ & $21.22 \pm 0.05$ & $8.7 \pm 1.7$ & $8.6 \pm 1.7$ & $-1.45 \pm 0.05$ & \\
\hline
\end{tabular}

${ }^{\text {a }}$ Quoted uncertainties assumed to be $1 \sigma$ (see text). $\mathrm{N}(\mathrm{H} \mathrm{I})$ is the most recent published value. N(D I) is not listed when not in original paper. $\mathrm{D} / \mathrm{H}, \mathrm{D}(\mathrm{Fe})$, and $\mathrm{D}(\mathrm{Si})$ are computed using the tablulated value of $\mathrm{N}(\mathrm{H} \mathrm{I})$.

${ }^{\mathrm{b}}$ Indicates which lines of sight are used to compute the gas-phase Local Bubble (LBg) and gas-phase local disk (LDg) $\mathrm{D} / \mathrm{H}$ values described in the text. INT indicates the intermediate regime, $\log \mathrm{N}(\mathrm{H} \mathrm{I})=19.2-20.7$.

${ }^{\mathrm{c}} \mathrm{D}(\mathrm{Fe})$ and $\mathrm{D}(\mathrm{Si})$ refer to velocity Component C (Dupin \& Gry 1998). N(H I) and N(D I) mostly in Component C.

$\mathrm{d}_{-32} \mathrm{~km} \mathrm{~s}^{-1}$ component only.

${ }^{\mathrm{e}} \mathrm{D}(\mathrm{Fe})$ and $\mathrm{D}(\mathrm{Si})$ refer to Component 1 (Howk et al. 1999), which is also called Component A (Shull \& York 1977).

${ }_{\mathrm{f}}^{\mathrm{A}}$ Abundances and depletions refer only to the H I component (Allen et al. 1992).

${ }^{g} \mathrm{~N}(\mathrm{H} \mathrm{I})$ includes $2 \mathrm{~N}\left(\mathrm{H}_{2}\right)$ and $(\mathrm{D} / \mathrm{H})_{\text {gas }}=[\mathrm{N}(\mathrm{D} \mathrm{I})+\mathrm{N}(\mathrm{HD})] /\left[\mathrm{N}(\mathrm{H} \mathrm{I})+2 \mathrm{~N}\left(\mathrm{H}_{2}\right)\right]$.

${ }^{\mathrm{h}}$ The Local Interstellar Cloud component only. For the $\mathrm{BC}$ component $(\mathrm{D} / \mathrm{H})_{\text {gas }}=5_{-5}^{+11} \mathrm{ppm}$. 
Table 4. ESTIMATES OF $(\mathrm{D} / \mathrm{H})_{\text {gas }} \operatorname{FROM}(\mathrm{D} / \mathrm{O})_{\text {gas }} \times(\mathrm{O} / \mathrm{H})_{\text {gas }}$

\begin{tabular}{|c|c|c|c|c|c|}
\hline Reference & $\begin{array}{l}\text { Oxygen } \\
\text { Data Source }\end{array}$ & $\begin{array}{l}\text { Number } \\
\text { of LOS }\end{array}$ & $\begin{array}{l}\text { Oxygen LOS } \\
\text { distance (pc) }\end{array}$ & $\begin{array}{l}(\mathrm{O} / \mathrm{H})_{\text {gas }} \\
(\mathrm{ppm})\end{array}$ & $\begin{array}{l}(\mathrm{D} / \mathrm{H})_{\text {gas }^{\mathrm{a}}} \\
(\mathrm{ppm})\end{array}$ \\
\hline \multicolumn{6}{|l|}{ Hébrard \& Moos (2003) } \\
\hline cite Meyer (2001) & GHRS, STIS & 29 & $150-5010$ & $343 \pm 15$ & $13.2 \pm 0.8$ \\
\hline André et al. (2003) & FUSE & 19 & $840-5010$ & $408 \pm 13$ & $15.7 \pm 0.9$ \\
\hline include Meyer et al. (1998) & GHRS & 32 & $150-5010$ & $377 \pm 10$ & $14.5 \pm 0.7$ \\
\hline include 5 dense LOS & GHRS & 37 & $150-5010$ & $362 \pm 11$ & $13.9 \pm 0.7$ \\
\hline Oliveira et al. (2005) & FUSE & 11 & $2.6-128$ & $345 \pm 19$ & $13.2 \pm 0.9$ \\
\hline Frisch \& Slavin $(2003)^{\mathrm{b}}$ & Models 2,8 & - & 3 & $380 \pm 30$ & $14.6 \pm 1.3$ \\
\hline Asplund et al. (2004) & solar ${ }^{\mathrm{c}}$ & - & - & $458 \pm 53$ & $17.6 \pm 2.1$ \\
\hline if $120 \mathrm{ppm}$ in dust & - & - & - & $338 \pm 53$ & $13.0 \pm 2.0$ \\
\hline Meléndez (2004) & solar & - & - & $390 \pm 63$ & $15.0 \pm 2.4$ \\
\hline Wood et al. (2004) & - & - & $3.2-94$ & - & $15.6 \pm 0.4$ \\
\hline
\end{tabular}

${ }^{\text {a Assuming }}(\mathrm{D} / \mathrm{O})_{\text {gas }}=(3.84 \pm 0.16) \times 10^{-2}(1 \sigma)$ (Hébrard \& Moos 2003).

${ }^{\mathrm{b}}$ Frisch \& Slavin (2003) do not assign an error to $(\mathrm{O} / \mathrm{H})_{\text {gas }}$. We arbitrarily assign an error of $8 \%$ based on the discussion in their paper.

${ }^{\mathrm{c}}$ Column 5 lists $(\mathrm{O} / \mathrm{H})_{\odot}$, the total abundance ratio in the solar photosphere.

Table 5. CORRELATION PARAMETERS

\begin{tabular}{lllrrrrrl}
\hline \hline \multicolumn{1}{c}{ Correlation $^{\mathrm{a}}$} & \multicolumn{1}{c}{$\log N(\mathrm{H} \mathrm{I})$} & $\mathrm{N}$ & $a$ & $\pm 1 \sigma$ & $b$ & $\pm 1 \sigma$ & Spearman & Significance \\
\hline$(\mathrm{D} / \mathrm{H})_{\text {gas }}$ vs. $\mathrm{D}(\mathrm{Fe})$ & all & 38 & 30.00 & 1.60 & 13.04 & 1.15 & 0.483 & $0.0021(2.9 \sigma)$ \\
$(\mathrm{D} / \mathrm{H})_{\text {gas }}$ vs. $\mathrm{D}(\mathrm{Fe})$ & HST,FUSE,IMAPS & 29 & 29.14 & 1.73 & 12.24 & 1.30 & 0.398 & $0.0324(2.1 \sigma)$ \\
$(\mathrm{D} / \mathrm{H})_{\text {gas }}$ vs. $\mathrm{D}(\mathrm{Fe})$ & $\geq 19.0$ & 24 & 35.04 & 3.54 & 16.68 & 2.37 & 0.534 & $0.0072(2.6 \sigma)$ \\
$(\mathrm{D} / \mathrm{H})_{\text {gas }}$ vs. $\mathrm{D}(\mathrm{Si})$ & all & 20 & 20.09 & 0.81 & 9.72 & 1.05 & 0.325 & $0.157(1.4 \sigma)$ \\
$(\mathrm{D} / \mathrm{H})_{\text {gas }}$ vs. $\mathrm{D}(\mathrm{Si})$ & $\geq 19.0$ & 11 & 19.27 & 0.86 & 10.79 & 0.984 & 0.664 & $0.026(2.1 \sigma)$ \\
$(\mathrm{D} / \mathrm{Fe})_{\text {gas }}$ vs $\log N(\mathrm{H} \mathrm{I})$ & all & 38 & -0.59 & 0.12 & 0.081 & 0.006 & 0.516 & $0.0009(3.1 \sigma)$ \\
$(\mathrm{D} / \mathrm{Fe})_{\text {gas }}$ vs $\log N(\mathrm{H} \mathrm{I})$ & $\geq 19.2$ & 22 & 1.31 & 0.47 & -0.011 & 0.023 & 0.0936 & $0.679(0.4 \sigma)$ \\
$(\mathrm{D} / \mathrm{H})_{\text {gas }-\mathrm{LB} \text { vs. } \log T_{01}}$ & all & 16 & -10.66 & 4.45 & 10.54 & 2.22 & 0.564 & $0.023(2.2 \sigma)$ \\
\hline
\end{tabular}

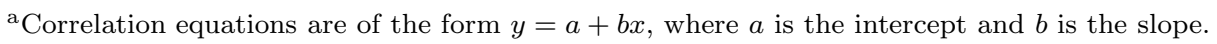


Table 6. MOLECULAR HYDROGEN TEMPERATURE MEASUREMENTS

\begin{tabular}{|c|c|c|c|c|c|c|}
\hline Target & $\log N(\mathrm{H} \mathrm{I})^{\mathrm{a}}$ & $\log N\left(\mathrm{H}_{2}\right)^{\mathrm{a}}$ & $\log f\left(\mathrm{H}_{2}\right)^{\mathrm{a}}$ & $\begin{array}{l}(\mathrm{D} / \mathrm{H})_{\mathrm{gas}-\mathrm{LB}^{\mathrm{a}}} \\
\quad(\mathrm{ppm})\end{array}$ & $\begin{array}{l}T_{01}{ }^{\mathrm{a}} \\
(\mathrm{K})\end{array}$ & Refs. \\
\hline$\gamma^{2} \mathrm{Vel}^{\mathrm{d}}$ & $19.710 \pm 0.026$ & 14.23 & -5.18 & $24.7 \pm 3.2$ & $553_{-292}^{+1150}$ & 1,2 \\
\hline $\mathrm{BD}+28^{\circ} 4211$ & $19.846 \pm 0.018$ & $15.13_{-0.08}^{+0.20}$ & $-4.43_{-0.08}^{+0.20}$ & $13.4 \pm 1.3$ & $\begin{array}{l}97_{-22}^{+16} \\
\end{array}$ & 11 \\
\hline Lan 23 & $19.89_{-0.04}^{+0.25}$ & $15.11^{-0.00}$ & $-4.48^{-0.00}$ & $23.5_{-23.4}^{+5.3}$ & $286_{-90}^{+190}$ & 4 \\
\hline$\mu \mathrm{Col}^{\mathrm{d}}$ & $19.86 \pm 0.015$ & 15.51 & -4.09 & $4.5_{-3.1}^{+8.2}$ & $\begin{array}{c}-90 \\
98_{-13}^{+16}\end{array}$ & 2,5 \\
\hline$\zeta \mathrm{Pup}^{\mathrm{d}}$ & $19.963 \pm 0.026$ & 14.45 & -5.21 & $\begin{array}{r}13.7_{-2.8}^{+2.1} \\
\end{array}$ & $382_{-158}^{+3380}$ & 1,2 \\
\hline TD1 $32709^{c}$ & $20.03 \pm 0.10$ & $14.48_{-0.11}^{+0.12}$ & $-5.25_{-0.14}^{+0.15}$ & $19.1_{-6.4}^{+6.8}$ & $\begin{array}{l}-158 \\
292 \pm 83\end{array}$ & 14 \\
\hline WD $1034+001^{\mathrm{c}}$ & $20.07 \pm 0.07$ & $15.72_{-0.12}^{+0.13}$ & $-4.05_{-0.13}^{+0.14}$ & $22.3 \pm 6.3$ & $341 \pm 75$ & 14 \\
\hline $\mathrm{BD}+39^{\circ} 3226$ & $20.08 \pm 0.09$ & $15.65_{-0.07}^{+0.06}$ & $-4.13 \pm 0.11$ & $11.2 \pm 3.4$ & $104 \pm 27$ & 14 \\
\hline$\iota \mathrm{Ori}^{\mathrm{d}}$ & $20.16_{-0.07}^{+0.06}$ & $14.69^{-0.07}$ & -5.17 & $13.6_{-30}^{+3.1}$ & $481_{-232}^{+734}$ & 2,6 \\
\hline$\delta$ Ori & $20.19 \pm 0.03$ & 14.74 & -5.16 & $6.5_{-1.0}^{+1.3}$ & $598_{-243}^{+570}$ & 7,13 \\
\hline$\theta$ Car & $20.28 \pm 0.10$ & 15.02 & -4.96 & $4.0_{-4.5}^{+\frac{1}{3} .0}$ & $\begin{array}{l}96 \pm 9 \\
-243\end{array}$ & 10 \\
\hline$\epsilon$ Ori & $20.45_{-0.10}^{+0.08}$ & 16.28 & -3.89 & $5.8_{-1.5}^{+1.8}$ & $89_{-24}^{+40}$ & 6,13 \\
\hline PG $0038+199^{b}$ & $20.48 \pm 0.04$ & $19.33 \pm 0.02$ & $-0.85 \pm 0.05$ & $19.2 \pm 2.8$ & $139 \pm 9$ & 8 \\
\hline JL $9^{\mathrm{b}}$ & $20.81 \pm 0.05$ & $19.25 \pm 0.015$ & $-1.26 \pm 0.06$ & $9.3 \pm 2.0$ & $89 \pm 3$ & 9 \\
\hline LSS $1274^{\mathrm{b}}$ & $20.99 \pm 0.04$ & $19.10 \pm 0.02$ & $-1.59 \pm 0.05$ & $7.3 \pm 1.9$ & $64 \pm 2$ & 9 \\
\hline HD $90087^{\mathrm{b}}$ & $21.22 \pm 0.05$ & $19.92 \pm 0.02$ & $-1.00 \pm 0.05$ & $8.6 \pm 1.7$ & $79 \pm 2$ & 12 \\
\hline
\end{tabular}

${ }^{\text {a }}$ Quoted uncertainties are $1 \sigma \cdot f\left(\mathrm{H}_{2}\right)=2 N\left(\mathrm{H}_{2}\right) /\left[2 N\left(\mathrm{H}_{2}\right)+N(\mathrm{H} \mathrm{I})\right]$.

$T_{01}=171 / \ln [9 N(\mathrm{~J}=0) / N(\mathrm{~J}=1)] \mathrm{K}$.

${ }^{\mathrm{b}} N(\mathrm{H} \mathrm{I})$ includes $2 N\left(\mathrm{H}_{2}\right)$ and $(\mathrm{D} / \mathrm{H})_{\text {gas }-\mathrm{LB}}=[N(\mathrm{D} \mathrm{I})+N(\mathrm{HD})] /\left[N(\mathrm{H} \mathrm{I})+2 N\left(\mathrm{H}_{2}\right)\right]$.

${ }^{\mathrm{c}} T_{02}$ is given instead of $T_{01}$ which is indeterminate.

${ }^{\mathrm{d}}$ Errors in $N(\mathrm{~J}=0)$ and $N(\mathrm{~J}=1)$ are assumed to be $0.08(1 \sigma)$.

References. - (1) Sonneborn et al. 2000. (2) Savage et al. 1977. (3) Bluhm et al. 1999. (4) Oliveira et al. 2003. (5) York \& Rogerson 1976. (6) Laurent et al. 1979. (7) Jenkins et al. 1999. (8) Williger et al. 2005. (9) Wood et al. 2004. (10) Allen et al. 1992. (11) Sonneborn et al. 2002. (12) Hébrard et al. 2005. (13) Jenkins et al. 2000b. (14) Oliveira et al. 2006. 


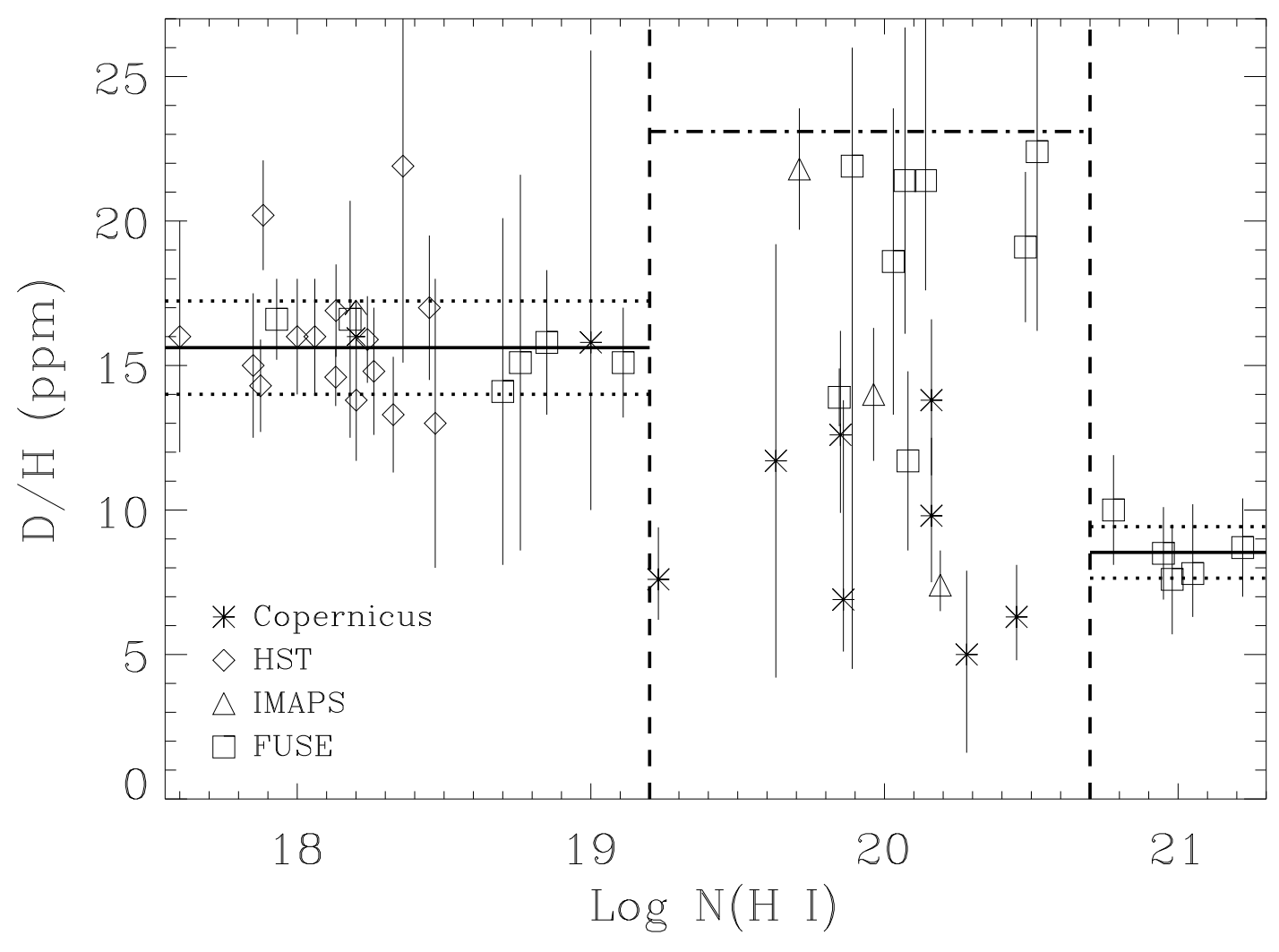

Fig. 1.- Plot of the gas-phase D/H values (not corrected for the Local Bubble foreground) vs. the hydrogen column density for the 47 lines of sight listed in Table 3 . The symbols for each data point indicate the spacecraft that observed the line of sight. Error bars are $\pm 1 \sigma$. Vertical dashed lines indicate lines of sight inside the Local Bubble $[\log N(\mathrm{H} \mathrm{I})<19.2$ $\left.\mathrm{cm}^{-2}\right]$ and the intermediate region $[\log N(\mathrm{H} \mathrm{I})$ between 19.2 and 20.7]. The solid horizontal lines indicate the mean values of $(\mathrm{D} / \mathrm{H})_{\text {gas }}$ for the low and high $N(\mathrm{H} \mathrm{I})$ regions, and the dotted horizontal lines indicate the $\pm 1 \sigma$ about the mean value. The dash-dot horizontal line is the mean value of $(\mathrm{D} / \mathrm{H})_{\text {gas }-\mathrm{LB}}$ for the highest five points in the intermediate region $\left(\gamma^{2}\right.$ Vel, Lan 23, WD 1034+001, Feige 110, and LSE 44) after subtracting the foreground Local Bubble contributions to the hydrogen and deuterium column densities along the lines of sight. See discussion in $\S 7$. 


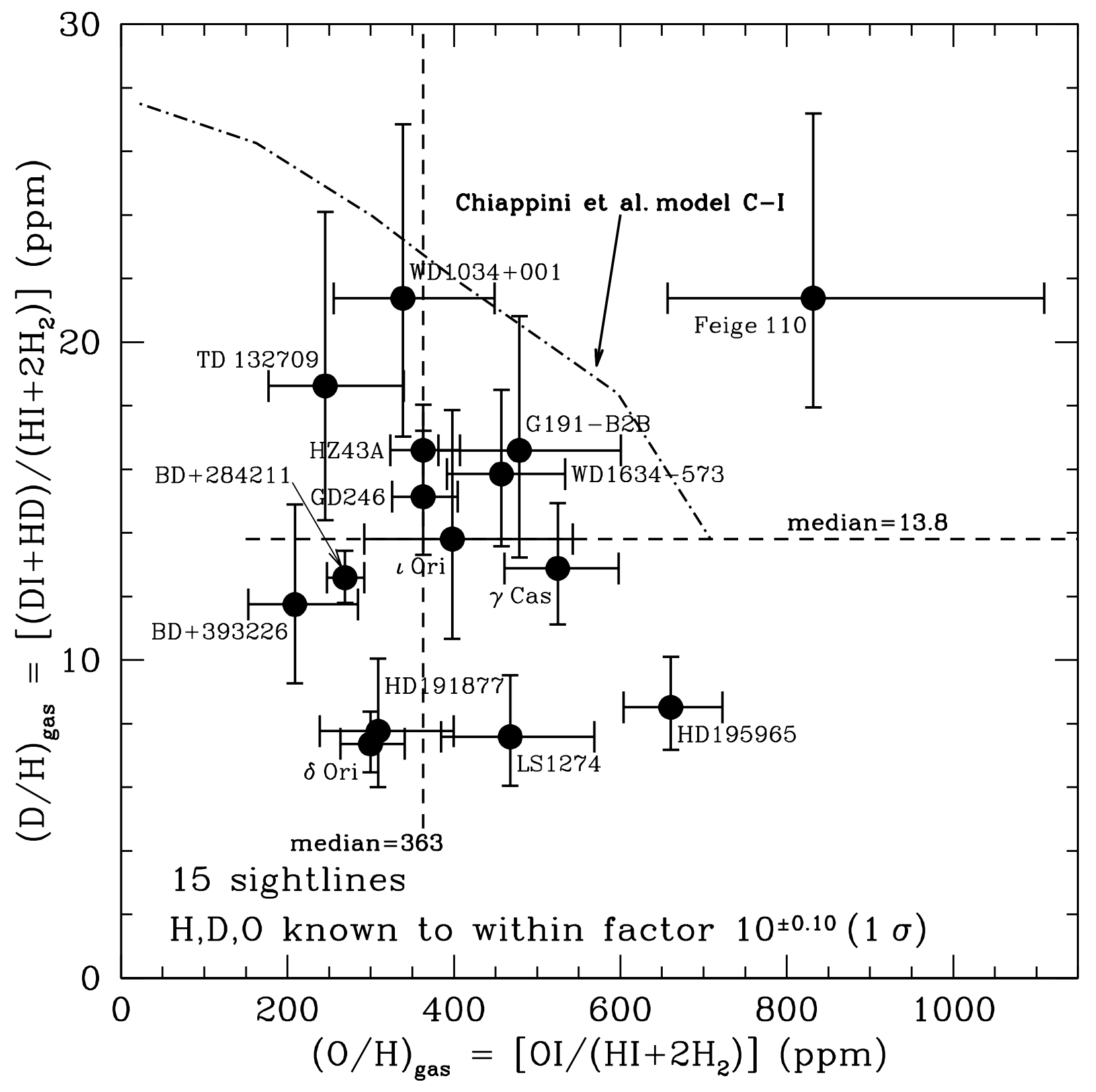

Fig. 2.- $(\mathrm{D} / \mathrm{H})_{\text {gas }}$ vs. $(\mathrm{O} / \mathrm{H})_{\text {gas }}$ with $1 \sigma$ errors (see text). The data do not show anticorrelation of $(\mathrm{D} / \mathrm{H})$ with $(\mathrm{O} / \mathrm{H})$, as would result from variable astration or infall, such as model C-I from Chiappini et al. (2002). The full range of $\mathrm{D} / \mathrm{H}$ is seen for sightlines with $(\mathrm{O} / \mathrm{H})_{\text {gas }}$ between 300 and $390 \mathrm{ppm}$. Depletion of $\mathrm{D}$ into interstellar dust appears to be the most viable explanation for the observed variations in $(\mathrm{D} / \mathrm{H})_{\text {gas }}$.

Sightlines: WD 1634-573 (Wood et al. 2002); GD 246 (Oliveira et al. 2003); BD+284211 (Sonneborn et al. 2002; Spitzer et al. 1974); Feige 110 (Friedmann et al. 2002; Hébrard et al. 2005); $\gamma$ Cas (Ferlet et al. 1980; Meyer et al. 1988; Meyer 2001); $\delta$ Ori (Jenkins et al. 1999; Meyer et al. 1998; Meyer 2001); $\iota$ Ori (Laurent et al. 1979; Meyer et al. 1998; Meyer 2001); HD 195965 and HD 191877 (Hoopes et al. 2003); HZ 43A: (Kruk et al. 2002); G 191-B2B (Lemoine et al. 2002); LSS 1274 (Wood et al. 2004); Hébrard \& Moos (2003). 


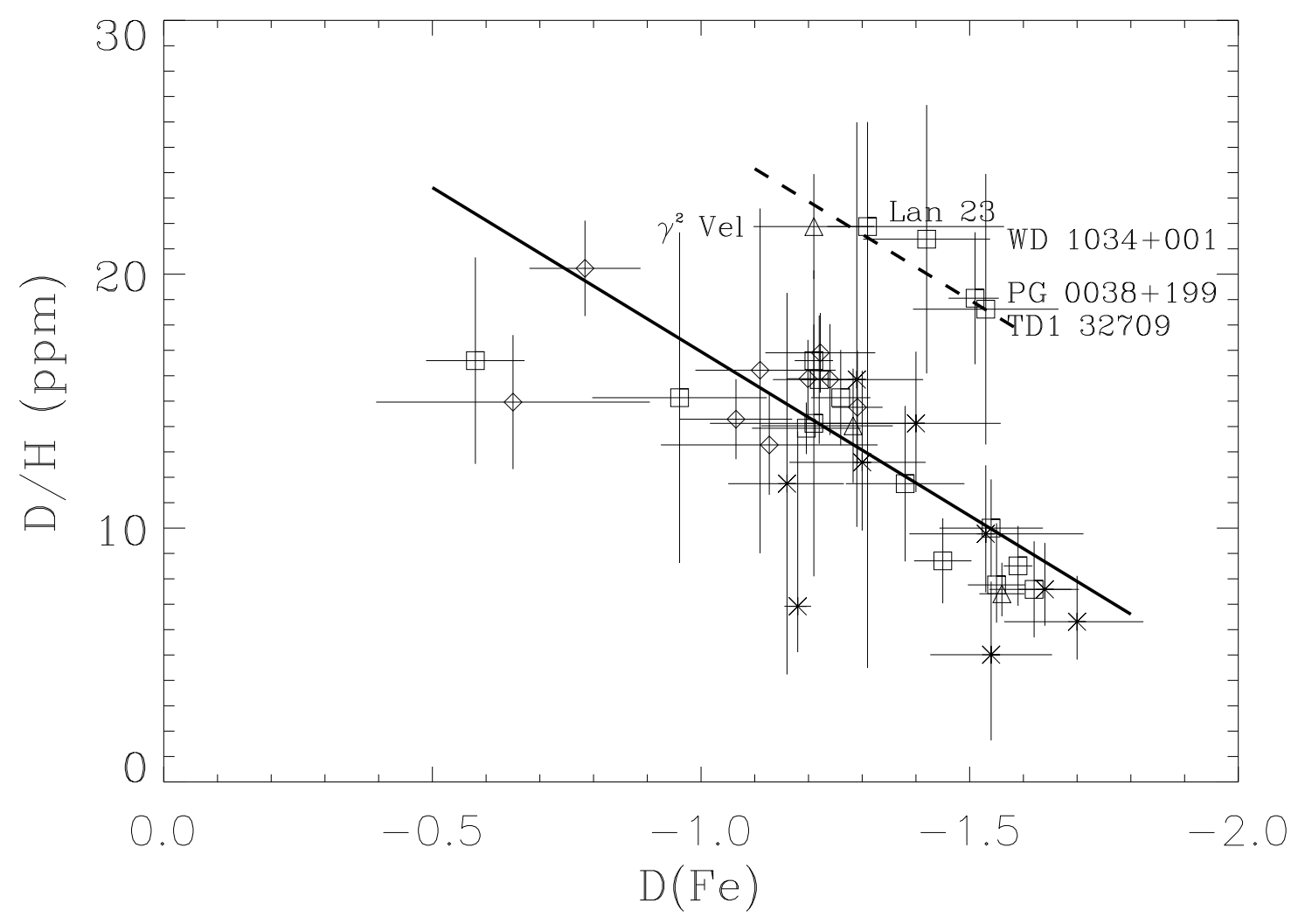

Fig. 3.- Plot of gas-phase $\mathrm{D} / \mathrm{H}$ values toward stars vs. the depletion of iron for the 38 lines of sight listed in Table 3 (ignoring upper limits). The symbols are the same as in Figure 1, and the error bars are $\pm 1 \sigma$. The solid line is the least-squares linear fit to the data weighted by the inverse errors. See $\S 6$ for a discussion of the error analysis technique. Five lines of sight with $(\mathrm{D} / \mathrm{H})_{\text {gas }}$ well above the linear fit are identified. The dashed line has the same slope as the solid line but is displaced upward by $8.5 \mathrm{ppm}$. See discussion in $\S 6.3 .4$. 


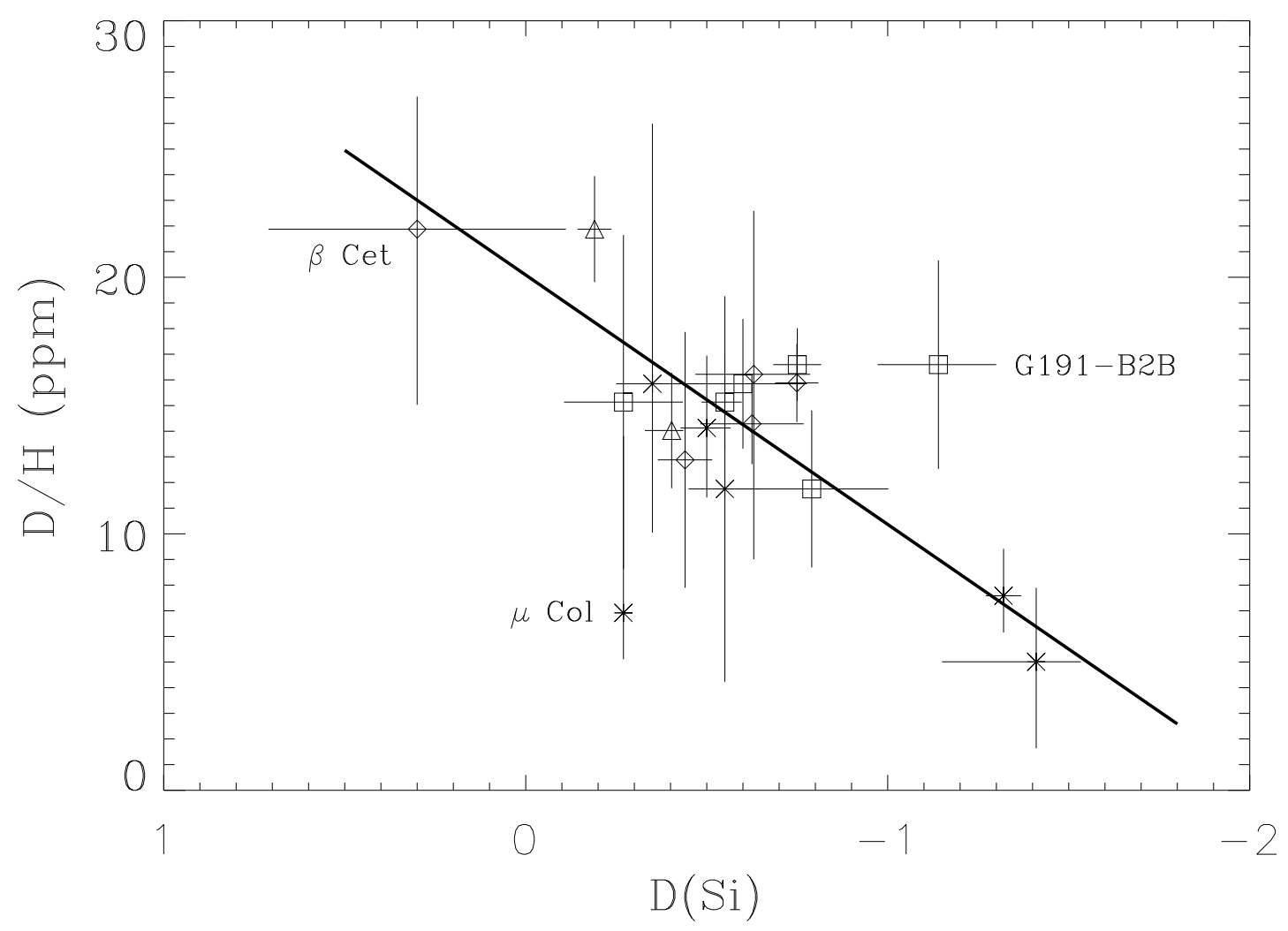

Fig. 4.- Plot of gas-phase $\mathrm{D} / \mathrm{H}$ values toward stars vs. the depletion of silicon for the 20 lines of sight (ignoring upper limits) listed in Table 3. The symbols are the same as in Figure 1, and the error bars are $\pm 1 \sigma$. The solid line is the least-squares linear fit to the data (except for upper limits) weighted by the inverse errors. Lines of sight to three stars are identified. 


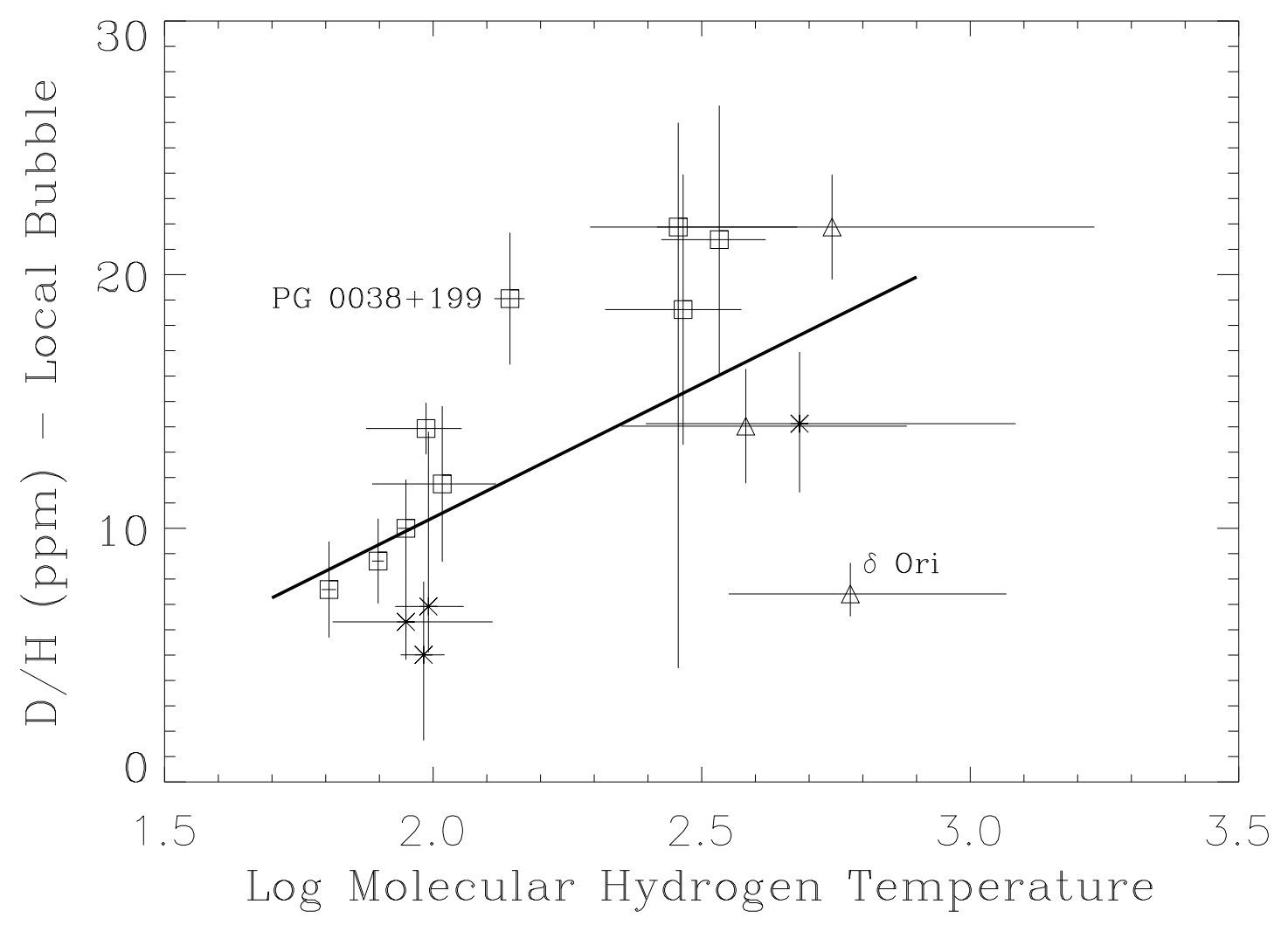

Fig. 5.- Plot of gas-phase D/H values toward stars (after correction for the Local Bubble foreground) vs. $T_{01}$, the excitation temperature of molecular hydrogen between the $\mathrm{J}=0$ and $\mathrm{J}=1$ rotational levels. The solid line is the least-squares linear fit to the 16 data points (see Table 6) weighted by the inverse errors. Two outliers are identified. 


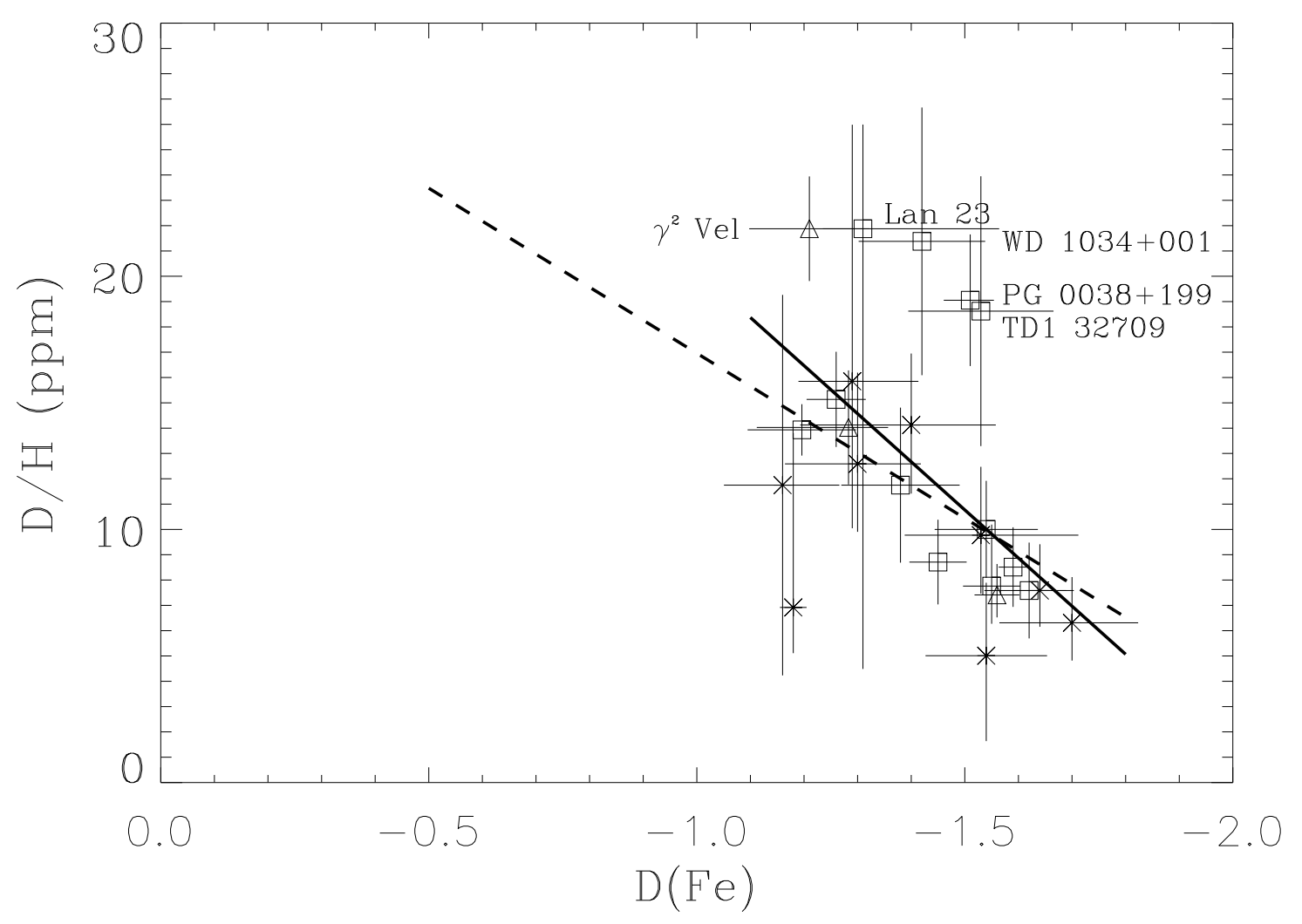

Fig. 6.- Plot of gas-phase $\mathrm{D} / \mathrm{H}$ values toward stars vs. the depletion of iron for the 24 lines of sight listed in Table 3 with $\log N(\mathrm{H} \mathrm{I}) \geq 19.0$. The symbols are the same as in Figure 1, and the error bars are $\pm 1 \sigma$. The solid line is the least-squares linear fit to the data weighted by the inverse errors. The dashed line is the fit to the 38 lines of sight covering the full range of $\log N(\mathrm{H} \mathrm{I})$ as shown in Figure 3. 


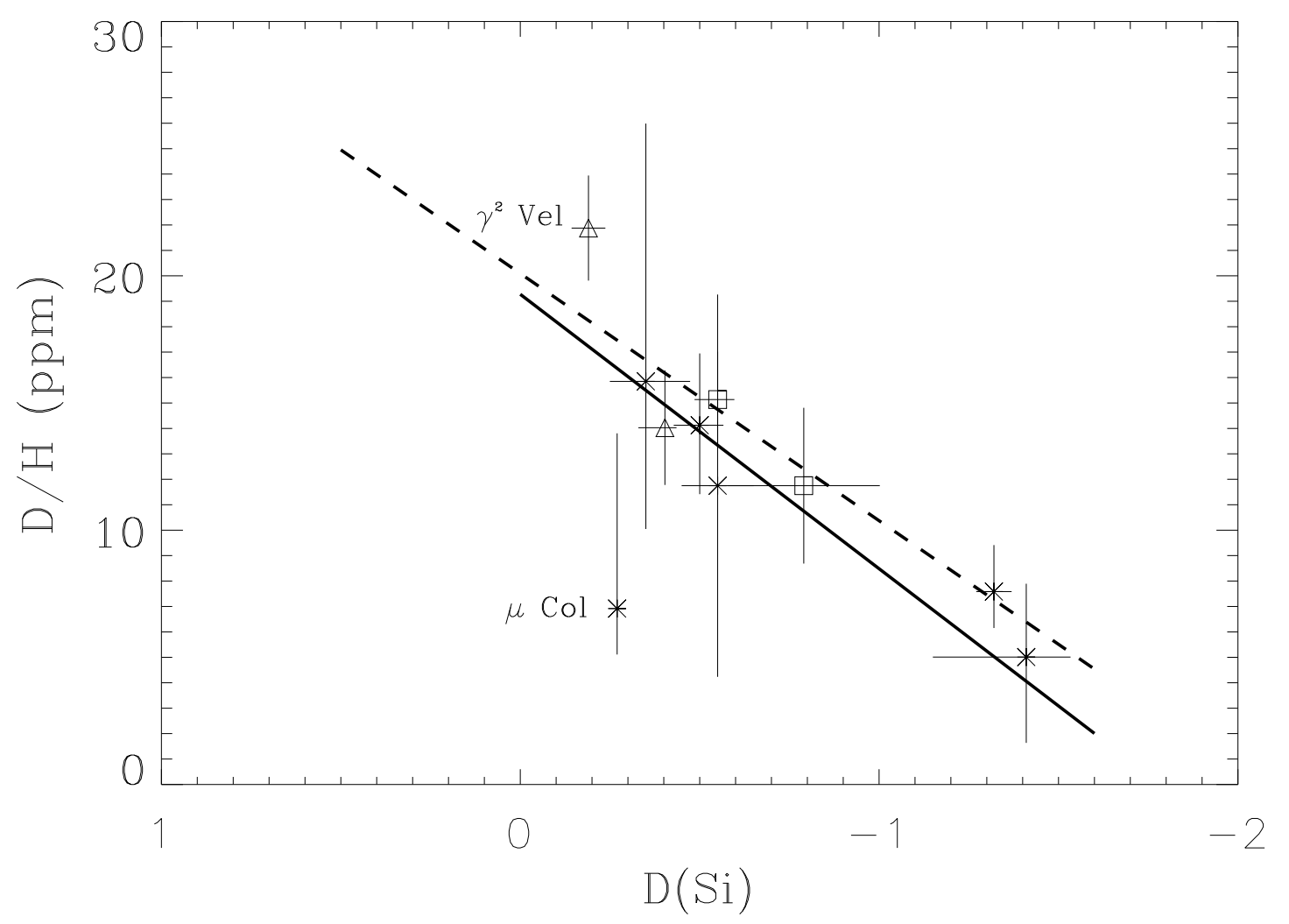

Fig. 7.- Plot of gas-phase $\mathrm{D} / \mathrm{H}$ values toward stars vs. the depletion of silicon for the 11 lines of sight listed in Table 3 with $\log N(\mathrm{H} \mathrm{I}) \geq 19.0$. The symbols are the same as in Figure 1, and the error bars are $\pm 1 \sigma$. The solid line is the least-squares linear fit to the data weighted by the inverse errors. The dashed line is the fit to the 20 lines of sight covering the full range of $\log N(\mathrm{H} \mathrm{I})$ as shown in Figure 4. 


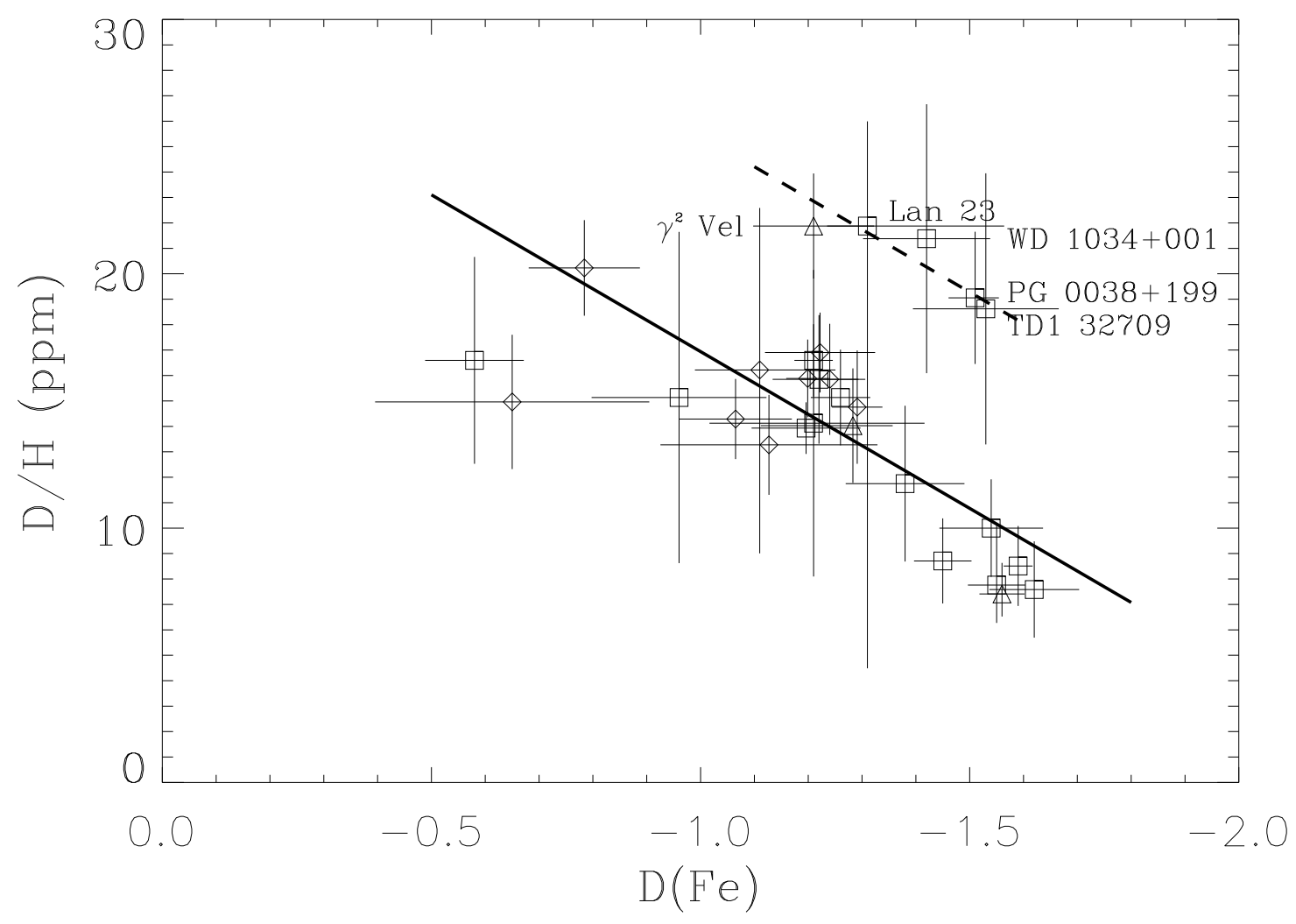

Fig. 8. - Same as Figure 3 except using data only from STIS, GHRS, IMAPS, and FUSE. 


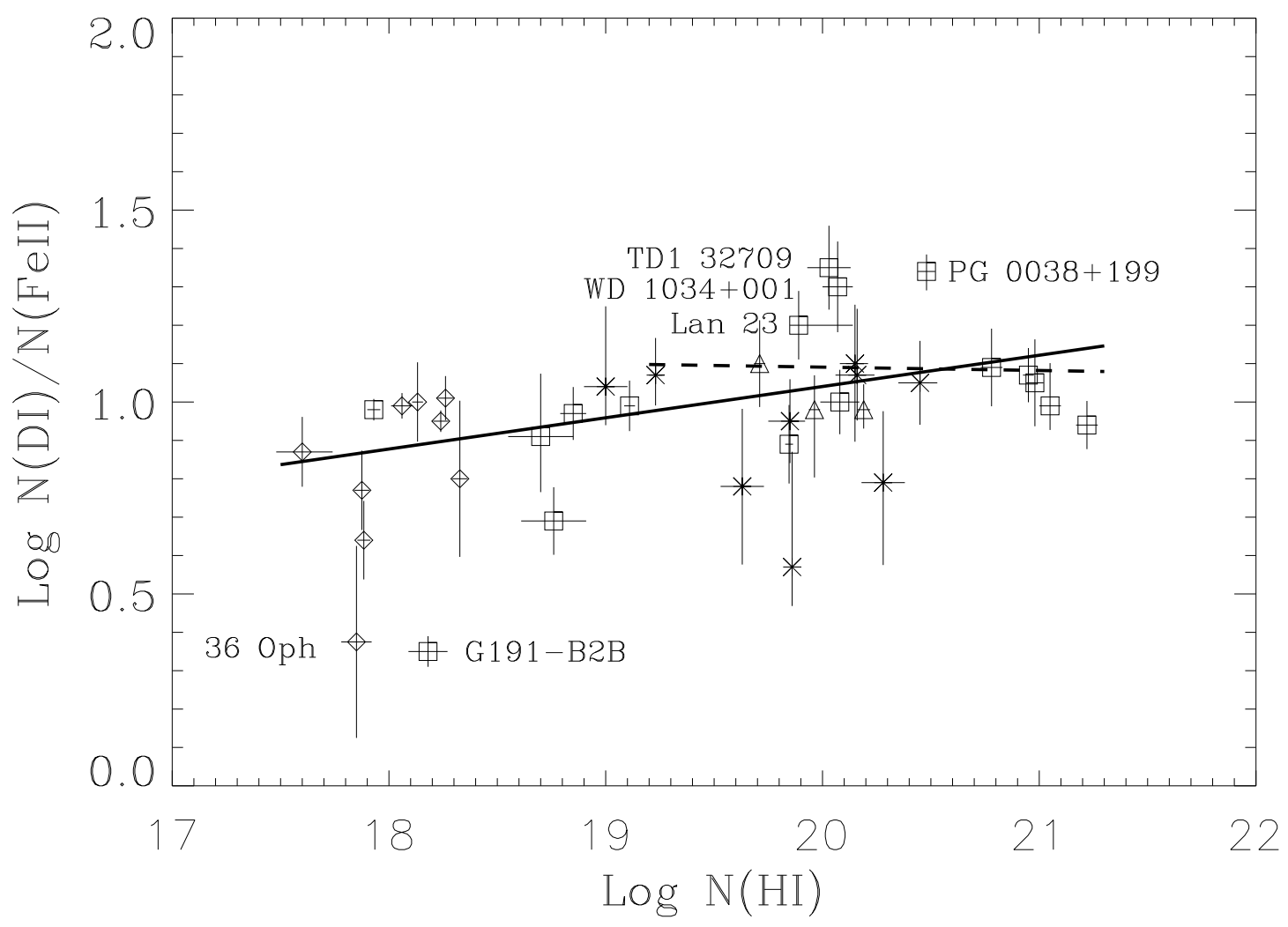

Fig. 9.- Column density ratios $\log [N(\mathrm{D}$ I $) / N(\mathrm{Fe}$ II $)]$ vs. $\log N(\mathrm{H}$ I) for all lines of sight with symbol coding the same as in Figure 1. The solid line is a weighted least-squares fit to all of the data points, and the dashed line is a similar fit to only the data beyond the Local Bubble $(\log N(\mathrm{H} \mathrm{I})>19.2)$. 